Gibert y Oliver, Agustí María

Fauna ictiológica de Catalunya 



\title{
FAUNA ICTIOLÓGICA
}

$=$ DE CATALUNYA $=$

no

\section{AGUSTI M.^ GIBERT}

div!dus correspenent de la Reyal Academia de Ciencies y Arts de Barcelona; Soct hunorari dé 1'Institucí́ Catalana d'Historia Natural, etc.

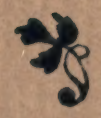

\author{
BARCELONA \\ Imp. do J. Bartra Labordo \\ Sarta Agna, núm. 20 \\ 1813
}



FAUNA ICTIOLÓGICA DE CATALUNYA 

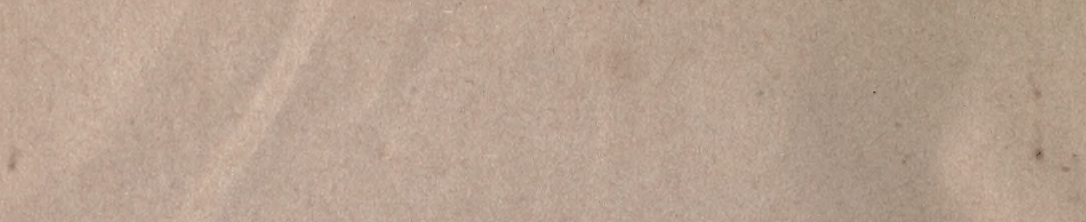

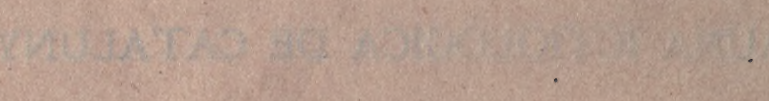




\title{
FAUNA ICTIOLÓGICA \\ $=$ DE CATALUNYA =
}

Catálech rahonat dels peixos observats en el litoral y en les aygues dolçes catalanes

\section{PER - \\ AGUSTI M.^ GIBERT}

Individus corresponent de la Reyal Academia de Ciencies y Arts de Barcelona; Soci honorari de l'Institució Catalana d'Historia Natural, etc.

\author{
BARCELONA \\ Imp. de J. Bartra Laborde \\ Santa Agna, nủm. 20 \\ 1913
}




\section{ADIDO TOITDI AVUA}

\section{AVIUIATAD JC}

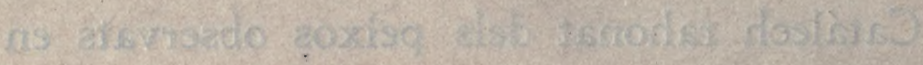

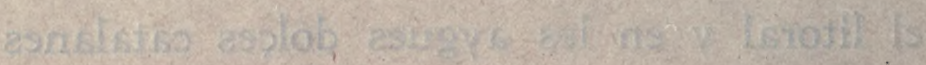
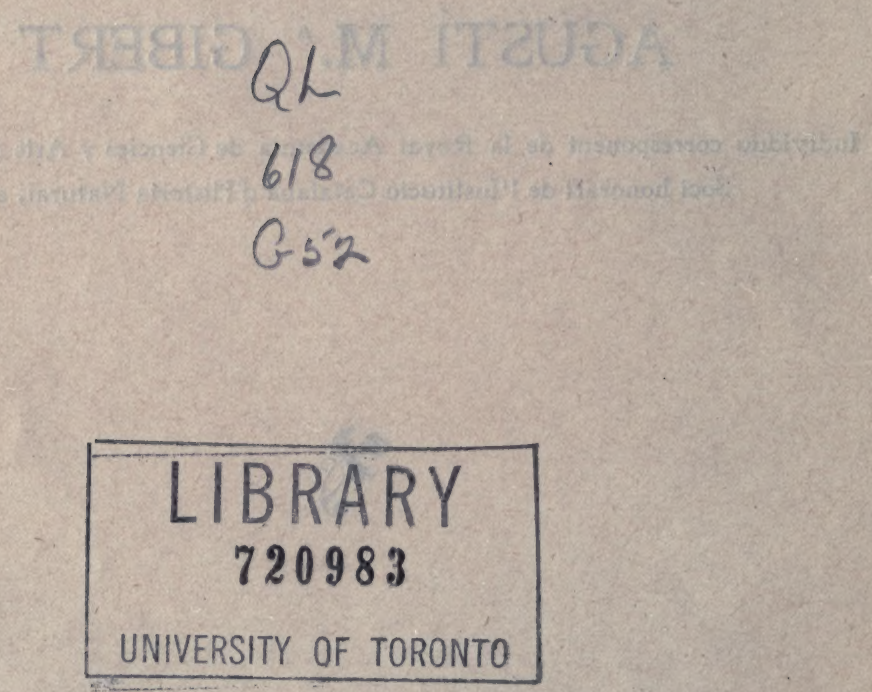


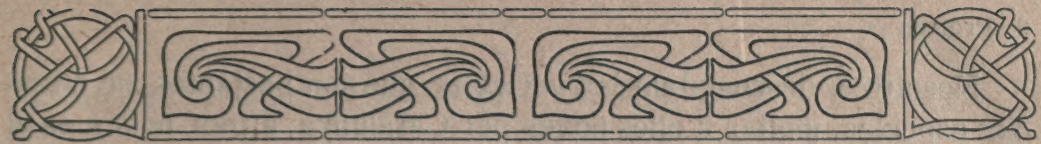

\section{PRÓLECH}

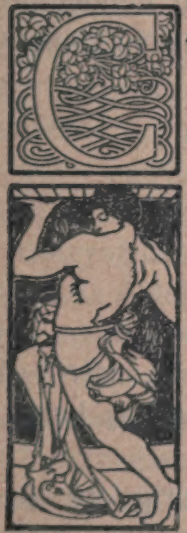

AL dirho. L'estat de nostra Ictiología, una de les parts mes descuidada de la Fauna Catalana, fa ja alguns anys ens hi feu fixar les mirades y'ns mogué á empendrer amb mes bona voluntat que confiança en les nostres escasses forçes, $1^{\prime}$ estudi d'aquesta importantíssima branca de la Zoología, amb el propósit de publicar amb temps y paciencia'l fruyt cullidor de nostres llargues y penoses investigacions, treball el mes complert possible sobre 'Is peixos de nostra aymada Patria que s'extén avuy desde'1 Cap Cervera fins al ríu Cenia, y de la costa al Noguera Ribagorzana vers l'interior. Empresa sembrada d'esculls que vé á esvair moltíssimes confusions y arrodoneix passats esforços.

En 1853, en Marian Graells citava 98 especies (Anales de la Academia dc Ciencias de Madrid); en Ferran Weiler en 1854, ne nombra 90 (Topografía físico-médica de las Baleares, Palma); en Francesch Barceló comprén en _el seu catálech aparegut á Madrid en 1868, 247 especies habitantes en les clássiques costes de les Mallorques y ordenades segons la classificació del Princep de Canino y Musignano, Carles Bonaparte; en Laureá Pérez Arcas durant el seu pas per nostra Universitat ne coleccioná y catalogá 147: en Antoni Sánchez Comendador afegí á l'anterior Catálech altres especies que adquirí y estudiá, sumant en conjunt 190 especies y darrerament en Jaume Ferrer y Aledo en el Catálech dels peixos de l'illa de Menorca publicat en 1906, n' hi enclou, amb les varietats, unes 224. 
Nosaltres, descomptant les varietats, que son bastantes, ne citem 322 especies, moltes delles noves per Catalunya, altres noves en el Mediterrá, una de nova per Europa, y poques, molt poques per classificar.

Pera fixar bé y amb seguretat les especies, hi acompanyem la complexa sinonimia científica de totes elles, car es molt comú veurer especies repetides amb distinta denominació; y á continuació del nom de género y especie hi posem els diferents noms vulgars tan comarcals com de localitat, referencies de gran utilitat en els casos de difícil ó duptosa classificació que fan planer el camí y treuhen 1'espessa brolla que l'amaga.

No son tan prchs, com se diu, els noms vulgars del conjunt de peixos coneguts, Lo que hi ha es que en les transaccions mercantívoles, una esperie ó conjunt d' especies científiques pren denominacions especials, "la quantitat moltes vegades fa la denominació comercial; veusen aquí nota, per exemple:

Bastina que compren tots els Selácits.

Llus.

Llenguado (y rénıols).

Mollera (y mollera brótola).

Raps.

Gatneu.

Peix blau: sardina, aladroch ó seitó, amploia ó meleta, latría ó sardina alatxa, alatxes ó sabogues, agulles, surell, surella, barat, cavalla, palomides, verderols, pampols, etc.

Raballa: molls, lluernes, refet, peona, borratxo, xuriguers, jurioles, malarmat, panegal, rata, aranyes, escorpores ó polles, pelayes, pagells, bogaravell, pagres, serrans, xucles, jerrets, boga, galls, etc.

Moixons.

Llússara ó maira.

Pelut (Part del): pelut d'escata, caps sechs ó claus, pixotes vermelles, $y^{\prime} 1$ peix petit de tota mena.

Aquestes son les denominacions especials ó mercantivoles pel peix agafat á la vaca y al bou, sens compendri'l que's torna á llençar á la mar, com son: cavalls marins, titolots, trompeters, gallets de mar, musichs, llampreses, etc.

La classificació que havém adoptat ẻs la del Dr. en E. Moreau que divideix els peixos en tres subclasses, basantla en la disposició 
de llur aparell branquial: Hiobranquis, Marsipobranquis y Faringobranquis.

Aixís, donchs, els géneros y especies que composen aquest Catálech, son:

I. Hiobranquis

1I. Marsipobranquis

III. Faringobranquis

Total
Géneros 139 Especies 318

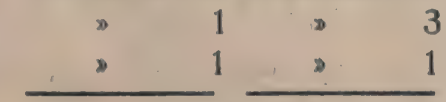

Géneros 141 Especies 322

Entre aquestes especies hi ha l'nostre Cerna (Epinephelus) Cathalonica, nova per Europa; diferentes especies no assenyalades com habitantes en el Mediterrá: Selache maximus, Cuv.; Nerophis lumbriciformis, Bp.; Lota molua, Bp.; Motella tricirrata, Günth.; Pleuronectes hirtus, Abildgaard; Salmo Salar, Linn., y una munió d'especies encar que del Mediterrá, no citades fins ara en les nostres aygues y que junt amb algunes d'aygua dolca's troben anotades en el cós de nostre treball.

Malgrat nostra perseverancia y esforç no pensem oferir una obra complerta ó del tot acabada. De les mateixes observacions constatades en el curs d'ella pot palesament col-legirse que han romás bastantes coses encara per esbrinar.

Dolçament satisfets de servir á la Ciencia y á la Patria, mancans, per $\{i$, expressar nostra gratitut envers nostre bon amich en Felip Ferrer y Vert de l'Institució Catalana d'Historia Natural y'ls qui amables en aquestes anyades s'han dignat enviarnos desinteressadament noticies, referencies, y exemplars d'especies rares; á tots sense distinció, moltes, moltíssimes mercés.

Tarragona, Jor d'Octubre, de 1910. 


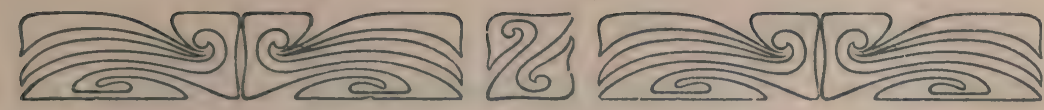

\section{= Catálech rahonat dels peixos observats} en el litoral y en les aygues dolses catalanes

Sub-classe dels Hiobranquis.

Divisió I. BRANquiocers ó Ceratobranquis.

Secció I. Plagiostoms.

Ordre I. Selacienchs (Selacha).

Sub-ordre, Squalits (Squali).

Tribu dels Squalits Hipopterienchs.

Sub-tribu dels SQUALIENCHS (SQUALII).

(Nom vulgar: Bastina).

Familia dels Scyllidide.

Genero. Scyllium, Cuv.

Scyllium Canicula, Cuv.

Synonimia: Squalus catulus, Linn., Bloch, Riss., Blainville; SquaIus canicula, Riss., J. Müller et J. Henle, C. Bonaparte, Aug. Dumeril, Barboza de Bocage et G. de Brito Capello, A. Günther, Canestrini; Galeus stellaris major, Bellon.

Nom vulgar: Gat, Barcelona, Tarragona; Gat ver, Goles de 1' Ebre; Gató, Menorca.

Scyllium catulus, Cuv.

Syn.: Scyllium stellare, CBp., Günth., Canestr.; Squalus ste. llaris, Linn., Lacep., Riss., Blainv.; Scy'llium stellaris, Riss.; Scy"- 
10 Fauna ictiológica de Catalunya

llium catulus, Müll. et Henl., A. Dumer., Bocage et Capello; Squalus caniculus, Bloch; Canicula saxatilis, Rond.; Galeus stellaris minor, Bell.

Nom vulgar: Gatvayra, Tarragona, Barcelona, Goles de l'Ebre, Menorca.

Genero, Pristiurus, Bp.

Pristiurus melanostomus, $\mathrm{CBp}$.

Syn.: Pristiurus, Artedi, Bocage et Capello; Squalus melanos tomus, Blainv.; Pristiurus melanostomus, Müll. et Henl., Agass., A. Dumer., Günth., Canestr.; Squalus Delarochianus, Blainv.; Scyllum, Artedi, Riss.; Squalus Catulus, Riss.

Nom vulgar: Muxina, Tarragona.

Especie no citada en cap Catálech de peixos de Catalunya pero molt coneguda de tots els nostres pescaires y sobre tot de la nostra pobrissalla, car el seu baix preu y abundor extraordinaria fan que molts dies á l'hivern siga'l seu plat escullit.

\section{Familia dels Alopecidae. \\ Genero, Alopias, Rafin. Alopias vulpes, Bp.}

Syn.: Alopecias vulpes, Günth.; Alopias vulpes, Müll. et Henl., A. Dumer., Bocage etCapello, Canestr.; Carcharias vulpes, Riss.; Squalus vulpes, Lacep., Riss., Blainv.; Vulpecula, Salviani; Vul. pes, Rond.; Simia, Bell.

Nom vulgar: Peix espasa per confondrel amb el Pristis antiquorum, Latham y amb el Xiphias gladius, Linn. L'únich exemplar que havem vist y del quí servem la cua fou pescat á l'art en 17 de Maig de 1911 , y pesave 10 kilograms.

Familia dels OdonTASPIDAE.

Genero. Odontaspis, Agass.

Odontaspis ferox, Agass.

Syn.: Triglochis ferox, Canestr.; Odontaspis rerox, Mull. et Henl., A. Dumer., Günth., CBp.

Nom vulgar: Salroig, Barcelona. 
Familia dels LAMNIDAE.

Genero, Lamna, Cuv.

Lamna cornubica, Cuv.

Syn.: Squalus cornubicus, Blainv., Cuv., Lacep., Riss.; Lamna cornubica, Cuv., Agass., Müll. et Henl., A. Dumer., Bocage et Capello, Günth., Canestr., CBp.

Nom vulgar: no'n té. Un sol exemplar n'havem vist pescat á l'art en 25 de Maig de 1911.

Genero, Oxyrhina, Agass.

Oxyrhina Spallanzanii, Bp.

Syn.: Lamna Spallanzanii, Günth.; Oxvrhina Spallanzani, Agass., A. Dumer., Canestr.; O.xvrhina gomphodon, Müll. et Henl., Bocage et Capello.

Nom vulgar: Solratj, Tarragona. Especie nova per Catalunya.

Genero, CARcharodon.

Carcharodon Lamia, Bp.

Syn.: Carcharodon Rondeleti, Müll. et Henl., A. Dumer., Günth., Canestr.; Carcharodon Lamia, Agass.; Carcharias lamia, Riss.; Squalus Carcharias, Riss.; Lamia, Rond.

Nom vulgar: Ca-marí, Tarragona; Marraco, Barcelona; Salproix, Menorca.

Peixos gurmants aquest y l'anterior que sostenen mortals lluytes amb el peix Emperador (Pristis antiquorum, Latham), escometen y atueixen les moles de tunyines y son ensemps objecte d'una preocupació popular, bastant extesa, car llurs peçes dentaries aconduhides en bossetes les pengen al coll dels infantons pera facilitarlos la dentició y gorirlos de la bava en les seves multiples modalitats patológiques.

Genero, Selache, Cuv.

Selache maximus, Cuv.

Syn.: Squalus elephas, Lesueur; Selache maximus, Müll. et Henl., CBp., A. Dumer., Bocage et Capel., Günth. Canestr.; Squalus maximus, Lacep., Linn., Agass. 
Nom vulgar: (Pelerin á France). Especie no citada com habitant en el Mediterrá. En Juny de 1901 se va trobar un d'aquests esqualits en les costes catalanes. (Butlletí de l'Institució Catalana d'Historia Natural, Octubre de 1907) y sabem la presencia de dos mes: l'un agafat á Savary y l'altre á Saint Tropez en les costes de Provença.

Genero, Mustelus.

Mustelus vulgaris, Müll. et Henl.

Syn.: Mustelus plebeius, CBp., Canestr.; Mustelus vulgaris, A. Dumer., Bocage et Capello, Günth.; Squalus hinnulus, Blainv.;

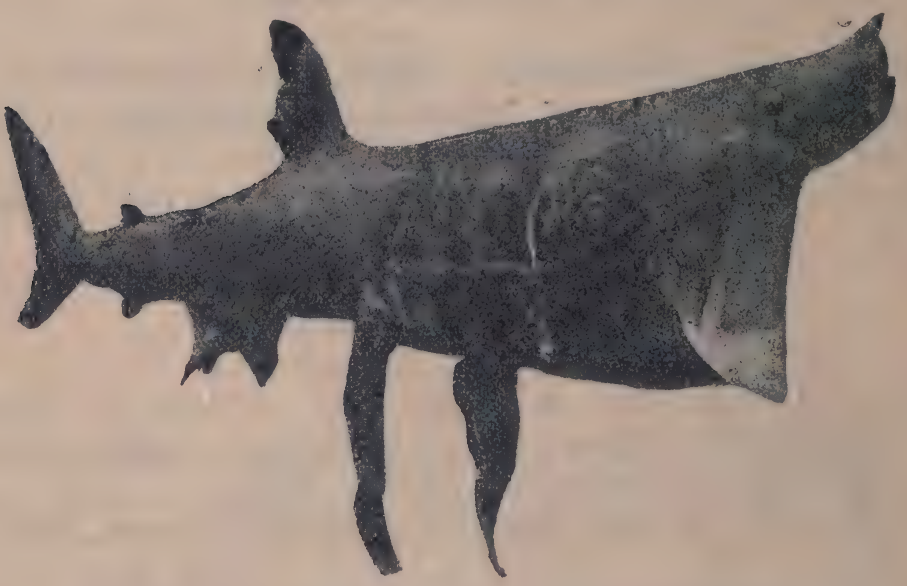

Selache maximus, Cuv.

Mustelus stellatus, Riss.; Squalus mustelns, Riss.; Galeus hinnulus, Bell.

Nom vulgar: Mussola vera, Tarragona; Mussola, Barcelona, Menorca.

Mustelus laevis, Riss.

Syn.: Mustelus equestris, CBp., Canestr.; Mustelus punctulatus, Müll. et Henl.; Mustelus laevis, Müll. et Henl., A. Dumer., Capello, Gunth., Riss.; Squalus laevis, Blainv.; Galeus laevis, Rond.

Nom vulgar: Mussola, Barcelona, Tarragona, Menorca. Especies 
menys abundantes que la Pristıurus melanostomus, CBp. (Muxina). Llur carn, particularment la de la vera, ingerida, es indigesta, produheix mareig ó una mena de borratxera acompanyada de mal de cap ó de dolor de polços (Cefalalgia suborbitaria).

\section{Familia dels Galeidae. \\ Genero, Galeus, Cuv. \\ Galetts Canis, Rond.}

Syn.: Carcharias galeus, Riss.; Squalus galeus, Linn., Bloch, Lacep., Riss., Blainv.. Cuv.: Galeus Canis, Müll. et Henl., A. Dumer., Bocag. et Capel., Günth., CBp., Canestr., Rond.; Canis galeus, Salvian.

Nom vulgar: Caçó, Tarragona; Gat, Barcelona; Camarí, Menorca.

Familia dels Zygaenidae.

Genero, Zygaena, Cuv.

Zygaena malleus, Valenc.

Syn.: Cestracion zlyaena, A. Dumer.; Sphyrna zygaena, Rafin., Müll. et Henl., Bocag. et Capel., CBp., Canestr.; Squalus zygaena, Lacep., Riss., Linn.; Z!gaena-malleus, Riss., Agass., Günth.; LIBELLA, Bell.

Nom vulgar: Llunada, Guardia civil, Tarragona, Barcelona; Llunada, Cornuda, Menorca.

\section{Familia dels Carcharide. Género, Carcharias, Cuv. Carcharias glaucus, Agass.}

Syn.: Prionodon glaucus, Bocag. et Capel., Canestr.; Carcharıas glaucus, Müll. et Henle, A. Dumer., Günth.; Squalius cœruleus, Blainv,; Squalus glaucus, Cuv., Lacep., Riss., B!ainv., Linn., Bloch, CBp.; Galeus glaucus, Duham., Rond.

Nom vulgar: Ca-marí, Tarragona; Tiburó, Menorca. Lo mateix que les dugues seguents especies, moltes vegades se venen en els mercats per peix espasa. 
Carcharias lamia, Müll. et Henl.

Syn.: Prionodon lamia, Bocag. et Capel.; Carcharias (Prionodon) lamia, A. Dumer., Günth.

Nom vulgar: Ca-marí, Tauró, Tintorera, Tarragona. Especie nova per Catalunya.

Carcharias Milberti, Val.

Syn.: Prionodon Milberti, Canestr.; Eulamía Milherti, Gill.; Squalus plumbeus, Nardó; Lamna caudata, Dekay; Carcharias coeruleus, Dekay; Carcharias Milberti, Valenc.; Prionodon, A. Dumer., Günth., Giglioli, Doderlein, Perugia.

Nom vulgar: Ca-marí, Tauró, Tintorera, Tarragona. Especie nova per Catalunya.

En 28 de Maig de 1910 vegerem un petit mascle y en 18 de Juny del mateix any en la mateixa Pescatería de Tarragona una femella molt més grossa.

Sub-tribu dels NOTIDANIENCHS.

Familia dels NOTIDANIDE.

Genero, Hexanchus, Rafin.

Hexanchus griseus, Rafin.

Syn.: Notidanus griseus, CBp., Agass., Günth., Canestr., Bocag. et Capel.; Hexanchus griseus, Müll. et Henl., A. Dumer.; Notidanus monge, Riss.; Squalus griseus, Lacep., Riss., Cuv., Blainv.

Nom vulgar; Boca dolç ó Boca dolça. Peix xovato. Especie nova per Catalunya.

Genero, HePTanchus, Müll. et Henl.

Syn.: Notidanus cinereus, CBp.; Günth.; Heptanchus cinereus, A. Dumer., Canestr.; Heptanchus cinereus, Rafin., CBP.; Squalus cinereus, Lacep. Riss. Blainv., Cuv.

Nom vulgar: Boca dolç ó Boca dolça. Peix xovato. Especie nova per Catalunya, més rara que l'anterior. 
Tribu dels SQUALITS ANHIPOPTERIENCHS.

Familia dels SPINACIDE.

Genero, Acanthias, Bp.

Acanthias vulgaris, Riss.

Syn.: Squalus acanthias, Lacep., Riss., :Blainv., Linn., Bloch' Acanthias valgaris. Müll. et Henl., CBp., A. Dumer., Günth., Bocag. et Capel., Canestr.; Mustelus svinax, Bel.

Nom vulgar: Agullat, Tarragona; Quissona, Menorca.

Acanthias Blainville, Riss.

Syn.: Acanthias Blainvillei, CBp.; Acanthias Blainvillii, Müll. et Henl., A. Dumer., Bocag. et Capel., Günth., Canestr.; Spinax Blainvillei, Agass., Riss.

Nom vulgar: Agullat xato, Tarragona; Quissona, Menorca.

Genero, SpInax, Bp. Spinax niger, $\mathrm{H}$. Cloquet.

Syn.: Acanthias svinax, Riss.; Squalus spinax, Linn.; Galeus acanthias seu spinax fuscus, Willgh.; Spinax niger, Agass., Müll. et Henl., A. Dumer, Günth., CBp., Canestr.

Nom vulgar: Negret, Negritu, Tarragona. Peix xovato.

Genero, Centrophorus, Müll. et Henl. Centrophorus granulosus, Müll. et Henl.

Syn.: Acanthorinus granulosus, Blainv.; Centrophorus granulosus, Guichenot, A. Dumer., Günth., Bocag. et Capel., Canestr.

Nom vulgar: Porch marí, Gutxu, Tarragona. Especie nova per Catalunya.

Genero, Centroscymnus, Bocag. et Capel.

Centroscymnus coelolepis, Bocag. et Capel.

Syn.: Centroscymnus coelolepis, Brit. et Capel., Vaillant; Centrophorus coelolepis; Günth.

Nom vulgar: Porch mari, Gutxu, Tarragona. Especie nova per 
Catalunya. En 25 de Juny de 1910 en la Pescatería de Tarragona n'examinarem tres exemplars pescats á palangre.

Genero, Centrina, Cuv.

Centrina vulpecula, Bel.

Syn.: Oxvnotus centrina, Rafin., A. Dumer.; Centrina Salviani, Riss., Müll. et Henl., CBp., Bocag. et Capel., Günth., Canestr.; Squalus centrina, Lacep., Riss., Blainv., Linn., Bloch; Centrina, Salvian., Rond,; Vulpecula, Bel.

Nom vulgar: Porch marí, Barcelona, Menorca; Truja, Tarragona, Torredembarra. Peix xovato. Del fetje en moltes localitats se' $n$ acostuma fer oli.

Familia dels Scymnidae.

Genero, Scymnus.

Scymnus lichia, Müll. et Henl.

Syn.: Squalus nicaensis, Riss.; Squalus americanus, Linn., Lacep., Blainv.; Scymnus nicaeensis, Riss.; Scymnus, Cuv.; Scymnus lichia, Agass., A. Dumer., Bocag. et Capel., Günth., CBp., Canestr.; Lichia, Brousson, Duham.

Nom vulgar: Negret, Negritu, Tarragona. Especie nova per Catalunya.

Familia dels SQUATINIDAE.

Genero, Squatina ó RHina.

Squatina angelus, Riss.

Syn.: Rhina squatina, A. Dumer., Günth.; Squatina angelus, Blainv., CBp., Canestr.: Squatina vulgaris, Riss., Müll. et Henl., Bocag. et Capel.; Squalus squatina, Lacep., Linn., Bloch; Squatina, Bel., Salvian.

Nom vulgar: Escat ó Angel, Tarragona, Menorca; Angel, Barcelona, Costa de Llevant.

\section{Var. Squatina oculata, Bp.}

Syn.: Rhina aculeata, A. Dumer.; Squatina oculata, Canestr.; Squatina fimbriata, Müll. et Henl.

Nom vulgar: Escat ó Angel, Tarragona; Escat vegigal, Menorca.

Es bastant rara. 
Sub-ordre dels RaITs.

Tribu dels SQUATINORAITS.

Familia dels PRISTIDAE.

Genero, PRISTIS, Latham.

Pristis antiquorum, Latham.

Syn.: Pristis anticuorum, Blainv., Müll. et Heni., A. Dumer., CBp., Günth., Canestr.: Zigaena pristis, Linn.

Nom vulgar: Emperador, Tarragona. Especie no citada en cap Catálech de peixos de Catalunya pero prou coneguda de la nostra gent de mar; es el peix que suara havem dit entaula carnivora y feréstega lluyta amb les especies Oxyrhina Spallanzani, Bp. y Carcharodon Lamia, Bp.

Tribu dels BATIDITs.

Familia dels TORPEDIDAE.

Genero, ToRpedo, C. Dumer.

7 orpedo marmorata, Riss.

Syn.: Raia torpedo, Linn., Blainv.; Torpedo, Couch, Bel.; Torpedo Galvanii, CBp., Canestr., Riss.; Torpedo marmorata, Müll. et Henl., A. Dumer., Günth., Blainv., Cuv.

Nom vulgar: Vaca, Barcelona, Tarragona; Tremuloya, Costa de Llevant; Tremuló, Menorca,

\section{Torpedo oculata, Bel.}

Syn.: Torpedo nurce, CBp., Günth., Canestr.; Torpedo unimaculata, Riss., Blainv.; Torpedo Narke, Riss.; Raia torpedo. Bloch: Torpedo oculata, Müll. et Henl., A. Dumer.; Torpedo, Salvian.

Nom vulgar: Vaca, Barcelona, Tarragona. Moltes d'aquestes va ques no) mostren cap tac 1 . altres solçament ne presenten una ó cinch $y$ d'entre moltissimes d'examinades no més n'havem trobat quatre amb quatre $y$ tres amb tres.

\section{Torpedo nobiliana, Bp.}

Syn.: Torpedo He'betans, Giinth.; Torpedon nohilana, A. Dumer., Canestr.: Torpedo) nigra, Guichenot: Torpedo Walshii, Thompson. 
Nom vulgar: Vaca, Tarragona. Especie nova per Catalunya. En 9 d'Abril de 1910 n'adquirirem un exemplar pescat al bou que midía 0 ‘90 m. de llarch $\times 0$ 0'60 m. d'ampla,'1 20 del mateix mes ne vegerem una altra de tremenda més del doble més grossa y en 18 de Janer de 1911 una altra.

Existeix la preocupació de que subjectant les vaques amb la má esquerra y mossegantse la llengua, un se deslliura dels tremolins ó sacudides eléctriques que descarreguen totes elles.

Familia dels RAIIDAE.

Genero, RaiA, Cuv.

Raia clavata, Rond.

Syn.: Dasybatis clavata, CBp., Canestr.; Raia clavata, Müll. et Henl., A. Dumer., Günth., Lacep., Riss., Blainv.; Raia propia dicta, Bell.

Nom vulgar: Rajada. La var. ocellata: Rajada vera, Tarragona; Clavellada, Costa de Llevant; Clavell, Menorca.

Raia falsavela, A. Dumer.

Syn.: Raia circulariv. Couch, A. Dumer., Günth.; Raia naevus, Muill. et Henl., A. Dumer.; Raia rubus, Lacep.; Raia falsavela, CBp., Canestr.

Nom vulgar: Menja Muxines, Tarragona. Especie no citada en cap Catálech de peixos de Catalunya pero coneguda de nostres pescaires.

Raia naevus, Mü11. et Henl.

Syn.: Raia circularis, Couch, A. Dumer., Günth.; Raia naevus, A. Dumer.; Raia falsavela, CBp., A. Dumer., Canestr.; Raia rubus, Lacep.

Nom vulgar: Menja Muxines, Tarragona. Especie no citada encara lo mateix que 1'anterior.

\section{Raia circularis, Couch.}

Syn.: Raia falsavela, CBp., A. Dumer., Canestr.; Raia naevus, Müll. et Henl., A. Dumer.; Raia circularis, A. Dumer., Günth.; Raia rubus, Lacep.

Nom vulgar: Menja Muxines, Tarragona. Especie com les dugues anteriors no citada en cap Catálech de peixos de Catalunya. 
Raia flossada, Riss.

Syn.: Dasybatis aspera, CBp.; Raia coriácea, Lacep.; Raia chagrinea, Montag., A. Dumer.; Raia fullonica, Linn., Ascanius, Müll. et Henl., Günth., Riss.; Raia aspera nostras, Willugh.

Nom vulgar: Rajada, Tarragona; Clavellada, Costa de Llevant; Clavell-Borrell, Menorca.

\section{Laeviraja Oxyrhynchus, CBp.}

Syn.: Raia Salviani, Müll. et Henl., A. Dumer., Capel.; Raia o.xyrhynchus, Blainv., Günth., Linn.; Laeviraja oxyrhynchus, Canestr.; Raia rostrata, Riss.; Flassade, Rond.

Nom vulgar: Caputxo, Tarragona; Rajada, Menorca.

Laeviraja Macrorhynchus, CBp.

Syn.: Raia rostrata, Blainv.; Laeviraja macrorhynehus, Canestr.; Raia macrorhynchus, Rafin., Gûnth., A. Dumer.; Lentillade, Rond.

Nom vulgar: Moranell, Tarragona; Rajada, Barcelona. Pel mes de Janer es quan se' $n$ agafen més.

Raia batis, Linn.

Syn.: Dasỵbatis Batis, CBp.; Raia batis, Bloch, Blainv., Müll. et Henl., A. Dumer., Günth.

Nom vulgar: Rumiguera ó Romaguera, Tarragona. Especie prou coneguda de tots els nostres pescaires, pero no citada en cap Catálech de peixos de Catalunya. Al bou se' $n$ pesquen que pesen més Ide 700 kilograms.

\section{Raia alba, Lacep.}

Syn.; Laeviraja bramante, Sassi, Canestr.; Raia alba, Blainv.; Raia bicolor, Riss.; Raia laevis major, Duham.

Nom vulgar: Cardaire, Tarragona. Com l'anterior especie prou coneguda y no indicada encara.

$S^{\prime} n$ agafen de tan ó més grosses que les Rumigueres.

Raia marginata, Müll. et Henl.

Syn.: Raia rostellata, Riss.; Raia marginata, A. Dumer., Günth., CBp., Canestr., Lacep., Blainv., Riss.

Nom vulgar: Escrita blanca, Tarragona; Rajada, Barcelona; Claellada, Costa de Llevant; Clavell, Menorca. 
Raiu radula, Del.

Syn.: Batis radula, CBp., Canestr.; Raia virgata, Geof. St. Hil.; Raia radula, Cuv., Blainv., Riss., Müll. et Henl. Guichen., A. Dumer., Günth.

Nom vulgar: Rajada, Barcelona, Tarragona; Clavellada, Costa de Llevant; Rajada peluda, Menorca.

Raia miraletus, Rond.

Syn.: Laeviraja miraletus, Bell.; Raia miraletus, Riss., Blainv., Linn, Müll. et Henl., A. Dumer, 'Günth., CBp., Canestr.

Noın vulgar: Escrita vera, Tarragona; Rajada vestideta, BarceIona; Clavellada, Costa de Llevant; Rajada, Menorca.

Raia Quadrimaculata, Riss.

Syn.: Raia quadrimaculata, CBp., Canestr.

Nom vulgar: Escrita Vera, Tarragona; Rajada de taques, Barce. lona.

Raia punctata, Riss.

Syn.: Dasibatis asterias, CBp., Canestr.; faia Schultii, Müll. et Henl., A. Dumer.; Raia punclata, Günth.; Raia asterias, Del., Blainv.; Asterias sive stellata raia, Bell.

Nom vulgar: Escrita. La var. ocellata Escrita vera, Tarragona; Rajada, Barcelona; Clavellada, Costa de Llevant; Rajada boca de rosa, Menorca.

\section{Raia asterias, Rond.}

Syn.: Raia maculata, Montag., Blainv., CBp., Canestr.; Raia asterias, Müll. et Henl., A. Dumer., Günth.

Nom vulgar: Escrita, Tarragona. Especie nova per Catalunya.

Raia fullonica, Rond.

Syn.: Dasybatis fullonica, CBp., Canestr.; Raia fullonica. Blainv., Riss., A. Dumer., Capel.

Nom vulgar: Rajada, Tarragona, Menorca: Clavellada, Costa de Llevant.

\section{Raia mosaica, Lacep.}

Syn.: Raia undulata, Müll. et Henl., A. Dumer., CBp., Günth., Canestr., Cuv.; Raia mosaica, Blainv. 
Nom vulgar: Escrita vera, Tarragona: Rajada, Barcelona; ClaveIlada, Costa de Llevant.

Tribu dels Cephalop'terienchs.

Familia dels Cephalopteride.

Género, Cephaloptera, C. Dumer.

Cephaloptera Massena, Riss.

Syn.: Cephaloptera Massena, A. Dumer.; Cephalopterus Mas. sena. Riss.

Nom vulgar: Manta Tarragona. Especie nova per Catalunya.

N'havem vist un sol exemplar pescat al bou d'uns 10 kilograms ae pes, peró n'ini ha de molt més grosses que assoleixen més de 3 metres d'envergadura y dificulten moltes vegades el remolch als quillats (barques de bou modernes).

Familia dels MyLIOBATIDE. Género, Myliobatis, C. Dumer. Myliobatis Aquila, C. Dumer.

Syn.: Mỵliobutis nóctula, CBp., Canestr.; Raia uquila, Lacep., Riss., Blainv.. Linn, Bloch; Myliobatis aquila. Cuv., Riss., Müll. et Henl., Guichell., A. Dumer., Capel., Günth.; Aquila de Provença, Duham.: Aquila, Salvian.; Gloriosa, Rond.; Aquila marina, Bell.

Nom vulgar: Milá, Tarragona; Totana ó Totina, Goles de l'Ebre; Milana, Barcelona; Viuda, Menorca.

Familia dels TrYgonide.

Género, Trygon.

Trygon vulgaris, Riss.

Syn.: Trrgon pastinaca, Müll. et Henl., A . Dumer., Günth., CBp., Canestr.: Raia pastinaca, Lacep., Riss., Cuv., Blainv., Linn.; Pas. tinaca hruco, Salvian., Rond.; Pastinaca marina, Bell.

Nom vulgar: Totana ó Totina, Milá, Tarragona; Escursó, BarceIona; Totana ó Totina, Goles de I'Ebre; Escursana, Ferrassa. Menorca.

\section{Trygon violacea, $\mathrm{Bp}$.}

Syn.: Tregon violacea, Müll. et Henl., A. Dumer., Günth., Ca. nestr.

Nom vulgar: Totana ó Totina, Milá, Tarragona; Totana ó Totina, Goles de l'Ebre; Escursó, Barcelona; Escursana, Menorca. 
La classificació d'aquesta familia's fa molt difícil per la precaució que adopten els pescaires de tallar la cua d'aquesta Bastina al tirarla á bordo á causa de lo temible y perillosa que resulta la punxada de la pua dentada que té demunt de la cua y que produheix á vegades accidents mortals.

Algunes n'havem examinat que per les dimensions de la cua'ns semblaren, la Trygon Brucco, Bp., peró no l'incloím en el Catálech pel dupte de poguer esser una mutilació.

Ordre de les CHIMÆREs.

(Holocephals, J. Müller, C. Bonap.)

Familia dels CHIMcERIDE.

Género Chimcera.

Chimora monstruosa, Linn.

Syn.: Artic Chimora, Couch; Chimora mediterranea, Riss., Chimora monstruosa, Lacep., Riss., Cuv,, Bloch, A. Dumer., CBp.; Capel., Günth., Canestr.

Nom vulgar: Caro, Tarragona; Guineu, Torredembarra; UIl vert, Costa de Llevant.

En 23 de Desembre de 1910 ne vegerem á Tarragona una mola de petits ó de cria, tots llarchs d'uns $0.20 \mathrm{~m}$. pescats á l'arrastre (Bou) y en 20 de Janer de 1911 una altra mola de cría.

Secció dels Ganoides.

Ordre dels Esturionits.

Familia dels ACIPENSERIDE.

Género, Acipenser, Arted.

Acipenser Sturio, Linn.

Syn.: Acipenser sturio, Blanchard, Cuv., Riss., Lacep., Bloch, CBp., A. Dumer., Günth., Canestr., Heckel, Siebold; Sturio, Bell.

Nom vulgar: Esturió. Tarragona, Barcelona, Costa de Llevant. Peix de carn exquisita, y que dels ovaris se'n prepara en altres payssos el famós Caviar.

Abunda en 1'Ampolla y'l Fangar (Goles de 1'Ebre); á la Primavera y Estiu se'n pesquen molts de 2 kilograms de pes y á vegades de més grossos. Ríu amunt arriva fins á Xerta. Se 1 pesca també en el Cap de Creus y en altres indrets del litoral. 
Secció de peixos Ichthyostes.

$$
\text { (ossosos) }
$$

(Teleosteenchs, J. Müller.)

Ordre dels LOPHOBRANQUIS, Cuv.

Familia dels Syngnathide.

Sub-familia dels HiPPOCAMPINI, Bp.

Gẻnero, Hippocampus, Cuv.

Hippocampus Guttulatus, Cuv.

Syn.: Syngnathus hippocampus, Bloch; Hippocampus guttulatus, Kaup., CBp., A. Dumer., Günth., Canestr.

Nom vulgar: Cavall mari, Barcelona, Tarragona; Cavallet de mar, Menorca.

Hippocampus brevirostris, Cuv.

Syn.: Hippocampus antiquorum, Günth.: Hippocampus hrevirostris, Kaup., CBp., A. Dumer., Canestr.

Nom vulgar: Cavall marí, Barcelona, Tarragona; Cavallet de mar, Menorca.

Entre la nostra gent de mar els cavalls marins son objecte d:es. pecial preocupació, car se creu que posantsels sota la gorra ó entre'ls plechs del mocador del cap curen la migranya, les neuralgies facials y'l mal de cap (Cefalalgia).

\section{Sub-familia dels SyngnathinI. \\ Género, Syngnathus. \\ Syngnathus rubescens, Riss.}

Syn.: Syngnathus rubescens, Kaup, A. Dumer., Canestr.; Sinhostoma rubescens, CBp.

Nom vulgar: Agulla, Serp, Tarragona. Especie nova per Catalunya.

Syngnathus tenuirostris, Rạthke.

Syn.: S!̣ngnatus tenuirostris, Nordmann, Kaup, A. Dumer., Canestr.

Nom vulgar: Agulla, Serp, Tarragona. Especie nova per Ca. talunya.

\section{Syngnathus Ethon, Riss.}

Syn.: Siphostoma Ethon, CBp.

Nom vulgar: Agulla, Serp, Tarragona. Especie nova per Ca. talunya. 
Syngnatus Abaster, Riss.

Syn.: Syngnathus acus, Riss.; Syngnathus Abaster, Kaup, A. Dumer., Günth., Canestr.; Siphostoma Abaster, CBp.

Nom vulgar: Agulla, Serp, Tarragona. Especie nova per Ca talunya.

\section{Género, Siphonostoma.}

Siphonostoma argentatum, A. Dumer.

Syn.: Siphonostomus tuphle, Canestr.; Siphonostomus argen. tatus, Kaup; Syngnatus argentatus, Pallas, Rathke, Nordmann.

Nom vulgar: Agulla, Serp, Tarragona. Especie nova per Catalunya. Bastant rara.

\section{Siphonostoma Rondeletii, Kaup.}

Syn.: Syngnathus viridis, Riss.; Syngnathus Ro deletii, Bel.: Siphonostoma Rondeletii, Canestr., A. Dumer.

Nom vulgar: Agulla, Serp, Tarragona; Musich, Barcelona; Serp d'en terra ó de costa, Costa de Llevant; Peix bade Menorca.

\section{Var. Siphonostoma pyrois, A. Duner.}

Syn.: Syngnathus pyrois, Riss.; Siphonostomus pyrois, Kaup.

Nom vulgar: Agulla, Serp, Tarragona; Musich, Barcelona; Serp d'en terra ó de costa, Costa de Llevant.

Sub-familia dels NEROPHINI.

Género, Nerophis, RAFin.

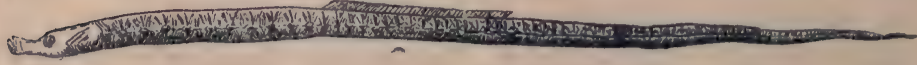

Nerophis lumbriciformis, Bp.

Syn.: Acus lumbriciformis aut serpentinus, Willugh.; Nero phis lumbriciformis, Kaup, A. Dumer., Günth.

Nom vulgar: Serpeta, Tarragcna. Especie nova en el Mediterrá de la qual ne servem un exemplar, l'unich que havem vist, pescat á l'artet el primer de Abril de 1911.

\section{Nerophis Ophidion, Bp.}

Syn.: Syngnathus Ophidion, Linn., Riss.; Scyphius littoralis, Riss.; Nerophis óphidion, Kaup, A. Dumer., Günth.. Canestr. 
Nom vulgar: Serpeta, Tarragona. Especie nova per Catalunya.

El Siphostoma acus, L. que cita en el seu Catảlech en Sanchez Comendador, si es el S!̣ngnathus acus, L., devém confesar que no I'havém pogut trobar, será tal vegada alguna de les especies suara citades.

Ordre dels Plectognaths.

Sub-ordre dels Gymnodonts, Cuv.

Familia dels OrTHAGORISCIDAE.

Género OrthagORISCUS.

Orthagoriscus Mola, Schneid.

Syn.: Diodon Mola, Pallas; Orthagoriscus spinosus, B1. Sch neid., Cuv., Gatchet; Mola aculeata, Kölreuter; Mola aspera CBp.; Cephalus orlhagoriscus, Riss.: Cephalus mola, Riss.; Or. Ihugoriscus mola, Lacep., Günth., Canestr.: Tetrodon mola, Linn., Bloch; Luna ó mola, Salvian., Duham. Rond.

Nom vulgar: Bot, Barcelona, Tarragona. Els magres y'ls budells d'aquest peix son molt estimats dels gurmets.

En 18 de Juny de 1910 l'art de la Platja del Miracle (Tarragona) en un sol vol n'agafá tretze y altres tants al día seguent y n'havém vist de tan grossos y vells que les barques no'ls poden issar sobre cuberta y tenen de remolcarlos y treurels arrastrats per cavalleries; el cos d'alguns d'ells está recubert de vistoses algues y moluschs com el plá o'ls fons bruts d'un vaixell.

\section{Sub-ordre dels ScLERODERMS. \\ Familia dels BALISTIDE. \\ Género, BaListes, Linn. \\ Balistes capriscus, Linn.}

Syn.: Balistes lunulatus, Riss.; Ealistes Buniva, Lacep., Riss.; Balistes caprisc'us, Gml.. Agrass., Günth., CBp., Canestr., Hollard, Lacep.. Riss.; Capriscus, Salvian., Wiilugh.; Caper, Salvian.; Porc', Rond.

Nom vulgar: Ballesta, Tarragona. Especie rara. Servem la pell d'un d'aquests peixos agafat á l'almadrava que's calava anys enrera á la punta de Pó-roig del roquer de Salou que ben bé podría ferse servir de capell, y pescat á l'art prop de la Torra de la Mora á 6 de Maig de $1911 \mathrm{n}$ adquirirem un altre exemplar que servem preparat. 
Ordre dels ChORignaths.

Sub ordre dels ACANTHOPTERYGIS.

Tribu dels ACANTHOPTERYGIS JUGULARS.

Familia dels TrachinIDE.

Género, URANoscopus, Linn.

Uranoscopus scaber, Linn.

Syn.: Uranoscopus scaber, Cuv. et Valenc., Cuv., Guichen., Lacep., Riss., Bloch, Günth., CBp., Canestr.; Uranoscopus, Salvian.; Callionimus sive uranoscopus, Bell.

Nom vulgar: Rata, Tarragona, Barcelona; Salteperdisa, Rata, Menorca.

Género, Trachinus, Arted.

Trachinus vipera, Cuv.

Syn.: Viper Weever, Couch; Trachınus vipera, Günth., CBp., Canestr., Cuv. et Valenc.; Araneola. Duham.

Nom vulgar: Aranyó. Especie no citada en cap Catálech de peixos de Catalunya pero coneguda de nostres pescaires.

Trachinus draco, Linn.

Syn.: Trachinus vividus, Lacep.; Trachinus draco, Cuv. et Valenc., Günth., CBp., Canestr., Riss.; Vive, Duham., Rond.; Draco marinus, Bell.

Nom vulgar: Aranya, Barcelona, Tarragona; Aranya blanca, Dragó, Menorca.

\section{Trachinus radiatus, Cuv.}

Syn.: Trachinus draco, Brunnich; Trachinus radiatus, CBp., Günth., Canestr.; Trachinus lineatus, Bel.

Nom vulgar: Aranya, Tarragona; Aranya capsuda, Barcelona; Aranya de cap negre, Menorca.

\section{Trachinus araneus, Cuv.}

Syn.: Draco major, Salvian, Willugh.; Trachinus araneus, CBp.; Günth., Canestr., Agass.

Nom vulgar: Aranya, Barcelona, Tarragona; Aranya fragata, Menorca.

Les punxades de les Aranyes ocasionen greus accidents: inflamacions, eixidures (flemons) y á vegades erissipeles gangrenoses 
(flemons difusos). Segons els pescaires son més verinoses en lluna nova y amb les correnties de llevant.

Familia dels BLENNIIDF.

Género, Blennius, Arted.

Blennius Pavo, Riss.

Syn.: Ichthyocoris Pavo, CBp.; Blennius galerita, Lacep., Riss.; Blennius pavo, Günth., Canestr., Cuv. et Valenc.; Galerita, Rond.

Nom vulgar: Dormilega, Tarragona; Capsigrany, Barcelona; Futarra, Bavosa, Costa de Llevant; Gallerbu, Menorca.

\section{Blennius palmicornis, C. V.}

Syn.: Blennius Pholis, Riss.; Blennius sanguinolentus, Pallas, Günth.; Blennius palmicornis, CBp., Canestr.

Nom vulgar: Dormilega, Tarragona; Capsigrany, Llepissós, Barcelona; Futarra, Bavosa, Costa de Llevant; Rabosa, Menorca.

\section{Blennius Cagnota, Valenc.}

Syn.: Blennius varus, Gûnth.; Blennius vulgaris, Günth., Canestr.; Ichthyocoris anticolus, CBp.; Ichthyocoris Cagnota, CBp.; Ichtivocoris varus, CBP.; Blennius alpestris, Blanch.; Blennius Cagnota, Heckel et Kner, Cuv. et Valenc., Blanch.; Salurias varus, Riss.; Blennius sujefianus, Riss.

Nom vulgar: Barb cani, Comarques gironines. Especie nova per Catalunya, més ben dit, no indicada en cap Catálech de peixos de Catalunya.

En el Laboratori ictiogènich del Park de la Ciutadela de Barcelona 'ns la mostrá d'aquesta procedencia 'I seu director en Francesch d'A. Darder.

Blentius gattorugine, Lacep.

Syn.: Gattorugine, Couch, Willugh.; Blennius gattorugine, Riss., Cnv. et Valenc., Guichen.; Brunnichii, CBp., Günth., Canestr.

Nom vulgar: Dormilega roija ó de fang, Tarragona; Capsigrany, Barcelona: Futarra, Babosa, Costa de Llevant; Rabosa, Menorca. 
Blennius tentacularis, Brunn.

Syn.: Blennius Brea, Riss.; Blennius cornutus, Riss.; Blen. nius punctulatus, Riss.; Blennius tentaculatus, Lacep., Riss.; Blennius tenlacularis, Cuv. et Valenc., Guichen., CBp.,Günth., Canestr.

Nom vulgar: Dormilega, Tarragona; Capsigrany, Barcelona; Futarra, Babosa, Costa de Llevant; Rabosa, Menorca.

Blennius ocellaris, Linn.

Syn.: Blennius vel capola, Bell.; Blennus, Salvian.; Blennus Salviani, Willugh.; Blennius ocellaris, Bloch, CBp., Günth., Canestr., Lacep., Riss., Cuv. et Valenc.

Nom vulgar: Dormilega, Tarragona; Capsigrany, Ase mossegai re, Barcelona; Futarra, Bavosa, Costa de Llevant; Rabosa, Menorca.

Blennius Erythrocephalus, Riss.

Syn.: Ichthyocoris rubriceps, CBp.; Blennius rubriceps, Cuv. et Valenc.; Blennius ervthrocephalus, Günth., Canestr., Riss.

Nom vulgar: Dormilega de cap roig, Tarragona; Capsigrany. Barcelona; Futarra, Babosa, Costa de Llevant; Rabosa, Menorca.

\section{Blennius Sphinx, Valenc.}

Syn.: Blennius sphinx, CBp., Günth., Canestr., Cuv. et Valenc., Guichen.

Nom vulgar: Dormilega petita ó de roca, Tarragona. Especie nova per Catalunya.

\section{Blennius incequalis, Valenc.}

Syn.: Blennius inoequalis, Cuv. et Valenc., CBp.

Nom vulgar: Dormilega, Tarragona. Especie nova per Catalunya.

Servém tres exemplars recullits en 1. cr d'Agost de 1910 d'una especie nova ó varietat de l'especie anterior, però sense'ls tentáculs de les celles y dels forats nassals. Tenen $0,04 \mathrm{~m}$. de llarch y llur pell no es tan llepissosa; liur color á poca diferencia es el mateix y la dorsal es desigual, amb el mateix número de ratjs forts y molls, així com l'anal y les ventrals, emprò la caudal te més de deu ratjs. Les peçes dentaries. dalt y baix, son iguales. Pudrien ser també petits exemplars de la Blennius basíliscus, Valenc. 
Familia dels Callionymide, Bp.

Género, Callionymus, Linn.

Callionymus lyra, Linn.

(Mascle gran.)

Syn.: Callionymus lyra, Lacep., Cuv. et Valenc, Guichen, CBp., Günth., Canestr.

\section{(Femella.)}

Syn : Cullionymus dracunculue, Lacep., Bloch, Cuv. et Valenc., Guichen.

Nom vulgar: Guineu vermella, Tarragona; Dragó, Menorca. Especie rara.

La Callionymus elegans,Lesueur; es la petita ó de cria (Callio nimus Sueurii, Cuv. et Valenc.)

Callionymus maculatus, Rafin.

Syn.: Callionymus cithara, Cuv. et Valenc.; Callionymus lyra, Riss.; Callionymus maculatus, CBp., Günth., Canestr.; C'allionymus Dracunculux, Brunn.

Nom vulgar: Guineu, Tarragona; Pau, Barcelona; Ferrepedres, Menorca.

\section{Callionymus Dracunculus, CBp.}

Syn.: Callionymu. lncerta, Cuv, et Valenc., Guichen.; Callio nymus Dracunculus, Canestr.; Callionymus admirabilis, Riss.; Callionymu× pusillu., Del., Riss.; Callionymus testivus, Pallas, Nordmann; Dracunculus, Gesner, Willugh., Rond.

Nom vulgar: Guineu, Tarragona. Especie nova per Catalunya.

Callionymus Belenus, CBp.

Syn.: Callion!mus Morissonii, Riss., Canestr.; Callionymus Risso, Lesueur, Cuv. et Valenc.; Callionymus Belenus, Günth. Canestr., Cuv. et Valenc., Riss.; Callionvmus sagitta, Riss.; Belen uus, Rond.; Blennus vel, Belennus, Gesner.

Nom vulgar: Guineu, Tarragona; Pau, Barcelona; Cabot en banyes, Menorca.

Se 'n troben bastantes entre 'I Xanguet ò Carmelo ros (Apria pellucidu, Moreau.) 
Familia dels LoPHIDÆ.

Género LopHIUs, Arted.

Lophius piscatorius, Linn.

Syn.: Batrachus píscatorius, Riss.; Rana marina, Bell.; Galanga, Rond.; Rana piscatrix, Willugh.; Lophius piscatorius, Riss., Cuv. et Valenc., Lacep., Bloch, CBp., Canestr., Günth.

Nom vulgar: Rap, Barcelona, Tarragona; Rap, Buldroi, Menorca.

\section{Ĺophius Budegassa, Spinola.}

Syn.: Lophius parvipinnis, Cuv.; Lophius Budegassa, Riss, CBp., Günth., Canestr.

Nom vulgar: Rap futaire, Tarragona; Rap, Barcelona.

En el pahidor d'alguns raps els hi havem trobat sencer el molusch, Scaphander lignarius, Linn.

Tribu dels AcANTHOPTERygis

thoracichs.

Familia dels GoBIIDÆ.

Género, GobIUs, Arted.

Gobius Jozo, Linn.

Syn.: Gobius nebulosus, Riss.; Gobius tertius, Willugh.; Gobius Jozo, Cuv. et Valenc., Lacep., Riss., CBp., Canestr., Günth.

Nom vulgar: Ruch d'alga, Tarragona; Burru, Barcelona; Gobit d'alga, Costa de Llevant; Cabot d'alga, Menorca.

Var. Gobius longiradiatus, $\mathrm{CBp}$.

Syn.: Gobius longiradiatus, Riss., Cuv. et Valenc.

Nom vulgar: Ruch, Tarragona; Burru, Barcelona; Gobit, Costa de Llevant; Cabot mucós, Menorca.

Gobius lota, Valenc.

Syn.: Gobius ophiocephalus, Pallas, Günth.; Gobius lota, CBp., Canestr., Cuv. et Valenc.

Nom vulgar: Ruch, Tarragona. Especie nova per Catalunya.

Gobius Guttatus, Cuv. et Valenc.

Syn.: Gobius guttatus, CBp., Canestr.

Nom vulgar: Ruch de roca, Tarragona; Gobit de roca, Costa de Llevant; Cabot de roca, Menorca. 
Gobius cruentatus, Gm1.

Syn.: Gobius cruentatus, Lacep., Riss., Cuv. et Valenc., Guichen., Linn., CBp., Günth., Canestr.

Nom vulgar: Ruch, Tarragona; Burru, Barcelona; Gobit, Costa de Llevant; Cabot inglés, Menorca.

Gobius quadrimaculatus, Valenc.

Syn.: Gobius aphia, Riss.; Gobius quadrimaculatus, Cuv. et Valenc., Guichen., CBp., Canestr.

Nom vulgar: Ruch, Tarragona. Especie nova per Catalunya.

Gobius minutus, Cuv, et Valenc.

Syn.: Gobius elongatus, Canestr.; Gobius minutus, CBp., Günth., Guichen.

Nom vulgar: Ruch, Tarragona; Gobit, Costa de Llevant; Cabot, Menorca.

Gobius auratus, Riss.

Syn.: Gobius auratus, Cuv, et Valenc., CBp., Günth., Canestr.

Nom vulgar: Ruch, Tarragona. Especie nova per Catalunya.

Se'n troben molts entre'l carmelo (Meletta Phalerica, M.; Alattsa Pilchardus, Cuv, et Valenc.)

Gobius geniporus, Valenc.

Syn.: Gobius geniporus, CBp., Günth., Canestr., Cuv. et Valenc.

Nom vuigar: Ruch, Tarragona; Cabot, Menorca.

'Gobius Paganellus, Linn.

Syn.: Gobius punctipinnis, Canestr.; Paganellus Venetorum, Willugh.; Gobius paganellus, Cuv. et Valenc., Guichen., Brunn., CBp.

Nom vulgar: Ruch, Tarragona; Burru, Barcelona; Gobit, Costa de Llevant; Cabot, Menorca.

Gobius niger, Linn.

Syn.: (iohius niger, Cuv. et Valenc., Bloch, CBp., Günth.

Nom vulgar: Ruch de roca, Tarragona; Gobit de roca, Costa de Llevant, Cabot de roca, Menorca. 
Gobius Fallax, Sarato.

Nom vulgar: Ruch, Tarragona; Cabot Berrubes, Menorca.

Género, ApHia, Riss. Aphia Pellucida, Moreau.

Syn.. Aiherina minuta, Riss.: Latrun 'ulus pellucidus, Günth.: Gobius albus, Canestr.; Brachvochirus Aphia, CBp.; Brachyochirus Pellucidus, Nardo; Gobius Pellucidus, Kessler, Nardo; Aphiu meridionalis, Riss

Nom vulgar: Xanguet, Barcelona; Carmelo ros, Tarragona; Llenqueta, Torredembarra; Roseti. Menorca.

Especie coneguda de tothom y no indicada en cap Catalech de peixos de Catalunya.

Familia dels MULLID E.

Género, Mullus, Linn.

Mullus surmuletus, Linn.

Syn.: Surmuletus, Couch; Mullus surmuletus, Cuv. et Valenc. Lacep., Riss., Bloch, CBp., Günth., Canestr.

Nom vulgar: Roger, Rogeret, Costa de Llevant; Moll-fangué, Tarragona; Moll ve, Menorca.

Mullus fuscatus, Rafin.

Syn.: Mullus fuscus, Riss.

Nom vulgar: Moll, Tarragona, Menorca; Roger, Rogeret, Costa de Llevant.

Mullus Barbatus, Willgh.

Syn.: Mullus ruber, Riss.; Mullus, Salvian.; Mullus barbatus, Cuv. et Valenc., Lacep., Linn., Bloch, CBp., Günth., Canestr.

Nom vulgar: Roger, Rogeret, Costa de Llevant; Moll roquer, Tarragona; Moll cranqué, Menorca.

En 13 d'Agost de 1910 vejerem un petit moll roquer deformat: presentava un gep ben marcat y n'adquirirem un altre de semblant en 22 d'Octubre del mateix any; abdos patien una malaltia ó incorvació de la columna vertebral, mes ben dit, una de les dugues formes de l'afecció anomenada ciphosis ó-siga la ciphosis arciforme (juvenil) raquítica, no la spondilica angular ó ciphosis de Pott. 
Familia dels TRIGLIDE.

Género, Dactylopterus, Lacep.

Dactylopteras volitans, CBp.

Syn.: Dactylopterus pirapcda, Lacep., Riss.; Dactylopterus volitans, Günth., Canestr., Cuv. et Valenc., Guichen.; Trigla volitans, Linn., Bloch; Milvas, Bell.

Nom vulgar: Xuriguer, Tarragona; Xuriguer, Marmota, BarceIona; Juriola voladora, Costa de Llevant; Xorich volador, Menorca.

Género, Peristedion, Lacep.

Peristedion Cataphractum, CBp.

Syn.: Peristethus cataphractum, Günth.; Peristedıon cataphractum, Brit., Canestr.; Peristedion cataphractus, Riss.; Peristedion malarmat, Lacep., Rișs.; Peristedion chabrontera, Lacep., Riss.; Trigla chabrontera, Bonnat.; Trigla cataphracta, Linn., Bloch, Brunn.; Malarmat, Bell., Rond., Duham., Bonnat., Cuv. et Valenc.

Nom vulgar: Malarmat, Barcelona, Tarragona; Burru, Costa de Llevant; Armat, Menorca. Peix de fonera.

Género, Trigla, Arted.

Trigla pini, Bloch.

Syn.: Trigla pini, Lacep., Riss., Günth.

Nom vulgar: Peona, Tarragona; Lluerna pogona, Barcelona; Gallineta, Menorca.

Trigla lineata, Wall.

Syn.: Trigla adriática, Gml., Riss.; Trigla lineata, Bloch, CBp., Günth., Canestr.; Imbriago, Rond.; Trigla (Lastoviza), Brunn., Lacep.

Nom vulgar: Borratxo, Tarragona; Rafalet, Menorca.

Trigla cuculus, Rond.

Syn.: Trigla lucerna, Cuv. et Valenc., Guichen., Brunn., Riss.; Trigla cuculus, Riss.; Trigla obscura, Linn., CBp., Günth., Canestr.

Nom vulgar: Lluerna, Baı celona, Tarragona; Gallineta, Menorca.

Trigla gurnardus, Linn.

Syn.: Trigla gurnardus, Cuv. et Valenc., Bloch, CBp., Günth., Canestr.; Trigla gurnutu, Lacep,, Riss. 
Nom vulgar: Refet, Refetó, Tarragona; Lluerna, Biret, Bar celona.

Al Refetó petit o Refetonet se li dona'l nom de: Pelut, Capsech, Clau, Tarragona: Clavilló, Costa de Llevant, Goles de l'Ebre.

\section{Var. Trigla milvus, CBp.}

Syn.: Trigla cuculus, Cuv. et Valenc., Bloch, Günth.; Trigla hirundo, Brunn.; Trigla milvus, Canestr., Riss.; Milvus, Rond.

Nom vulgar: Lluerna, Tarragona. Var. nova per Catalunya.

$$
\text { Trigla Lyra, Lacep. }
$$

Syn.: Trigla Lyra, Cuv. et Valenc., Riss., Guichen., Bonnat., Linn., Bloch, CBp., Canestr., Günth.; Lyra, Rond.

Nom vulgar: Gatneu, Tarragona; Garneu, Barcelona, Costa de Llevant; Ase, Menorca.

Al petit o de cria se li dona'l nom de Gatneu futaire, com si fos una especie distinta.

Peix de fonera, molt abundant y que com en el Rap li havém trobat sencer en el seu pahidor el molusch Scaphander lignarius, Linn.

\section{Trigla corax, $\mathrm{CBp}$.}

Syn.: Trigla corax, Canestr.; Trigla microlepidota, Riss.; Trigla corvus, Riss.; Trigla hirundo, Cuv. et Valenc., Lacep., Guichen., Bloch, Günth.; Trigla cuculus, Brunn.; Corax, Rond.

Nom vulgar: Juriola, Tarragona, Costa de Llevant, Menorca; Lluerna, Barcelona.

La petita o de cria es la:

Trigla pociloptera, Valenc.

Syn.: Trigla pœciloptera, Guichen., Günth. norca.

Nom vulgar: Lluerneta, Juriola de cria, Tarragona; Oriola, Me-

Avans de perdre-s la navegació costel'a a la vela y d'aumentar el nombre de les barques de pesca, abundava moltissim més que'n nos. tres dies.

$$
\text { Trigla cavillone, Lacep. }
$$

Syn.: Lepidotrigla aspera, Günth., Sauvage; Trigla aspera, 
CBp., Canestr., Viviani, Cuv. et Valenc., Guichen.; Trigla cavíllone, Riss.: Mullus asper, Rond.

Nom vulgar: Pelut d'escata, Tarragona; Escatós, Goles de l'Ebre; Clavilló, Costa de Llevant.

Peix de fonera abundant com el Gatneu y encara que de baix preu, no menys estimat pera menjar a la brasa.

Sub-familia dels CotTint.

Género, CotTus, Arted.

Cottus gobio, Linn.

Syn.: Gobius fluvialis alter, Bell.; Chabot, Rond., Duham., VaIlot; Cottus gobio, Bloch, Jurine, CBp., Günth., Canestr., Heckel et Kner.. Siebold, Gehin, Lacep., Riss., Cuv. et Valenc., Blanch., Soland.

Nom vulgar: Cullereta, Canal d'Urgell. Especie nova per Catalunya.

\section{Sub-familia dels SCORPENINI. \\ Gẻnero ScORP ENA Linn. \\ Scorpæna, scropha Linn.}

Syn.: Scorpitus, Salvian.: Scorpio marınus, Bell.; Scorpcena scrofa, Bloch, Brunn., Costa, CBp., Günth., Canestr., Lowe, Sauvage, Lacep., Riss., Cuv. et Valenc., Guichen.

Nom vulgar: Polla, Escórpora dels bruts, Escórpora de fang, Tarragona; Polla, Barcelona; Cap roig, Menorca.

$$
\text { Var. Scorpæna lutea, Riss. }
$$

Nom vulgar: Escorpora groga, Tarragona. Nova per Catalunya. Rara:

\section{Scorpana porcus, Linn.}

Syn.: Scorpana porcus, Cuv. et Valenc., Guichen., Lacep., Riss. . Bloch, Brunn., Costa, CBp., Günth., Canestr., Sauvage; Scorpana, Bell.

Nom vulgar: Escórpora de roquer, Tarragona, Barcelona; Ras cla, Menorca.

Scorpona ustulata; Lowe.

Syn.: Scorpona porcus, Costa; Scorpana usıulata, Günth., Giglioli, Belloti. 
Nom vulgar: Escórpora de roquer, Tarragona; Cap-tinyós, Uot, Menorca.

Les punxades de les Escórpores son dolentes, però no tan com les de les Aranyes (Trachinus). Amb la fosca y les correntíes de llevant, son aquestes més verinoses.

En Desembre de 1909 adquirirem un petit exemplar de Scorpana que segurament es una varietat de la S. ustulata, Lowe, car en lloch de presentar 18 o 19 ratjs en les pectorals, no més ne té 13 o 14 y la coloració es d'un hermós color de cendre amb grans taques blanques y negres, sobre tot una de blanca a la basa o arrancada de la cua y les puntes de les aletes son vermelloses, No la considerém especie nova per haver arribat a les nostres mans molts exemplars de Scorpona ustulata, Lowe, amb 16 ratjs en lès pectorals.

Género, Sebastes, Cuv.

Sebastes Dactytopterus, Günth.

Syn.: Sebastes imperialis, CBp., Canestr.; Scorpana dactylop. tera, Laroche, Riss.; Serranus imperialis, Cuv. et Valenc., Guichen. y dels Mallorquins.

Nom vulgar: Rufina, Costa de Llevant; Panegal, Tarragona; Panegall, Barcelona; Serrá imperial, Menorca.

Peix de fonera, molt abundant.

Familia dels BERYGIDE.

Género, Hoplostethus, Cuv.

Hoplostetus mediterraneus, Cuv. et Valenc.

Syn.: Trachichthys pretiosus, Lowe; Trachichthys australis, Costa; Trachichthys mediterraneus, CBp.; Hoplostethus mediterraneus, Guichen., Günth., Canestr.

Nom vulgar: no'n té. Especie molt rara y nova per Catalunya. N'havèm vist dos exemplars procedents de Badalona, en casa de nostre amich el naturalista preparador en Lluis Soler y Pujol, de Barcelona.

Familia del PERCID E.

Género, LABRAx, Cuv.

Labrax Lupus, Cuv.

Syn.: Centropomus, Lacep.; Perca Labrax, Riss., Linn., Brunn.; Labra.x lupus, Cuv. et Valenc., Guichen., CBp., Günth., Canestr., 
Brit. Capel.: Bur, Duham.; Sciaena diacantha, Bloch; Lupus, Bell., Rond., Bonnat.

Nom vulgar: Llubarro, Llubina. Barcelona, Tarragona, Menorca; Llop, Goles y riberes de l'Ebre, Menorca,

En 29 d'Abril de 1910, dintre'l port de Tarragona, se'n pescá amb am un d'aquests peixos de mes de un metre de llarch.

\section{Labrax punctatus, B, C}

Syn.: Labrax punctatus, L. Vaillant.

Nom vulgar: Baila, Tarragona; Llop, Menorca.

$$
\begin{gathered}
\text { Sub - familia dels SERRANini. } \\
\text { Género, Polyprion, Cuv. } \\
\text { Polyprion cernium, Valenc. }
\end{gathered}
$$

Syn.: Sciaena aquila, Rosenthal; Polvprion Massilien se, Costa; Polyprion cernier, Lowe; Polyprion cernium, CBp., Günth., Ca nestr., Cuv. et Valenc.; Holocentrus Gulo, Riss.; Scorpaena mas. siliensis, Risı..: Meru del Cap Breton, Duham.

Nom vulgar: Rascás, Tarragona; Dot, Barcelona; Pampol, Rascás. Menorca.

$\mathrm{Se}$ 'l veu quasi sempre en alta mar, junt a les desferres d'embar. cacions y fustes surants.

\section{Género, Serranus \\ Serranus Scriba, CBp.}

Syn.: Lutjanus scriptura, Lacep., Riss.: Holocentrus marinus, Lacep., Del., Riss.; Holocentrus argus, Riss.; Serranus Scriba, Cuv. et Valenc., Guichen., Günth., Canestr.; Perca marina, Salvian., Willugh., Brunn.

Nom vulgar: Vaca Serrá, Barcelona, Tarragona; Vaca serrana, Goles de I'Ebre; Vaca, Menorca,

Serranus cabrilla, CBp.

Syn.: Holocentrus virescens, Bloch; Holocentrus chanus, Lacep.: Hólocentrus flavus, Riss.; Bodianus hiatula, Lacep.; Serranus cabrilla, Riss., Cuv. et Valenc., Günth., Canestr.; Perca ma rina, Brunn.; Perca cabrilla, Linn.; Hiatula, Salvian.; Comber, Couch.

Nom vulgar: Serrá, Barcelona, Tarragona, Menorca. 
Serranus heputus; CBp.

Syn.: Centropristis hepatus, Günth.; Serranus hepatus, Canestr., Riss., Cuv. et Valenc., Guichen.; Holocentrus hepatus, Riss.; Holocentrus siagonotus, Del.; Lutjanus adriaticus, Lacep.; Holocentrus striatus, Bloch; Labrus, Brunn.; Labrus heputus, Linn., Lacep.; Sachettus Venetorum, Willugh.

Nom vulgar: Inflaconys, Tarragona; Serrá, Barcelona; Vaca serrana, Menorca.

Género, Cerna, Bp. (EPINEPhelus, Bloch.)

Cerna gigas, CBp.

Syn.; Epinephelus gigas, Vaill., Br.; Serranus gigas, Günth., Canestr., L. Vaillant, Cuv. et Valenc., Guichen., Riss., Geof. St. Hil.; Holocentrus, Blainv.; Holocentrus meru, Lacep., Riss.; Perca gigas, Bonnat., Brunn., Linn.; Serratus marginatus fimbria tus, Lowe; Cernna gigas, Costa; Perca cernua, Aradas.

Nom vulgar: Meru, Tarragona; Meru, Neru, Barcelona, Custa de Llevant; Anfós, Menorca.

Peix de carn delicada, molt recercat pêls gurmets.

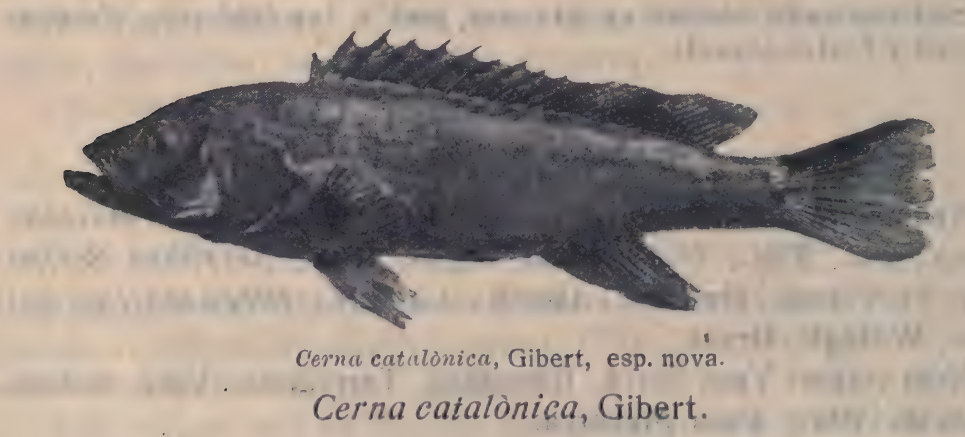

Nom vulgar: Abadeco ratllat, Tarragona.

La forma de la cúa es $\in \mathrm{I}$ caràcter de classificació més essencial del genero Meru o Epinephelus, Bloch (Cerna, Bp.). Cal, per tant, modificar el quadro y afegir hi, a mes dels de cúa rodona y de cúa partida, els de cúa recta $\delta$ quadrada.

Aquesta especie no es cap Serrá; pera serho li manca presentar la vora inferior del preopercul dentellada en la seva meytat posterior y les escates de la línea mitja dels costats, ciliades y no llises. 
Sobra, per tant, dir que siga una varietat del Vaca-Serrá (Serranus scriba, Cuv. et Valenc.) que té la cúa recta o quadrada.

Per ésser el Plectropoma fasciatum, Costa (Cerna alexandrina, Doderl.; Serranus alexandrinus, C. V.; Serranus acutirostris, Perugia), li manca á nostra Cerna, en primer lloch: portar á l'angle del preopercul les dents més ó menys grosses que dirigides oblicua ment endevant constituheixen el carácter del género Plectropoma, Cuv., y en segón lloch, tenir la cúa lleugerament partida.

La Cerna catalónica (fusciata, recticerca) es un peix de la familia dels Serranienchs; té'l preopercul dentellat y les barres (mandíbula) escatoses; es per tant més una Cerna ó Epinephelus que un Serrá. Té la vora inferior del preopercul, recta y sense dentellons. Veusen aqui els seus carácters:

Dorsal, 11/16 ; Anal, 3/8 ó 7; Pectorals, 15; Ventrals, 1/5. Dels cinch exemplars recullits en tres anys, no més ne serven tres, dos de preparats y la fotografía d'un altre, tots no passen gayre de $0^{\text {‘ } 22 ~ o ́ ~}$ $0.23 \mathrm{~m}$. de llarch; l'alçaria del cos es de 0.04 m.; la llargada del cap de $0.06 \mathrm{~m}$. ó siga ${ }^{1}{ }_{3}$ mes llarch que la alçaria y compresa encara no, quatre vegades en la llargaria total; té ademés de les dents en carda, dos ullals dalt y baix. El ratj fort o punxós de les ventrals es més llarch que la meytat del ratj següent; la vora inférior del subopercul no es dentada; les dents de l'angle inferior del preopercul son desiguals; la caudal es sempre recta ó quadrada. Aquests son els carácters principals d'aquesta nova especie per Europa, a la que'ns havèm permès posar el noin de Cerna catalónica. El color del cos es roijós ab 5 ratlles blavench negroses longitudinals en els costats, y 2 del mateix color obliquies en el preopèrcul.

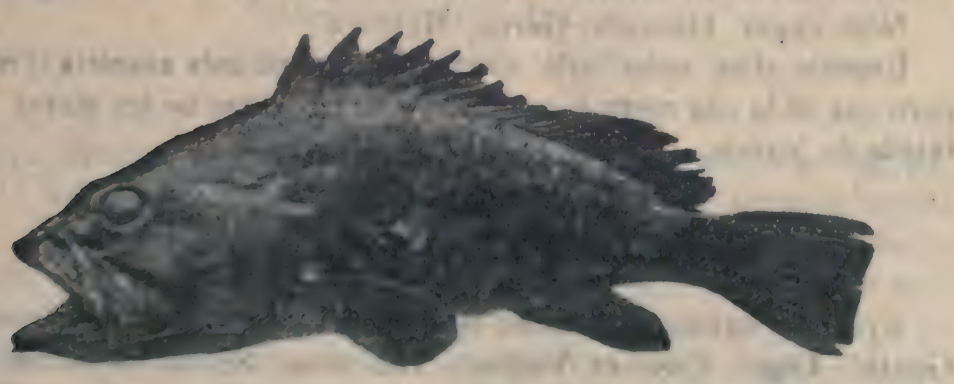

Cierna canina, Inder1. 


\section{Cerna canina, Doderl.}

Syn.: Serranus caninus, Val.; Cerna gigas, Gthr., Stdchnr.; Serranus cernioides, B. Capel.

Nom vulgar: Meru, Tarragona; Gernera, Gerna, Menorca. Especie nova per Catalunya.

El día 10 de Maig de 1911 ens arribà a les mans un bell exemplar de Meru, qual carâcter essenciai de diferenciació respecte'ls altres, consistía en tenir la cúa recta o quadrada. El peix adquireix grans proporcions, car el nostre exemplar midía $0,50 \mathrm{~m}$. de 1 larch $\times 0$ '23 m. d'alt, y pesava 1,500 grams.

Aquesta nova especie no deu confondre-s amb la nostra Cerna catalónica, puix que es ratllada y sempre de més petites dimensions, ni amb la Cerna gigas, CBp., ni amb l'Epinephelus acutirostris, C. et V., car la C. gigas té la caudal rodona y'l darrer la té partida. Els caràcters son quasi'Is mateixos de l'especie Gigas, però com ja tením índicat, té la caudal recta ó quadrada; els dentellons angulars del preopèrcul son quasi iguals y la vora inferior del subopercul es finament dentellonada. El color es poch més ó menys el de la Cerna Gigas Bp.; el no ésser com havèm dit ratllat, el diferencía de la $C$. catalónica, $G$., y el comptar no més amb 8 ratjs molls en en l'anal, fá que no se la pugui confondre amb l'E. acutirostris, C. et $\mathrm{V}$. que constantment ne té 11 y ensemps la cúa partida,

Br: 7 -D. $11 / 15 ;$ A. $3 / 9$; C. $15 \cdot$ P. 17 ; V. $1 / 5$.

Unicament pot confondre $\mathrm{s}$ amb la:

\section{Cerna sicana, Doderl.}

Syn.: Centropristimero et Cerna nigrita, Jordan et Eigemann.

Nom vugar: Gernera, Gerna, Menorca.

Especie d'un color gris vermellós que no més assoleix 0‘86 m., però que té la cúa recta ó quadrada y l'extremitat de les aletes ribetejada de blanch.

$$
\text { D. } 10 / 15 ; \text { A. } 3 / 9 \text {. }
$$

Epinephelus acutirostris, C. et V.

Syn.: Serranus nudulosus. Steindachn.; Serranus acutirostrís, Günth., Gigl., Cuv. et Valenc., Guichen.; Serranus nebolosus, Cocco; Serranus tinca, Cantraine; Serranus fuscus, Lowe, Steiri- 
dachn.; Cerna nehulosa. CBp., Verany; Serranns macrogenis, Sassi, Gigl.

Nom vulgar: Abadeco, Tarragona. Especie rara.

Abunda mes vers les aygues de Ponent y de l'Estret.

No ha arribat a les nostres mans y per tant no havèm pogut examinar cap exemplar de l'especie de cúa lleugerament partida color pardo amb bandes en el cos y cap, y taques vert-groguenques en els costats y d'un tò més clar en el ventre, que cita en Jaume Ferrer Aledo, en el Catàlech dels peixos de l'illa de Menorca. (Observaciones, página 40), peró creyèm no pot relacionarse amb el Plectropoma fasciatum, Costa, ni amb la Cerna Chrysótaenia, Doderl., ni ésser una varietat del Serranus cabrilla, Linn., car totes elles, encara que tinguin la cúa partida, no més tenen $3 / 8$ ratjs en l'anal, essent així que aquesta especie'n té 3/12.

\section{Género, AnTHIAs. Anthias sacer, Bloch.}

Syn.: Aylopon Ivicae; A. hispanus; A. Rissoí; A. Nicensis; A. Algeriensis, Guichen.; Serranus anthias, Cuv. et Valenc., Guichen.; Avlopon anthias, Riss., Rafin., Guichen.; Lutjanus anthias, Lacep., Riss.; Anthias sacer, CBp., Lowe, Günth., Canestr.: Labrus anthias, Linn.

Nom vulgar: Rexet, Barcelona; Somera, Costa de Llevant; Dentó, Menorca.

Sub-famllia dels Apogonini.
Género, Apogon, Lacep.
Apogon imberbis, Günth.

Syn.: Apogon rex, Lowe; Apogon rex mullorum, Cuv, et Valenc., Günth.; Mullus imberbis o Apogon, Cuv.; Apogon ruber, Rafin., Lacep., Riss.; Perca pusilla, Del.; Centropomus rubens, Spinola: Mullus imberbis. Bonnat.; Mullus imberbis sive re.r mullorum, Willugh.; Corvulus, Gésner.

Nom vulgar; Rey, Reyet, Tarragona: Moll reyal, Anfós, BarceIona: Mare d'anfós, Muret vermell, Menorca. 


\section{Familia dels SCIENIDE Género, Umbrina, Cuv. Umbrina Cirrosa, Riss.}

Syn.: Umbrina,Yarr., Couch; Umbrina cirrhosa, CBp., Günth. Canestr.; Umbrina vulgaris, Cuv. et Valenc., Guichen.; Perca umbra, Lacep.; Sciena cirrhosa, Linn., Bloch. Agass.; Coracinus, Salvian.; Chromis, Bell, Gesner.

Nom vulgar: Corvall, Barcelona, Tarragona; Red, Reig, Me norça.

\section{Sciœna aquila, Cuv.}

Syn.: Scicena, Couch.; Sciœna umbra, CBp.; Sciona aquila, Günth., Canestr., H. Schlegel, Cuv. et Valenc., Guichen., Riss.; Perca Vanloo, Riss.; Cheilodiptera aquila, Lacep.; Umbrina, SaIvian.; Úmbra marina, Bell.

Nom vulgar: Retx, Tarragona.

Especie prou coneguda de tots els nostres pescaires, però no citada en cap Catàlech de peixos de Catalunya.

N'havèm vist exemplars pescats á l'art (28 d'Abril de 1910) de més de 30 kilograms de pes.

\section{Corvina nigra.}

Syn.: Corvina nigra, CBp., Canestr., Gunth., Cuv. et Valenc.; Sciona nigra, Bloch; Sciona umbra, Lacep., Riss., Linn., Rosenthal; Coracinus niger, Salvian., Willugh.; Coracinus, Rond.

Nom vulgar: Corva, Corvina, Tarragona; Corva, Barcelona; Escurbai, Menorca.

Género, Pristipoma, Cuv. Pristipoma Bennettii, Lowe.

Syn.: Pristipoma ronchus, Valenc., Guichen.; Pristipoma Bennettii, Valenc., Guichen., Günth., Steidachner, Rochebrune. .

Nom vulgar: Roncador, Tarragona.

Especie nova per Catalunya. El Roncador no es raro, car en 2 de Juliol de 1910 ne vejerem 12 de juntś á la pescatería de Tarragona, pescats á les xarxes, y son molts els dies que se'n agafen al palangró. 
Familia dels ScOMBRIDÆ.

Sub-familia dels Scombrini.

Género, Scomber.

Scomber Scomber, Linn.

Syn.: Scomber Scombrus, CBp., Lacep.; Scomber Scomber, Bonnat., Riss., Cuv. et Valenc., Brunn., Bloch., Günth., Schlegel, Canestr.

Nom vulgar: Barat o Vera, Barcelona, Tarragona, Menorca.

Abunda molt. $\bar{n} n$ una sola llevada, á vegades se'n pesquen més de 2.000 kilograms.

\section{Scomber Colias, Linn.}

Syn.: Scomber macrophthalmus, Rafin., CBp.; Scomber pneumatop.horus, CBp., Guichen., Cuv. et Valenc., Del.; Scomber Colias, Cuv. et Valenc., Riss., Gml., Günth., Canestr.; Colias, Rond., Lacep.

Nom vulgar: Cavalla, Bisst:, Barat d'ull gros, Tarragona; Barat o Verat, Barcelona, Menorca; Bissu, Costa de Llevant.

El Barat petit ó da cria es l'especie coneguda per:

\section{Scomber pneumatophorus, Delaroche.}

Nom vulgar: Gallimó, Tarragona.

Les especies Scomber colias, Linn.; Dentex macrophtalmus, C'uv. et Valenc., y C!prinus macrophtalmus, Lacep. (Long-TsingYu. Aquarium del Park de la Ciutadela de Barcelona) ofereixen certa hipertrofia ocular; y caldría estudiar si existeix reducció del nervi óptich. ¿Será tal volta efecte d'una adaptació especial, per passar o haver passat temporalment á viurer o habitar en un nou medi fosch, forçant per tant llur organ visual á fí d'aprofitar èls febles ratjs de llum que hi arriven, com succeheix als animals caverricols de la fosca?

\section{Género, Auxis.} Auxis Bisus, CBp.

Syn.: Aurris Rochei, Günth., Canestr.; Auris vulgaris, Cuv. et Valenc.: Thynnus Rocheunus, Riss.; Scomber Bisus, Rafin.; Bize, Duham. 
Nom vulgar: Melva, Barcelona, Tarragona; Baldufa, Costa de Llevant.

Quan se calaven peces surants a fonera per no existir aleshores navegació a vapor, se'n agafaven moltíssimes ınés que ara y avuy encara a la Torredembarra se'n pesquen bastantes, á la bolitxa.

\section{Género, THynnus. \\ Thynnus Thunnina, CBp.}

Syn.: Thynnus thunnina, Günth., Canestr., Cuv. et Valenc., Thvnnus Leachianus, Riss.: Scomber Commersonii, Riss.

Nom vulgar: Arbecona, Tarragona; Tunyina, Barcelona; Albe cora, Costa de Llevant; Bacoretes, Goles de l'Ebre.

Thỹnus thỵnus, Günth.

Syn.: Thynnus valgaris, CBp.: Canestr., Cuv, et Valenc., Guichen.; Thynnus mediterraneus, Riss.; Scomber thynnus, Lacep., Riss., Bonnat., Linn., Bloch, Brunn.; Thynnus, Duham., Bell.

Nom vulgar: Tunyina, Barcelona, Tarragona.

A les petites ó de cria, vulgarment se les coneix ab el nom de Golfás.

En l'almadraba del cap del terme, Hospitalet-Atmetlla (Tarragona), pêl Malg se'n pesquen moltes de 1 kilogram de pes y pêl Septembre escassament de mitj kilogram; aixó á part de la matansa que segons les anyades, com en l'almadraba de Rosas, se'n fà de molt més grosses: llevades de 200, 300 y 600 .

Tynnus alalonga, Cuv. et Valenc.

Syn.: Orcynus alalonga, Riss., CBp., Canestr., Günth.; Scomber alalonga, Riss., Bonnat; Scomber germo, Lacep.; Germon, Duham.; Alilangh, Duham.; Alalungha, Cetti.

Nom vulgar: Zanja, Goles de l'Ebre.

Especie molt rara, nova per Catalunya. Abunda més vers les aygues de Ponent y de l'Estret.

Género, Pelamys.

Pelamis sarda, Cuv, et Valenc.

Syn.: Pelamis sarda, Guichen., Willugh., CBp., Günth., Canestr.; Thynnus sardus, Riss.: Thynnus pelamis, Riss., Brunn.; 
Scomber mediterraneus, Del.; Scomber sarda, Lacep., Riss., Bloch, Rosenthal; Pelamis, Salvian., Bell.

Nom vulgar: Bonitu, Barcelona, Tarragona; Bonitol, Menorca.

A darrers d'Agost y á primers de Septembre se'n pesquen mol tíssims á l'art.

Sub-familia dels Carangini.

Género, Trachurus, Cuv.

Trachnrus Trachurus, Günth.

Syn.: Caranx trachurus, CBp., Schlegel, Lacep., Riss.; Trachurus, Canestr.; Scomber trachurus, Bonnat., Linn., Bloch, Brunn.; Trachurus, Rond., Bell., Salvian., Willugh., Gesner.

Nom vulgar: Surell, Surellól petit, Tarragona, Barcelona, Menorca.

Abunda molt. El Surell pescat al bou te $0440 \mathrm{~m} \cdot$ o $0 ‘ 50 \mathrm{~m}$. de llarch.

Género, Caranx, Cuv.

Caranx Fusus, Geof. St. Hil.

Syn.: Caran.r Fusus, Cuv. et Valenc., Günth.

Nom vulgar: Surell, Barcelona, Tarragona. Especie nova per Catalunya. Abundant y conegudíssima. En Amador Romani y Guerra, de Capellades, l'observá y classificá en 1909.

\section{Caran.x suareus, Riss.}

Syn.: Caran.x suareus, Cuv. et Valenc.

Nom vulgar: Surella, Tarragona.

Especie nova per Catalunya. En Febrer y Mars de 1911 se'n pescaren moltes en les aygues de la part occidental de Catalunya.

Sub-familia dels Centronotini.

Género, NAUCRATES, Rafin.

Naucrates ductor, Cuv. et Valenc.

Syn.: Naucrutes ductor, CBp., Günth., Canestr., Guichen.; Naucrates fanfarus, Ratin.; Centronotus conductor, Lacep., Riss.; Gasterosteus ductor, Bonnat., Linn., Brunn.; Scomber ductor, Bloch. 
Nom vulgar: Bairó, Tarragona; Pampol, Barcelona, Menorca; Biró, Costa de Llevant.

Aquest petit peix, guia y fidel company del tatró, segueix als barcos de navegació d'altura y al arrivar a port embesteix com es maperdut a les platjes y fins se deixa agafar amb les mans.

\section{Género, Lichia, Cuv.}

Lichia glaucus, Cuv. et Valenc.

Syn.: Lichia glauca, Günth., Canestr.; Lichia glaucus, CBp., Lichia glaycos, Riss.; Centronotus glaycos, Riss.; Caran.x glaucus, Lacep.; Glauctis (Derbio), Rond.

Nom vulgar: Palomida, Tarragona; Columida, Barcelona; Palo mida, Palomina, Menorca.

\section{Lichia amia, Cuv. et Valenc.}

Syn.: Lichia amia, Guichen., Agass., CBp., Günth., Canestr.; Lichialvzan, Lacep., Riss.; Centronotus vadigo, Lacep.; Caran.r amia, Lacep.; Scomber amia, Linn.; Amia, Salvian.; Lampuga, Bell.

Nom vulgar: Palomida, Tarragona; Colomida, Barcelona; Palomida, Surell de penya, Menorca.

Lichia vadigo, Cuv, et Valenc.

Syn.: Centronotus vadigo, Riss.; Lichia vadigo, Riss., CBp., Günth., Canestr.; Centronotus glaycos, Lacep.

Nom vulgar: Lexola, Tarragona; Lexa, Costa de Llevant. Especie no citada en cap Catálech de peixos de Catalunya. A vegades se'n agafen moltes a palangra.

Género, Seriola, Cuv. Seriola Dumerilii, Riss.

Syn.: Seriola Dumerilii, Günth., CBp., Canestr., Cuv. et Valenc., Guichen.; Caran.x Dumerilii, Riss.

Nom vulgar: Verderol, Tarragona; Sirvia, Sirviola, Verderol, Menorca. 
Género, Temnodon, Cuv. et Valenc.

Temnodon saltator, Cuv.

Syn.: Scomber plumbeus, Mitchill; Gasterosteus saltatrix, Linn., Bonnat.: Pomatomus, Skib, Lacep.; ('heilodipterus heptacanthus, Lacep.: Saltatri.r, Catesby.; Temnodon saltator, Valenc., S. Berthel., Guichen., Nordm., CBp., Günth., Verany., Steindachn., Canestr., Brit. Capel., Giglion., Perugia.

Nom vulgar: Tsernia, Tarragona. Especie nova per Catalunya. En pochs dies de diferencia (Agost 1910) en la pescatería de Tarragona, ne vegerem dos exemplars pescats a les xarxes, que pesaven quiscun 15 kilogram.

Sub-familia dels Zeini.

Género, ZEus, Arted.

Zeus faber, Linn.

Syn.: Zeus faber, Bloch, Rosenthal, Agass., CBp., Günth., Schlel., Canestr., Bonnat., Lacep., Riss., Cuv. et Valenc., Guichen.

Nom vulgar: Gall, Barcelona, Tarragóna; Gall de St. Pere, Menorca.

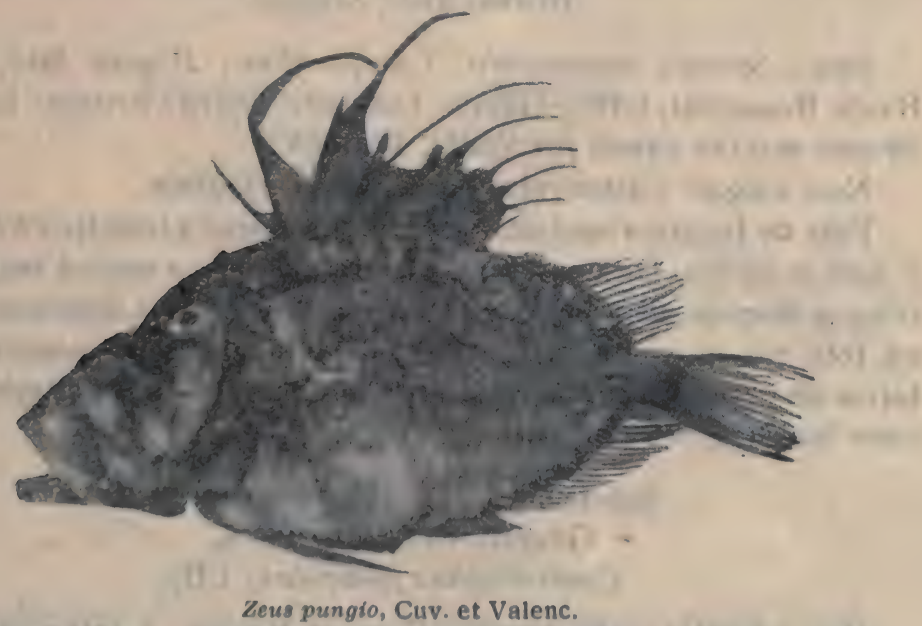

Zens pungio, Cuv, et Valenc.

Syn.: Zeus pungio, CBp., Günth., Guichen.

Nom vulgar: Gall, Tarragona. Especie nova per Catalunya. Pes cat a l'art, en 10 de Juny de 1911, n'adquirirem un bell exemplar. 
Sub-familia dels Caprini.

Género, CAPros, Lacep.

Capros aper, Lacep.

Syn.: Capros aper, CBp., Gürth., Canestr.; Riss., Guichen.; Perca pusilla, Brunn.; Zeus aper, Linn.; Aper Rondeletii, Willugh.

Nom vulgar: Gallet, Barcelona; Xavo, Tarragona.

Sub-familia dels LAMPRINI.

Género, LAMPRIS, Retzius.

Lampris guttatus, Retzius.

Syn.: Lampris luna, Günth., Canestr.; Lampris lauta, Lowe; Chrysotosus Luna, Lacep., Riss.; Lampris guttatus, Cuv. et Valenc., CBp., Schleg.

Nom vulgar: Llampuga, Tarragona. Especie rara y nova per Catalunya. Abunda en aygues de les Mallorques.

Sub-familia dels BRAMIN.

Género, Brama. schneid.

Brama Raii, Schneid.

Syn.: Sparus castaneola, Lacep., Riss.; Brama Raii, Riss., Bloch, Rosenthal, CBp., Günth., Canestr.: Sparus brama, Bounat.; Brama marina cauda forcipata, Willugh.

Nom vulgar: Castanyola, Barcelona, Tarragona.

Peix de fonera abundant, que te de pescarse a fons fixo d'aygues, ni mes ni menys. Desapareix o emigra una llarga série d'anyades y retorna després altra vegada. Dintre la seva massa muscular, quas en tots ells hi viu un tricocefal tal volta nou: verm nematodes en forma de petita carabassa vinatera, llarch de $0^{\circ} 07 \mathrm{~m}$., glutinós y de color blanch-groch de canari.

\section{Sub-familia dels CEnTrolophinI \\ Género, Centrolophus. \\ Centrolophas pompilus, CBp.}

Syn.: Centrolophus morio, Cuv. et Valenc.; Centrolophus ni. ger, CBp., Lacep.; Centrolophus pompilus, Günth., Canestr., Guichen., Cuv. et Valenc.; Centrolophus pompilius, Riss.; Pompilus, Couch, Rond.; Coryphæna pompilus, Lacep., Riss., Linn.; Serrá de Provença, Duham. 
Nom vulgar: Trotllu, peix de brom, Barcelona; Negret, Negritu. Tarragona.

S' $n$ agafen pel Mars y Abril barrejats entre les moles de surell.

Género, Stromateus.

Stromateus Fiatola, Linn.

Syn.: Chrysostomus fiatoloides, Lacep.; Stromateus fiatola, Lacep., Riss., Cuv. et Valenc., Guichen., Bonnat., CBp., Günth., Canestr.; Hepatus, Gesner; Stromateus, Rond., Gesner, Aldrov., Willugh.; Fiatola, Rond., Gesner; Callychthys, Bell.

Nom vulgar: Palomida, Barcelona, Tarragona.

Stromatells Microchirus, Günth.

Syn.: Fietola fasciata, Riss.; Seserinus microchirus, Cuv. et Valenc.: Seserinus Rondeletii, Cuv. et Valenc., Cuv.; Stromateus microchirus, CBp., Canestr.; Centrolophus microchirus, Bonelli; Seserinus, Rond., Gesner.

Nom vulgar: Pampol pudent, Tarragona. Especie nova per Catalunya.

\section{Sub-familia dels CORYPHeninI. \\ Género, CoRyphena. \\ Corypheena hippurus, Risś.}

Syn.: Coryphœna pelágica, CBp., Günth., Canestr., Riss.; Lampugus pelagicus,, Cuv. et Valenc.; Coryphona hippurus, Lacep., Riss., Cuv. et Valenc., Guichen., Linn., Bloch, CBp., Günth., Canestr.; Equiselis, Rond.

Nom vulgar: Doradu, Tarragona; Llempuga, Menorca.

$$
\begin{gathered}
\text { Sub-familia dels Xipheini. } \\
\text { Género XIPHIAS. } \\
\text { Xiphias gladius, Linn. }
\end{gathered}
$$

Syn.: Xiphias gladius, Cuv. et Valenc., Guichen., Lacep., Bloch, Rosenthal, CBp., Günth., Schleg., Canestr.; Xiphias piscis, WiIlugh.; Xiphius, Bell.; Gladius, Salvian.

Nom vulgar: Peix espasa, Tarragona; Emperador, peix espasa, Menorca. 
Sub-familia dels Echeneini.

Género, Echeneis, Arted.

Echeneis remora, Linn.

Syn.: f'emora, Couch., Yarr., Rond., Aldrov.; Echeneis remora, Lacep., Riss.. Bonnat, Bloch, Rafin., Cuv., Rosenthal, Costa, CBp., Günth., Canestr.

Nom vulgar: Pegas, Tarragona; Rémora, Barcelona.

Havem vist qualques exemplars momificats procedents de Ia Torredembarra.

Familia dels Trichiuridae.

Género, Lepidopus, Goüan.

Lepidopus argenteus, Bonnat.

Syn.: Lepinopus caudatus, Günth., Canestr.; Lepidopus ensiformis, CBp.; Lepidopus, Agass, Lepidopus argyreus, Cuv. et Valenc., Guichen.: Lepidopus guanianus, Lacep., Riss.; Lepidopus Peronii, Riss.; Lepidopus argenteus, Bonnat.; Trichiurus caudaIus, Euphras., Arted.; Lepidopus, Goüan.

Nom vulgar: Serra, Serreta, Tarragona. Especie nova per Catatunya. En 24 de Mars de 1910 n'adquirirem un hermós exemplar pescat a palangre de més de 2 metros de llarch.

Familia dels Tanioida.

Sub-familia dels Cepolini.

Género, Cepola, Linn.

Cepola Rubescens, Linn.

Syn.: Cepola Rubescens, Cuv. et Valenc., Guichen., Riss., Brunn., CBp., Günth., Canestr.; Cepola serpentiformis, Lacep.; Cepola tonia, Bloch, Riss.; Trenia rubra, Willugh.; Myrus alter sive serpens rubescens, Rond., Aldrov.

Nom vu!gar: Pixota vermella, Llígas-cames, Tarragona; Beta, Barcelona; Látigo, Costa de Llevant; Flámula, Menorca.

Género, Regalecus, Brunn.

Regalecus gladius, Günth.

Sym.: Gymnetrus longiradiatus, Riss.; Gymnetrus gladius, Cuv. et Valenc.; Regalecus gladius, Canestr.

Nom vulgar: No'n té. Especie molt rara y nova per Catalunya. No fa massa temps, en Lluis Soler y Pujol, naturalista preparador de 
Barcelona 'n va tenir un exemplar pescat en aygues de Vilanova y Geltrú.

$$
\begin{aligned}
& \text { Género, Trachypterus, Goüan. } \\
& \text { Trachipterus Falx, Cuv. et Valenc. }
\end{aligned}
$$

Syn.: Truchypterus tceniu, Costa., CBp., Günth., Canestr.; G!mnetrus cepedianus, Riss.; Ful.x, Bell.

Nom vulgar: Fleuma, Menorca. Especie rara.

El 20 de Desembre de 1912 ne vegerem un exemplar d"1'40 m., pescat a palangre, que l'adquirí un capitá de la marina mercant.

\section{Trachypterus leiopterus, Günth.}

Syn.. Truchypterus leiopterus, Cuv. et Valenc.

Nom vulgar: Faixa, Tarragona. Rara.

El 15 de Desembre de 1911 n'examinarem un exemplar pescat a palangre, fẹt malbé al desenferirio y bastant descompost, quals proporcions eren: Iları̧raria total. $1 ‘ 50 \mathrm{~m}$., alçaria $0 ` 145 \mathrm{~m}$., doblaria 0,012 metros.

\section{Trachypterus cristatus, Bonelli.}

Syn.: Trachypterus cristatus, Günth.; ;Trachvpterus Bonell, Cuv. et Valenc.

Nom vulgar: Cardenal, Tarragona. Especie rara.

Familia dels Sparida. Sub-familia dels Sargini, Género, Sargus, Cuv. Sargus vulgaris, Geof. St. Hil.

Syn.: Sargus vulgaris, Günth., Brit.; Sargus Salviani, CBp., Canestr., Cuv. et Valenc., Guichen.; Sargus puntazzo, Riss.; Sparus sargus, Brunn.; Sargus, Bell.

Nom vulgar: Variada, Tarragona, Menorca; Sarg, Barcelona; Barada, Costa de Llevant,

\section{Sargus Rondeletii, Cuv, et Valenc.}

Syn.: Sargus sargus, Riss ; Sparus sargus, Riss.; Sargus Rondeletii, CBp., Günth., Canestr.; Sargus raucus, Geof. St. Hil.; Sparus varialus, Bonnat., Lacep.; Sparus, Bell.

Nom vulgar: Sard, Tarragona, Menorca; Sarg, Barcelona. 
Sargus velula, CBp.

Syn.: Sargus vetula, Günth., Cuv. et Valenc., Guichen.; Scarus onias Rondeletii, Willugh.; Scarus, Rond.

Nom vulgar: Sard, Tarragona. Especie nova per ${ }_{\mathrm{a}}$ Catalunya. Rara.

Sargus annularis, Geof. St. Hil.

Syn.: Sargus annularis, CBp., Günth., Canestr., Guichen., Cuv. et Valenc.; Aurata annularis, Riss.; Sparus haffara, Riss.; Sparus sparalus, Lacep.; Sparus smaris, Brunn.; Sparus annularis, Linn., Del.

Nom vulgar: Esparrall, Esparralló, Pinta, Tarragona; Esparrall, Esparrai, Barcelona, Menorca.

\section{Género, Charax, Riss.}

Chara.x Puntazzo, Cuv, et Valenc.

Syn.: Charax acutirostris, Riss.; Sparus puntazzo, Riss.; Spa. rus acutirostris, Del.; Charax puntazzo, Guichen., CBp., Günth., Canestr.; Puntazzo, Celti.; Sparus, Aldrov.

Nom vulgar: Morruda, Tarragona, Menorca; Auradeta, Barcelona.

\section{Sub-familia dels Obladini. \\ Género, Bóx, CBp. Box Boops, Cuv.}

Syn.: Boxvulgaris, Günth., Capel., Cuv. et Valenc., Guichen.; Box boops, Canestr.; Boops vulgaris, Riss.; Sparus boops, Lacep., Riss., Bonnat., Linn., Brunn., Rafin.; Boga, Duham., Rond.; Boops Rondeletii primus, Willugh.; Boces vel Boopes, Bell.

Nom vulgar: Boga, Bogarró, Barcelona, Tarragona, Menorca; Molt abundant.

Aquest peix, la Mabre (Pagellus mormyrus, Linn.) y 'Is Torts (Labrus y Crenilabrus) son els mes atacats pel crustaci Nerocila bivittata, Edv. anomenat poll de mar.

\section{Box salpa, Cuv, et Valenc.}

Syn.: Boops salpa, Riss.; Sparus salpa, Linn., Bloch, Brunn., Rafin., Lacep., Riss.; Bo.x salpa, Guichen., CBp., Günth., Canestr.; Salpa, Bell., Salvian., Willugh., Rond., Duham. 
Nom vulgar: Salpa, Barcelona, Tarragona, Menorca.

Aquesta especie s'alimenta quasi exclusivament d'herba cancrera (Ulva lactuca. Linn.), alga marina verda de la familia de les Confervaceas.

Género, Oblada.

Oblada melanura, BCp.

Syn.: Boops melanurus, Riss.: Oblada melanura, Cuv. et Valenc., Guichen., Günth., Canestr.; Sparus melanurus, Riss., Linn., Brunn., Rafin.; Sparus Oblada, Lacep.: Oblada, Duham.; Melanurus, Bell.

Nom vulgar: Oblada, Barcelona, Tarragona, Menorca.

Sub-familia dels Sparini.

Género, Pagellus, Cuv.

Pagellus erịthrinus, Cuv. et Valenc.

Syn.: Erythrinns, Couch.; Pagellus erythrinus, Guichen., CBp., Günth., Canestr.; Pagrus erythrinus, Riss.; Sparus erythrinus, Lacep., Riss., Bonnat., Bloch, Brunn.

Nom vulgar: Pagell, Barcelona, Tarragona; Pitjell, Menorca.

Cinquanta anys enrera, avans de generalisarse la pesca á l'arrastre y començarse á no respectar les vedes y les lleys de pesca, aquesta especie abundave moltíssim mes; amb l'arrastre s'han destruit les pastures y tot plegat ha portat la crisis perpétua en que viu Ia classe pescadora.

En el sigle XIV el prelat tarragoní, l'infarit en Joan d'Aragó, obtingué del seu germá 'l comte de Prades que no exigís als pescadors de Tarragona que anaven á pescar vers la costa del coll de Balaguer el tribut de 50 peixells ó llur válua en diner en concepte de dret de riberatge.

\section{Pagellus hreviceps, CBp.}

Syn.: Pagellus hreviceps, Guichen.. Cuv, et Valenc.

Nom vulgar: Pixano, Tarrragona; Pitjell, Menorca.

Aquest peixet y'l Chromis castanea, Cuv., son les especies que animen mes les encalmades aygues de nostres cales y ports.

Pagellus Bogaraveo, CBp.

Syn.: Pagrus Bugaravella, Riss.: Pagellus hogaraveo, Günth., 
Guichen., Canestr., Cuv. et Valenc.; Sparus bogaraveo, Lacep., Riss., Bonnat., Brunn.; Pilonneau ó Lagadec, Duham.; Bogue Ravel, Rond.

Nom vugar: Bogaravell, Tarragona, Menorca.

\section{Pagellus mormvrus, Linn.}

Syn.: Pagrus morm!rus, Geof. St. Hil., Riss.; Pagellus mormyrus, Cuv. et Valenc., Guichen., Günth., Canestr.; Sparus mormyrus, Riss., Lacep., CBp., Brunn., Rafin.; Morme, Rond., Duham., Bonnat.; Mormyrus, Bell., Salvian.

Nom vulgar: Mabre, Barcelona, Tarragona, Menorca.

$$
\text { Pagellus acarne, CBp. }
$$

Syn.: Pagrus acarne, Riss.; Pagellus acarne, Günth.. Guichen., Canestr., Cuv. et Valenc.; Sparus herda, Riss.; Acarne, Rond.

Nom vulgar: Quelet, Tarragona; Besuch, Barcelona, Menorca.

Avans abundave moltíssim, mes en nostres dies sembla haverse retirat de les aygues de la Catalunya occidental vers les de Barcelona y de llevaint.

El Pagellus centrodontus, CBp. (Besugo) que tots els díes se ven en nostres mercats, procedeix del mar Cantábrich.

$$
\begin{gathered}
\text { Género, Pagrus. } \\
\text { Pagrus vulgaris, Cuv. et Valenc. }
\end{gathered}
$$

Syn.: Sparus argenteus, Del.: Pagrus argenteus, Riss.; Spagrus pagrus, Lacep., Riss., Linn., Rafin.; Pagrus vulgaris, Guichen., CBp., Günth., Canestr.; Pagrus, Rond.

Nom vulgar: Pagre, Barcelona, Tarragona, Menorca; Pargo, Pargoti, Goles de l'Ebre.

$$
\text { Pagrus Orphus, CBp. }
$$

Sẏn.: Auratu Orphus, Riss.; Sparus pagrus, Brunn.; Pagrus Orphus, Cuv. et Valenc., Gûnth.

Nom vulgar: Pagre, Tarragona, Menorca; Pargo, Pargotí, Goles de l'Ebre. Especie rara.

Género, CHRYSOPHRIS.

Chrysophris aurata, Günth.

S yn.: Aurata semilunata, Riss.; Sbarus aurata, Lacep., R iso 
Linn., Bloch, Brunn., CBp.; Chrı̣sophris aurata, Canestr., Cuv. et Valenc., Guichen.; Aurata, Bell.. Salvian., Willugh.

Nom vulgar: Orada ó Aurada, Auradella, Barcelona, Tarragona, Menorca: Moxarra, Goles de l'Ebre.

\section{Chrysophris Crassirostris, Günth.}

Syn.: Sparus Crassirostris, CBp.: Chr!'sophris Crassirostris, Cuv. et Valenc.

Nom vulgar: Orada ó Aurada, Auradella, Tarragona, Menorca; Moxarra, Goles de l'Ebre.

\section{Sub-familia dels Cantharini. \\ Género, Cantharus, Cuv. \\ Cantharus griseus, Cuv, et Valenc.}

Syn.: Cantharus lineatus, Günth., Canestr.; Cantharus vulgaris, CBp., Cuv, et Valenc., Guichen.; Sparus lineatus, Montagu; Cantharus tanuda. Riss.: Sparus Cantharus, Lacep., Linn., Riss.; Cantheno, Rond.

Nom vulgar: Cántara, Barcelona, Tarragona, Costa de Elevant, Menorca.

\section{Cantharus Brama, Cuvi et Valenc.}

Syn.: Cantharus hrama, CBp., Günth., Guichen.

Nom vulgar: Cántara, Tarragona. Especie nova per Catalunya. Rara.

Cantharis Orbicnlaris, Cuv, et Valenc.

Syn.: Cantharus orbicularis, CBp., Günth.. Canestr.

Nom vulgar: Xopa, Tarragona; Càntara. Menorca.

Sub-familia dels Denticini.

Género, Dentex, Cuv. Dentex vulgario, Cuv.

Syn.: Sparus denlex, Lacep., Riss., Linn.. Brunn., Rafin.; Dentexrulgaris, CBp., Günth., Brit, Capel., Canestr., Riss., Cuv. et Valenc., Guichen.: Dentex, Salvian., Couch, Rond.: S!nugris, Bell.

Nom vulgar: Dento, Tarragona; Déntol, Barcelona, Menorca. 
Dentex Macrophthalmus, Cuv. et Valenc.

Syn.: Dentex erithrostoma, Riss.; Dentex macrophthalmus, CBp., Günth., Brit. Capel., Canestr., Guichen.; Sparus macropht. halmus, Lacep., Riss., Bloch; Dentalis ó Dente.x, Bell.

Nom vulgar: Pagell Dento, Tarragona; Dentol, Barcelona, Menorca. Especie molt rara á la qual cal també aplicar lo que havem dit referent á las especies: Scomber Colias, Linn. y Cvprinus macrophthalmus, Linn.

Dentex Synodon, Riss.

Syn.: Sparus gibbosus, Rafin.; Dentex gıbbosus, Cocco.

Nom vulgar: Corcovada, Tarragona. Especie nova per Catalunya. Molt rara; abunda més, vers les aygues de Ponent y de l'Estret.

Familia dels Monido.

Género, Mana, Cuv.

Mona vulgaris, Cuv, et Valenc.

Syn.: Sparus mœna, Linn., Brunn., Lacep., Riss.; Smaris mœna, Riss.; Mona vulgaris, Guichen., CBp., Günth., Canestr.

Nom vulgar: Mata soldats, Xucla vermella, Tarragona; Xucla d'Hivern, Menorca.

\section{Mcena Osbeckii, Cuv, et Valenc.}

Syn.: Moena zebra, Günth.; Mona Osbeckii, Guichen., CBp., Canestr.; Sparus tricuspidatus, Max. Spinola: Smaris gora, Riss.; Sparus Osbeckii, Riss.

Nom vulgar: Xucla guindana, Xucla d'Estiu, Tarragona: Xucla, Barcelona; Mora, Menorca.

\section{Mona Jusculum, Cuv. et Valenc.}

Syn.: Mcena Jusculum, CBp., Canestr.; Mendole, Rond.

Nom vulgar: Mata soldats, Xucla d'Hivern, Tarragona; Xucla, Barcelona, Menorca.

A les peces, el 15 de Juliol de 1911 , se'n pescaren á Tarragona més de 150 kilograms. 
Mona vomerina, Cuv. et Valenc.

Syn.: Mona vomerina, CBp., Günth.

Nom vulgar: Xucla vera, Barcelona, Tarragona.

Género, Smaris, Cuv.

Smaris vulgaris, Cuv, et Valenc.

Syn.: Mcena smaris, Canestr.; Smaris vulgaris, CBp.

Nom vulgar: Jerret, Barcelona, Tarragona, Menorca.

Smaris Alcedo, Cuv, et Valenc.

Syn.: Moena alcedo, Canestr.; Smaris smaris, Riss.; Sparus alcedo, Riss.; Smaris alcedo, CBp., Günth., Guichen.

Nom vulgar: Jerret, Tarragona; Jerret, Mata soldats, Barcelona; jerret mascle, Menorca.

\section{Smaris Maurii, CBp.}

Syn.: Mona gracilis, Canestr.; Mona Maurii, Canestr.; Smaris Maurii, Günth.; Smaris gracilis, CBp., Günth.; Sparıs smaris, Del.

Nom vulgar: Jerret, Tarragona; Jerret de Sant Pere, Menorca. Especie rara.

Smaris Chryselis, Cuv. et Valenc.

Syn.: Smaris gagarella, Cuv. €t Valenc., CBp.; Smaris Chryselis, CBp.

Nom vulgar: Jerret, Tarragona; Xucla blanca, Barcelona.

Smaris insidiator, Cuv, et Valenc.

Syn.: Mana insidiator, Canestr.; Smaris insidiator, CBp., Günth., Brit. Capel.

Nom vulgar: Jerret, Tarragona; Jerret inglés, Menorca.

Familia dels Labrido.

Género, LABRUS, Arted.

Labrus Turdus, Linn.

Syn.: Labrus turdus, Lacep., Riss., Cuv. et Valenc., Guichen., $\mathrm{CBp}$, Canestr. 
58 Fauna ictiológica de Catalunya

Nom vulgar: Tort vert, Tarragona, Barcelona; Massot, Me norca.

$$
\text { Labrus merula, Linn. }
$$

Syn.: Labrus caruleus, Riss,; Labrus Libens, Brunn.: Labrus merula, Lacep., Riss., Cuv, et Valenc., CBp., Günth., Canestr.; Turdus niger, Willugh.; Merula, Salvian., Aldrov.

Nom vulgar: Tort negre, Barcelona, Tarragona; Tort massot, Menorca.

Var: Labrus lividus, Cuv. et Valenc,

Nom vulgar: Tort negre, Tarragona. Varietat nova per Catalunya.

Var. Labrus limbatus, Cuv, et Valenc.

Nom vulgar: Tort, Tarragona; Tort vert, Griva, Barcelona.

$$
\text { Labrus lineolatus, Cuv. et Valenc. }
$$

Syn.: Labrus lineolatus, Canestr.

Nom vulgar: Tort, Tarragona; Massot, Menorca.

$$
\text { Labrus festivus, Riss. }
$$

Syn.: Labrus turdus, Brunn.; Labrus festivus, CBp., Günth., Canestr., Cuv. et Valenc.; Turdus oblongus fuscus maculosus, Willugh.

Nom vulgar: Grivia, Tarragona, Menorca.

$$
\text { Labrus luscus, Linn. }
$$

Syn.: Labrus luscus, Lacep., Riss., Cuv. et Valenc.

Nom vulgar: Grivia, Tarragona; Massot, Menorca.

$$
\text { Labrus viridis, Linn. }
$$

Syn.: Labrus turdus, Riss.; Labrus psittacus, Lacep.: Labrus viridis, Cuv. et Valenc., Riss., CBp.; Turdus viridis major, Willugh.

Nom vulgar: Grivia, Tarragona; Massot, Menorca. 


\section{Var. Labrus nereats, Riss.}

Syn:: Labrus nereus, Cuv. et Valenc.

Nom vulgar: Grivia. Tarragona. Especie nova per Catalunya.

$$
\text { Labrus mixtus, Linn. }
$$

(Mascle)

Syn.: Labrus variegatus, Lacep., Riss.; Labrus vetula, Bloch; Ĺabrus ceruleus, Bonnat.; Labrus lineatus, Bonnat., Lacep., Riss.; Labrus mixtus; Cuv. et Valenc., Guichen., CBp., Günth., Canestr.; Turdus major varius prnecedenti similis, Willugh.; Turdus perhelle pictus, Willugh.

\section{(Femella)}

Labrus quadrimaculatus, Cuv. et Valenc., Guichen.; Labrus trimaculatus, Lacep., Riss., Bonnat., Pennant, Donovan; Labrus carneus, Ascanius, Bloch, CBp.; Labrus mixtus, Fries et Ekström.

Nom vulgar: Grivia, Tarragona; Tort blanch, Barcelona: Bestenaga, Menorca.

En 20 de Maig de 1910, adquirirem un may vist y bellíssim exemplar: era un mascle que midia $0.30 \mathrm{~m}$. de llarch y tenía un vistós color de salmó o ataronjat amb el cap, la cua y'l comensament de les aletes dorsal y anal y les puntes de les ventrals d'un blau celest puríssim.

Labrus sa.rorum, Cuv, et Valenc.

Syn.: Labrus sa.rorum, CB́p.

Nom vuigar: Tort, Tarragona; UIl de perdiu, Menorca.

Género, Crenilabrus, Cuv. Crenilabrus ocellatus, Nomann.

Syn : Lubrus ocellatus, Forskal, Bonnat.: Lutjanus ocellatus, Riss., Lacep.; Crenilahrus ocellutus, Riss., Cuv. et Valenc., Guichen., CBp., Günth., Canestr.

Nom vulgar: Tort, Barcel ona, Tarragona; Roquer, Ruqué, norca. 


\section{Crenilabrus Roissali, Riss.}

Syn.: Labrus, Brunn.; Lutjanus Roissali, Riss.; Lutjanus varius, L. Alberti, Riss.; Crenilabrus Roissali, Cr. varius, Riss.; Crenilabrus Roissalii, Cuv. et Valenc., CBp., Canestr.

Nom vulgar: Tort, Barcelona, Tarragona; Ruqué, Menorca.

Var. Crenilabrus quinquemaculatus, Riss.

Syn.: Crenilabrus quinquemaculatus, Günth., Cuv. et Valenc. Nonı vulgar: Tort, Tarragona; Temburé, Menorca.

\section{Crenilabrus tigrinus, Riss.}

Syn.: Labrus æruginosus, Nordmann.

Nom vulgar: Tort, Tarragona; Ruqué, Menorca.

\section{Crenilabrus melops, Riss.}

Syn.: Labrus melops, Linn., Bonnat., Lacep.; Crenilabrus melops, C Bp., Günth., Cuv. et Valenc., Guichen.; Crenilabrus Donovani, Cuv. et Valenc., Guichen.; Crenilabrus Couchii, Cuv. et Valenc.; Crenilabrus noruegicus, Cuv. et Valenc.; Lutjanus melops, Riss.; Labrus cornubius, Donov.; Lutjanus noruegicus, Lacep., Bloch.

Nom vulgar: Tort, Barcelona, Tarragona.

\section{Crenilabrus melanocercus, Riss.}

Syn.: Lutjanus melanocer cus, Riss.; Crenilabrus melanocercus, CBp., Günth., Canestr., Cuv. et Valenc.

Nom vulgar: Tort, Llambrega, Barcelona; Tort, Tarragona.

Crenilabrus Cœruleus, Riss.

Syn.: Crenilabrus coeruleus, CBp., Cuv. et Valenc., Günth.

Nom vulgar: Tort, Tarragona. Especie nova per Catalunya.

Crenilabrus Bailloni, Valenc.

Syn.: Crenilabrus Bailloni, CBp., Günth., Brit. Capel., Cuv. at Valenc.

Nom vulgar: Tort, Tarragona; Ruqué, Menorca. 
En Moreau no cita aquesta especie com habitante del Mediterrá, pero abunda bastant en les aygues de Tarragona.

\section{Crenilabrus mediterraneus, $\mathrm{CBp}$.}

Syn.: Perca mediterranea, Linn.; Lutjanus mediterraneus, Lacep., Riss.; Crenilabrus mediterraneus, Riss., Cuv, et Valenc., Guichen., Günth., Canestr.

Nom vulgar: Tort, Canari, Barcelona; Tort, Tarragona; Tert ruqué. Menorca.

\section{Var. Crenilabrus Boryanus, Riss.}

Syn.: C'renilabrus nigrescens, Riss.; Crenilabrus boryanus, Cuv. et Valenc.; Crenilabrus Pittima, CBp.

Nom vulgar: Tort, Mariquita, Barcelona; Tort, Tarragona.

Var. Crenilabrus Brunnichii, Riss.

Syn.: Lutjanus bidens, Bloch; Labrus Serpentinus, Bonnat.; Lutjanus Brunnichii, Lacep., Riss.; Crenilabrus Brunnichii, Cuv . et Valenc., CBp.; Labri, especies obscuriores, Brunni.

Nom vulgar: Tort, Barcelona, Tarragona.

\section{Crenilabrus Tinca, CBp.}

Syn.: Labrus tinca, Brunn.; Lutjanus tinca, Riss.; Lutjanus Cotta, Riss.; Crenilabrus Cotta, Riss.; Crenilabrus tinca, Riss., Cuv. et Valenc., Guichen., Günth., Canestr.

Nom vulgar: Tort, Barcelona, Tarragona; Satx, Ruqué, Menorca.

Crenilabrus arcuatus, Riss.

Syn.: Crenilabrus arcuatus, CBp., Cuv. et Valenc.

Nom vulgar: Tort, Tarragona. Especie nova per Catalunya.

Crenilabrus Chlorosochrus, Riss.

Syn.: Lutjanus Chlorosochrus, Riss.; Crenilabrus Chlorosochrus, CBp., Cuv. et Valenc.

Nom vulgar: Mariquita, Tort, Barcelona; Tort, Tarragona.

$$
\text { Crenilabrus pavo, } \mathrm{CBp} \text {. }
$$

Syn.: Pavo, Silvian.; Labrus pavo, Brunn., Bonnat., Lacep.; 
Lutjanus Geofroyius, Riss.; Lutjunus lapina, Riss.; Crenilabrus lapina, Cr. Geoffroi, Riss.; Crenilabrus pavo, Cuv. et Valenc., Guichen., Günth., Canestr.

Nom vulgar: Tort, Barcelona, Tarragona; Llabió, Costa de Llevant; Satx, Menorcá.

\section{Crenilabrus Massa, Riss.}

Syn.: Crenilabrus griseus, Günth., Canestr.; Lutjanus massa, Riss.; Labrus, Brunn.; Lutjanus cinereus, Riss.; Lutjanus cornubicus. Riss.; Crentabrus massa, Cr. Cotæ., CBp.; Crenilabrus cornubicus, Riss.; Crenilabrus massa. Cuv. et Valenc.; Crenilabrus Cottce, Cuv. et Valenc.

Nom vulgar: Tort, Barcelona. Tarragona; Temburé, Menorca.

Eis Lábrits, lo mateis que la Boga (Box Boops, Bp.) y la Mabra (Pagellus mormyrus, Linn.) son els peixos mes perseguits pel poll de mar, Nerocila bivittata Edv. que arriva a menjarsels les aletes y á foradarlos la pell, posant al descubert llur capa muscular.

Els pescayres crethen que qualques Torts son hibrits de Llobarro y de Grivia.

$\mathrm{Ab}$ un atent estudi podrien establirse molt més varietats no indicades en les obres.

Género, Coricus, Cuv.

Coricus rostratus, Nordmann,

Syn.: Lutjanus rostratus, Bloch, Lacep.; Lutjanus virescens, Riss.; Lutjanus Lamarchii, Riss.; Coricus virescens, C. Lamarckii, C. rubescens, Riss.; Coricus virescens, CBp.; Coricus rostratus, Cuv. et Valenc., Guichen., CBp.; Crenilabrus rostratus, Günth., Canestr.

Nom vulgar: Grivieta, Tarragona; Donzelleta, Barcelona; Petarch, Costa de Llevant: Trujeta, Menorca.

El Coricus ofereix les coloracions: blava, verda, roija y variada, lo que ha fet pensar a molts si serien especies distintes.

Género, Ctenolabrus, Valenc.

Ctenolabrus rupestris, Cuv, et Valenc.

Syn.: Labrus rupestris, Linn., Arted., Fries et Ekström.; Lut- 
ianus rupestris, Bloch; Ctenolabrus rupestris, Guichen., CBp., Günth.

Nom vulgar: Gripau. Barcelona. Especie rara. C'tenolabrus Iris, CBp.

Syn.: Ctenolubris iris, Cuv. et Valenc., Günth., Canestr.

Nom vulgar: Gripau, Barcelona.

N'havém vist de $0 ، 30 \mathrm{~m}$. de llarch amb tres taques negres: una al comensament dels ratjs molls de la dorsal; altra al final de la mateixa y la tercera damunt la base de la cua. I ensemps amb tretze ratjs molls en la dorsal y onze en l'anal.

Género, Acantholabrus, Valenc. Acantholabrus Palloni, Cuv, et Valenc.

Syn.: Crenilabrus exoletus, Riss.; Lutjanus Palloni, Riss.; Acantholubrus Palloni, CBp., Günth.. Canestr.

Nom vulgar: Tae rocas, Menorca.

En 16 de Juny de 1910 a la pescataría de Tarragona ne vegerem moltíssims exemplars pescats a l'artet.

\section{Género, Julis, Cuv.}

Julis vulgaris, Cuv, et Valenc.

Syn:: Coris Julis, Günth.: Julis mediterraneus, CBp., Riss.; Julis vulguris, Guichen., Canestr.; Labrus Julis, Bonnat., Lacep., Riss., Linn., Brunn., Bloch; Julis, Bell., Salvian.

Nom vulgar: Donzella, Tarragona, Menorca; Senyoreta, Donzella, Barcelona: Senyoreta, Güiula, Costa de Llevant.

Var: Julis speciosa, Riss.

Syn.: Julis speciosa, Cuv. et Valenc., Guichen.

Nom vulgar: Donzelia, Tarragona, Menorca; Senyoreta, DonzeIla, Barcelona Senyoreta, Güitula, Costa de Llevant.

\section{Julis Giofredi, CBp.}

Syn.: Lubrus Julis, Brunn.; Labrus Giofredi, Riss.; Julis Giofredi, Riss., Cuv. et Valenc., Guichen., Canestr.; Coris Giofredi, Guinth.

Nom vulgar: Donzella, Tarraguna, Menorca; Senyoreta, Donzezella, Barcelona; Senyoreta, Güiula, Costa de Lle vant. 


$$
\text { Var. Julis festiva, Valenc. }
$$

Syn.: Julis festiva, Cuv, et Valenc., Guichen.

Nom vulgar: Donzella, Tarragona, Menorca; Senyoreta, Güiula, Costa de Llevant,

\section{Julis pavo, Günth.}

Syn.: Labrus pavo, Lacep., Hasselquist; Labrus hebraicus, Riss.; Julis turcica, Riss., Lowe, Canestr.; Julis pavo, Cuv. et Valenc., Guichen.; Chloricthys pavo, CBp.

Nom vulgar: Donzella, Tarragona; Senyoreta, Güiula, Costa de Llevant; Vit d'en Gahona, Menorca.

Género, XyRichthys, Cuv.

Xyrichthys novacula, Linn.

Syn.: Novacula cultrata, Günth.; Rason, Cuv.; Xyrichthys cultratus, Cuv. et Valenc., Guichen.; Novacula coryphoena, Riss.; Coryphona novacula, Lacep., Bonnat., Linn., Rosenthal; Novacula pis. cis, Gesner, Willugh.; Novacula, Rond.; Xyrichthys novacula, CBp., Canestr.

Nom vulgar: Rahó, Barcelona, Tarragona, Costa de Llevant, Menorca.

Familia dels Pomacentrida.

Género, Chromis, Cuv.

Chromis Castanea, Cuv.

Syn.: Heliases Chromis, CBp.; Heliastes Chromis, Günth.; Chromis Castanea, Nordmann, H. Schinz, Riss., Guichen.; Heliases limbatus, Cuv. et Valenc.; Sparus C.hromis, Lacep., Riss., Bonnat., Linn.; Chromis, Rond., Gesner, Willugh.; Castaneus piscis, Bell.

Nom vulgar: Castanyoleta, Tarragona; Cigala, Barcelona; Muret, Menorca.

El Pixano (Pagellus breviceps. CBp.) y aquest peixet son, com tẻnim dit, els que mes alegren les manses aygues de nostres cales y ports. 
Tribu dels Acunthopterrgienchs Abdominals.

Familia dels Gasterosteidoe, CBp.

Género, Gasterosteus, Linn.

Sub-género, Gasterosteus.

Gusterosteus teiuras, Cuv, et Valenc.

(Var. del Gasterosteus aculeatus, „Linn.)

Syn.: Gusterosteus eleguns, Blanch.. Sauv.; Gasterosteus argentatissimus, Blanch., Sauv.; Gasterosteus Bailloni, Blanch., Sauv.; Gasterosteus leiurus, Blanch., Soland, CBp., Gehin, Sauvage.

Nom vulgar: Surell, Banyoles. Varietat nova per Catalunya; habita en la Cequia Comtal y aygua molls de Cân Tunis (Barcelona), tolls y rechs del Baix Vallés, sobre tot, en els gorchs de poca fondaria y rechs llotosos y plens d'herba, escassejant en les corrents; rius Besós y Tenes, entre Mollet y Montmeló, etz.

Familia dels Aulostomida.

Género, Centriscus, Linn, Centriscus scolapax, Linn.

Syn.: Solenostomus scolapax, Riss.; Centriscus scolapax, Lacep., Riss., Brunn., Bloch, Cuv., Rosenthal, CBp., Günth., Canestr.: Scolapax, Aldrov., Willuhgh.; Becada, Rond., Bonnat.

Nom vulgar: Trompeté, Tarragona, Barcelona, Menorca; Musich, Torredembarra, Menorca.

Familia dels Mugilida.

Género, MugIL, Arted.

Mugil cephalus, Cuv, et Valenc.

Syn.: Mugil provensalis, Riss ; Cabot, Rond.; Mugil cephalus, Riss., Del., Agass., CBp., Günth., Canestr., Blanch., Guichen.

Nom vulgar: Llissa llubarrera, Tarragona; Llissara llubarrera, Barcelona; Llissa, Taberner, Menorca.

Mugil auratus, Riss.

Syn.: Mugil aurafus, CB̉p., Lowe, Günth., Canestr., Cuv. et Valenc., Guichen.

Nom vulgar: Galta roig Tarragona, Menorca; Llissara, Barcelona. 
Anys enrera, a l'encanyiçada en una sola llevada a vegades se'n pescaven d'aquestes llisses més de 2,000 kilograms.

$$
\text { Mugil capito, Cuv. et Valenc. }
$$

Syn.: Mugil octoradıatus. Günth.; Mugil ramada; Riss.; Rama do, Riss.; Mugil çapito, CBp., Günth., Canestr., Guichen., Blanch.

Nom vnlgar: Capsut, Llissa de roquer, Tarragona; Llissara, Barcelona; Llissa agut, Llissa cap pla, Menorca.

\section{Mugil saliens, CBp.}

Syn.: Mugil saliens, Riss., Cuv. et Valenc., Guichen., Gûnth., Canestr.

Nom vulgar: Sama, Tarragona. Especie nova per Catalunya.

$$
\text { Mugil labeo, Cuv. et Valenc. }
$$

Syn.: Mugil provengalis, Riss.; Mugil Sabounie, var., Riss.; Mugil labeo, CBp., Günth., Canestr.

Nom vulgar: Caluga, Tarragona; Llissara, Barcelona; Llíssa-Galubet, Menorca.

\section{Mugil Chelo, Cuv.}

Syn.: Mugil septentrionalis, Günth.; Mugil labrosus, Riss.; Mugil cephalus, Riss.; Mugil cephalus, var. B, Delaroche; ChaInc, Rond.; Mugil Chelo, Cuv. et Valenc., Guichen., CBp., Günth., Schlegel, Canestr.

Nom vulgar: Llissa vera, Tarragona, Menorca; Llíssara, Barcelona.

A la Llissa petita o de cria se li dona a Tarragona'l nom de Llissó y a Barcelona'l de Tomba navios.

\section{Familia dels Atherinidie. Género, ATHERINA. Atherina hepsetus, Linn.}

Syn.: Atherina hepsetus, Cuv. et Valenc., Guichen., Lacep., Riss., Bloch, Del., CBp., Günth., Canestr.; Sauclés, Rond.

Nom vulgar: Peix sense sang, Tarragona; Xanguet, Xasclet, Barcelona; Joell, Costa de Llevant; Serclet, Menorca. 
Atherina Boveri, Riss.

Syn.: Atherina Bo!eri, CBp., Günth., Canestr., Cuv. et Valenc., Guichen.: Atherina hepsetus, var. 3 Delaroche; Joel, Rond.

Nom vulgar: Asa, Aseta, Tarragona; Xanguet, Barcelona; Cabessuda, Menorca.

Atherina Mochon, Cuv, et Valenc.

Syn.: Atherina Mochon, Günth., CBp., Canestr., Guichen.; Atherina hepsetus, var. 2 Delaroche.

Nom vulgar: Peix sense sang, Tarragona; Joell, Costa de Llevant; Muxó, Menorca.

\section{Familia dels Sphyraenida. \\ Género, Sphyraena, Kleín. \\ Sphyraena Spet, Lacep.}

Syn.: Sphyraena vulgaris, Günth., Canestr., Cuv. et Valenc., Guichen.; Sphyraena Spet, CBp., Riss.; Esox sphvraena, Linn.' Bloch, Brunn.: Spet, Rond.; Sphyraena, Bell., Salvian.

Nom vulgar: Espet, Tarragona, Menorca.

Sub-ordre dels Malacopterygienchs.

Tribu dels Malacopterygienchs Pseudapodes.

Familia dels Ammodytıdae.

Género. А м моDY теS, Arted.

Ammodytes cicerellus, Rafin.

Syn.: Ammodịtes tobianus, Costa, Can€str., Riss.; Ammoditis Siculus, swainson, CBp., Günth.; Ammodytes argenteus, Riss.; Cicerellus messaniensis, P. Boccone.

Nom vulgar: Sonsu, Trencavits, Barcelona; Enfú, Menorca. Es. pecie rara.

L'A. tohianus, Linn, que cita en Sánchez Comendador, proba. blement será l'A. cicerellus, Rafin.

\section{Familia dels Ophidiidae}

Género, O phidium. Arted.

Ophidium barbatum, Linn.

Syn.: Ophidium Barbalum, Cuv., Guichen., Lacep., Riss., Bonnat., Brunn., Broussonet, Bloch, Rosenthal, Agass., J. Muiller, CBp., 
Kaup, Günth., Costa, Jobert, Canestr.; Grillus vulgaris, Bell.

Nom vulgar: Pixota blanca, Barcelona, Tarragona; Metge, Costa de Llevant; Pamfont, Congre dolç, Menorca.

\section{Ophidium Vassallii, Riss.}

Syn.: Ophidium barbatum, Costa; Ophidium Broussonetii, J. Müller; Ophidium Vassallii, J. Müller, CBp., Kaup, Günth., Canestr., Cuv., Guichen.

Nom vulgar: Pixota, Tarragona; Metge, Costa de Llevant; Pamfont vermey, Menorca.

Género, Fierasfer, Cuv.

Fierasfer imberbis, CBp.

Syn.: Fierasfer acus, Kaup, Günth., Canestr.; Fierasfer Fontanesii, Costa; Fierasfer imberbis, J. Müller; Ophidium imberbe, Cuv., Linn.; Ophidium Fierasfer, Riss.; Notopterus Fontanesii, Riss.; Gymnolus acus, Brunn.; Ophidium flavum vel ophidium imberbe, Rond.

Nom vulgar: Pixota de llanguet ó de carall de Jan, Tarragona; Fi de Murena mansa, Menorca.

Aquesta especie viu parássita dintre l'holoturit pedata de la familia dels Aspidochiroto, Stichopus regalis, Cuv. (Llanguet). No l'havem trobat dintre 1'Holothuria tubulosa, GML. (Carall de Jan) de la mateixa familia, ni dintre la Cucumaria Cucumis, Riss. (Cohombre mari) de la familia dels Dendrochiroto.

Tribu dels Malacopterygienchs Sub-branquials.

Familia dils Gadidoe. Sub-familia dels Gadini.

Género, Gadus.

Gadus minutus, Linn.

Syn.: Morua capelanus, Riss.: Gadus luscus var., Delaroche; Asellus mollis minor, Willugh,; Gadus minutus, Brunn., Bloch, Costa, Fries et Ekström, Nilsson, CBp., Günth., Canestr.

Nom vulgar: Móllera, Barcelona, Tarragona; Capellá, Costa de Llevant, Menorca. Abunda molt. 


\section{Género, Merlangus, Cuv. \\ Merlangus Poutassou, Riss.}

Syn.: Merlangus vernalis, Canestr.; Merlangus melanostomus, Valenc.; Merlangus communis, Costa, Canestr.; Merlangus voutassou, CBp.. Günth.; Gudus merlangus, Riss.

Nom vulgar: Llússara, Tarragona; Maira, Torredembarra: Peix Rey, Menorca. Peix de fonera.

\section{Merlangus al genteus, Vaill.}

Syn.: Gadiculus argenteus, Guichen., Günth., Giglioli; Gadıs argenteus, Belloti, Günth.

Nom vulgar: Ulls, Tarragona. Peix de fonera. Especie nova per Catalunya.

Sub-familia dels Morini.

Género, MoRA, Riss.

Mora Mediterranea, CB p.

Syn.: Gadus moro, Riss.; Mora mediterranea, Riss., Günth., Canestr., Brit. Capel.

Nom vulgar: Mollera moranella, Tarragona. Especie molt rara, nova per Catalunya.

\section{Sub-familia dels Merlucini. \\ Género, Merlucius, Cuv. \\ Merlucius vulgaris, Costa.}

Syn.: Merlucius esculentus, CBp., Canestr., Riss.; Merlucius vulgaris, CBp., Günth., Cuv.; Gadus merlucius, Linn.; Brunn., Bloch, Schlegel, Lacep., Riss.; Asellus, Salvian., Willugh., Aldrov.

Nom vulgar: Llús, Barcelona, Tarragona, Menorca. Abunda molt. Havem vist llussos pescats á palangre de $10 \mathrm{y}$ mes kilograms de pes.

Com especie o varietat nova, en 1909 ens presentaren un exemplar que tenia un color de palla brillant, nacari, amb les aletes rosses com un fil d'or (Llus ros), y no resultá pas aixi, car el peix patía 
una aglobulia ó forta anemia, amb alteració pigmentaria per insuficiencia de l'hematosis, puix li trobarem á dreta y esquerra agarrats. fortament á les branquies fins á set cuquets purpurins (Femella del crustaci siphonostomit Lernoa branchialis, Linn., de 0’010 á 0.012 $\mathrm{m}$. de llargaria que á faisó de sangoneres li xuclaven la sang, cuquets que també havem trobat examinant altres llussos en apariencia sans, pero amb nuclis indurits y decoloració de l'extrem lliure de les branquies á causa d'inflamacions intersticials localisades y cróniques.

Género, Uraleptus, Costa.

Uraleptus maraldi, Costa.

.Syn.: Gadıs maraldi, Riss.; Merlucius maraldi, Riss.; Uraleptus maraldi. CBp., Günth., Canestr.

Nom vulgar: No'n te.

L'Institució Catalana d'Historia Natural ne serva un exemplar pescat a Tarragona'l 9 de Novembre de 1910 y nosaltres ne servem qualques altres pescats á l'artet el 8 de Juliol de 1911.

Sub-familia dels Lotini.

Género, Lota, Cuv.

Lot a molva, CBp.

Syn.: Molva vulgaris, Günth.; Gadus molva, Lacep.,Linn., Bloch, Schlegel; Asellus longus, Willugh.

Nom vulgar: Llengua de bacallá. Peix de fonera. Especie nova en el Mediterrá. Era pel mes de Janer de 1910 que a la Pescateria de Tarragona 'ns crida l'atenció un peix de 14 kilograms que no poguerem adquirir, car el venien a terces o kilos. Aquest peix, per la seva forma y dimensions no podia ser mes que aquesta especie, y'l 31 de Maig del propi any sortosament ne poguerem adquirir un exemplar de 15 kilograms, agafat com l'altre a palangre, que vingué a confirmar plenament les nostres sospites. Haviem sentit parlar als vells palangrers de la llengua de bacallá, mes no s'ens havía presentat ocasió fins allavors de poguerla veurer.

Lota elongata, Riss.

Syn.: Molva elongata, Günth., Canestr.; Lota molva, Costa; Lota elongata, CBp., Canestr.; Gadus molva, Riss. 
Nom vulgar: Escolá, Barcelona, Tarragona. Peix de fonera.

Género, Phycis, Arted.

Phycis blennoides, Günth.

Syn.: Phycis blennoides, Canestr., Riss., Bl. Schneid., CBp.; Phicis blennioide, Guichen.: Phy'cis furcatus, CBp.; Phycis Gme. lini, Riss.; Batrachoides Gmelini, Riss.; Blennius gadoides, Lacep.. Riss.; Phycis tinc'a, Bl. Scheid.; Gadus blennoides, Brunn.

Nom vulgar: Mollera Brótola, Tarragona; Móllera pigada, Barcelona; Mollera, Menorca. Peix de fonera.

Phycis mediterraneus, Delaroche.

Syn.: Phycis tinca, CBp.; Phycis limbatus, Valenc.; Blennius phịcis, Riss.; Phycis mediterrancus, Riss., Guichen., Costa, Lowe, Giinth., Canestr.; Tenca marina, Cuv., Salvian., Willugh.

Nom villgar: Móllera roquera, Barcelona, Tarragona: Mollera, Menorca.

$$
\text { Género, Motella, Cir. }
$$

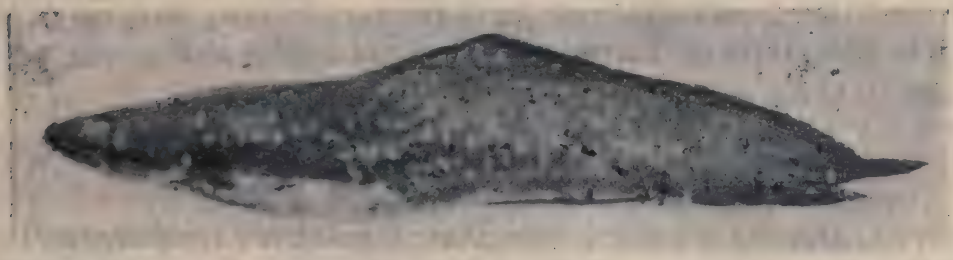

Motella tricirrata, Günth.

Syn.: Onos mustclla, Riss.; Gudus mustella, Riss.; (iadus tricirratus, Bloch; Mustella marina, Willugh.

Nom vulgar: Fura, Tarragona. Especie nova á Catalunya y no en el Mediterrá com tenin dit.

El primer exemplar el vegerem el 21 de Juny de 1910 y d'allavors ençà n'havem examinat qualques altres, tots pescats a l'artet, la major part en aygues de la Platja llarga de Tarragona.

Molella maculata, Costa.

Syn.: M-tella communis, Canestr.; Motella maculata, Gïnth., Ris.s.: Hotella medilerranea, CBp.; Onos maculata, Riss. 
Noin vulgar: Fura blanca, Tarragona; Palayó, Barcelona; Guineu, Costa de Llevant: Mollera borda, Menorca.

Motella fusca, CBp.

Syn.: Motella communis, Costa; Onos fusca, Riss.; Motella fusca, Riss.

Nom vulgar: Fura negra, Tarr .gona; Palayó, Barcelona; Guineu Costa de Llevant; Mollera inglesa, Menorca.

\section{Familia dels Macrourida. \\ Género, MACROURUS, Bloch. \\ Macrourus Coiorynchus, CBp.}

Syn.: Lepidolep us colorhynchus, Guichen., Cuv., Riss; Macrourus colorhynchus, Costa, Günth., Canestr.

Nom vulgar: Titulot. Peix de fonera. Especie nova per Catalunya.

Els exemplars que havem examinats en tres anys, tots estavem bruts del llot de l'arrastre. El 28 de Janer de 1913 nos poguerem convencer que aquesta especie no es tan rara com es aixó, car hi havia a la Pescateria de Tarragona tot un vol agafat al bou que pesaria uns 10 kilograms.

\section{Macrourus Trachỵrhỵchus, Günth.}

Syn.: Macrourus trachyrhynchus, Canestr.; Lepidoleprus trachurhlynchus, Guichen., Cuv., CBp., Canestr., Riss.; Oxpcephas scabrus, Rafin"; Mysticetus, Aldrov.

Nom vulgar: Titulot, Tarragona. Peix de fonera. Especie nova per Catálunya.

L'Institució Catalana d'Historia Natural ne serva un exemplar momificat, ad fuirit per nosaltres a Tarragona'l 8 d'Abril de 1910.

\section{Fanilila dels Pletironectido. \\ Género, Limanda, Gottsche. \\ Limanda vulgaris, Gottsche.}

Syn.: Limanda oceanica, CBp.; Platessa limanda, Cuv.; Pleuronectes limanda, Lacep., Linn., Bloch, Günth., Schlegel;Limanda, Bell., Rond., Duham., Bonnat. 
Nom vulgar: no'n té. Sabém no mes, per referencies, que se la senyala en les nostres aygues, peró en Moreau no la cita en el Mediterrá.

\section{Género, Flesus.}

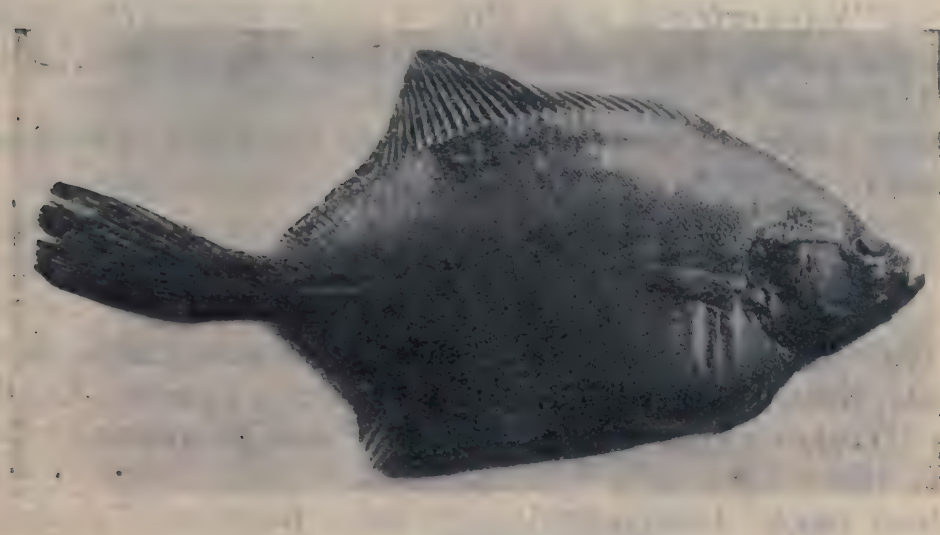

Flesus vulgaris, Moreau.

Syn.: Pleuronectes flesus, Blanch., Lacep., Bonnat., Linn., Bloch, Nilsson, Günth., Schlegel; Platessa flesus, Gottsche, CBp. Siebold; Pleuronectes passer, Bloch; Passer fluviatilis, Bell.

Nom vulgar: Rèmol de riu. Especie nova en el Mediterrá. En Moreau no la senyala en les nostres aygues marines.

Els pochs exemplars examinats se distingeixen de l'especie seguent, car la línea lateral, quasi en tnt el seu trajecte, està vorejada dascates molt aspres, la cua te menys alçaria que llargaria y l'anal amb 41 ratgs. essent ai xis que la del $F$. passer, $M$., ne te quant menys 43.

\section{Flesus Passer, Moreau.}

Syn.: Pleuronectes italcus, Günth; Platessa passer, CBp., Costa. Canestr.; Pleuronectes pusser, Riss.; Pleuronectes flesus var., Delaroche; Passer, Rond.

Nom vulgar: Rèmol de riu. Tarragona, Desembocadura del Francoli, etz. 


\section{Género, Solea, Cuv. \\ Solea vulgaris, Gottsche.}

Syn.: Solea vulgaris, Riss., CBp., Canestr., Günth.; Pleuronectes solea, Lacep., Riss., Bonnat.. Linn.. Brunn., Bloch; Solea, Bell., Rond., Duham.

Num vulgar: Llenguado, Pelayíl petit, Barcelona, Tarragona, Menorca.

\section{Solea Lascaris, CBp.}

Syn.: Solea impar, Günth.; Solea aurantiaca, Günth.; Solea nasuta, Nordm., CBp., Kaup; Solea Lascaris, Canestr., Günth., Riss., Guichen.; Pleuronectes nasutus, Pallas; Pleuronectes Lascaris, Riss.

Nom vulgar: Llenguado. Pelayi'l petit, Barcelona, Tarragona, Menorca.

\section{Solea Kleinii, CBp.}

Syn.: Rhombus Kleinii. Riss.؛ Solea Kleinii, Costa, Kaup, Canestr., Günth.

Nom vulgar: Llenguado, Pelayill petit, Barcelona, Tarragona, Menorca.

\section{Solea oculata, Willugh.}

Syn.: Solea ocellata, Günth., Canestr.: Solea oculata, Riss., Valenc., Guichen., CBp., Canestr., Rond., Duham.; Plenronectes ocellatus, Riss.; Pleuronectes pegusa, Lacep.

Nom vulgar: Llenguado, Pelayíl petit, Tarragona: Llengua de Sant Pau, Menorca.

Havem observat una var. que'n lloch de presentar set taques negres, arrodonides y enrevoltades de puntets grochs, no mes ne presenta cinch, coneixéntsela vulgarment a Mahó amb el nom de Perdiu de Sant Pere. 
Sub-género, Microchirus. CBp. Microchirus luteus, CBp.

Syn.: Solea lutea, CBp., Canestr., Günth.; Pleuronectes $l u_{\text {r }}^{\text {त }}$ tells, Riss.; Rhombus luteus, Riss.

Nom vulgar: Llenguado, Pelayíl' petit. Barcelona, Tarragona.

Microchirus variegatus, Moreau.

Syn.: Solea variegata, Günth.; Solea mangili, CBp., Canestr.; Monochirus microchirus, Guichen.; Microchirus lingula, CBp.: Rhombus mangili, Riss.; Pl. uronectes mangili, Riss.; Pleuronectes microchirus, Delaroche.

Nom vulgar: Llenguado d'arena. Tarrägona; Peluda, Menorca.

Sub-género, Monochirus, Rafin.

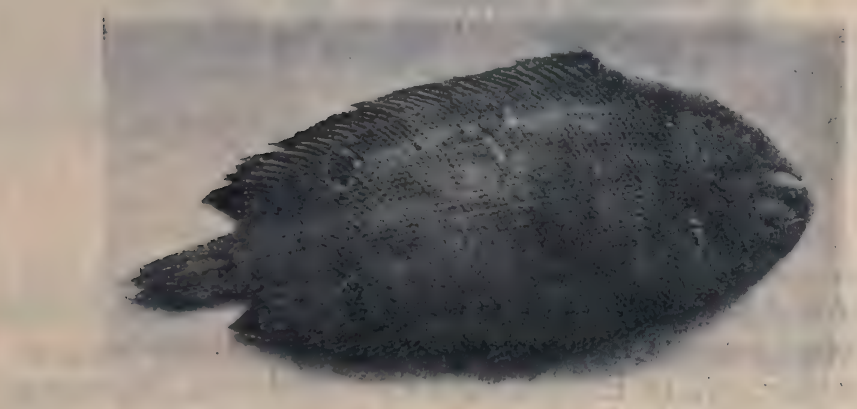

Monochirus hispidus, Rafin,

Syn.: Monochirus hispidus, CB|)., Costa; Solea Monochur, CBp., Gïnth., Canestr.; Monochirus pegusa, Riss., Guichen.: Pleuronectes pegusa, Riss.

Nom vulgar: Llenguado de fonera, Barcelona; Sanremus, Menorca. Ne servém un curiós exemplar pescat el 18 de Febrer ća 1911 que ofereix en la meytat posterior del costat dret quatre taquetes rodones, simetriques y negres enrevoltades de puntets blanquissims. Sobre'l dors o cara dreta de qualques Llenguados hi havem trobat el petit molusch Modiola discrepans, Lm. K. 


\section{Género. Pleuronectes.}

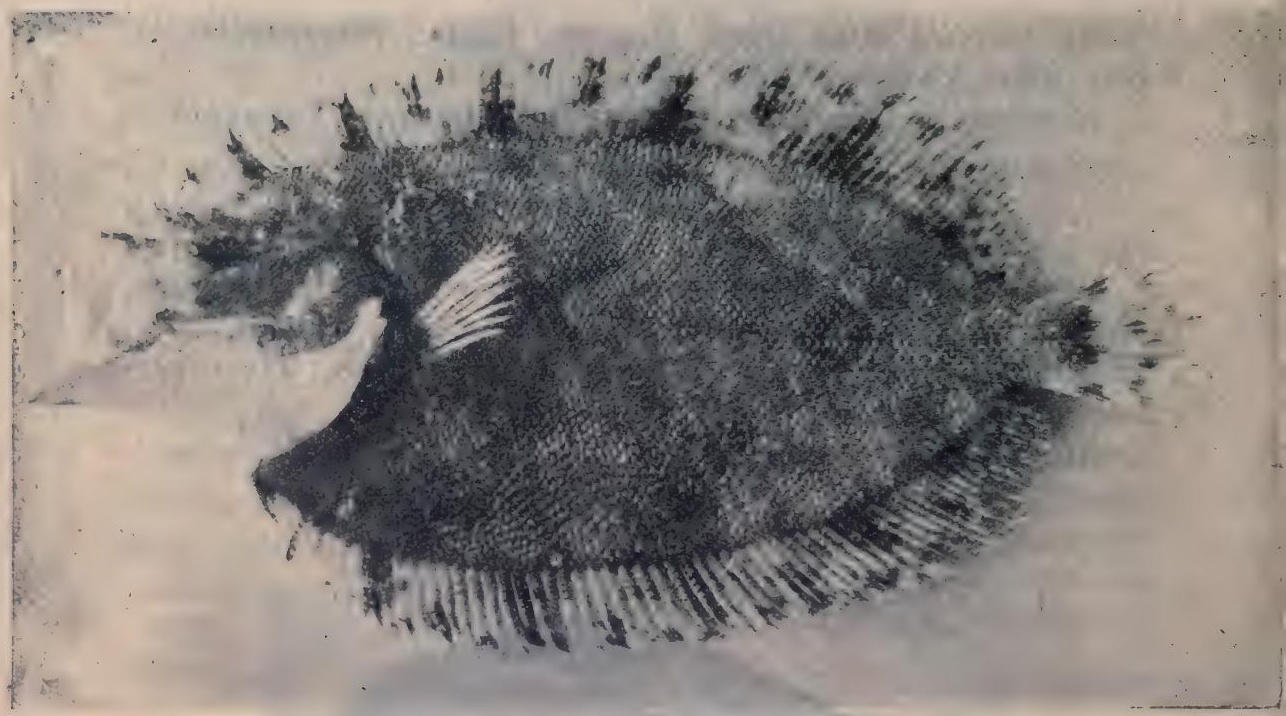

Pleuronectes Hirtus, Abilgaard.

Syn.: Rhombus punctatus, Günth.; Rhombus hirtns, Nilsson.; Scophthalmus hirlus. C.Bp.; Zengopterus hirtus, Gottsche,

Nom vulgar: no'n té. Especie nova en el Mediterrá. Moreau no la senyala en nostre mar, pero nosaltres n'havem adquirit tres exemplars, dos d'ells pescats a l'artet a sol-ixent de Tarragona, prop la Torra de la Mora (Platja llarga) 'l 20 de Juny de 1910 y l'altre segurament del Bou, recullit a la Pescatería'l 12 del prop-vinent Novembre y que serva l'Institució Catalana d'Historia Natural.

Pleuronectes unimaculatus, Moreau.

Syn.: Pleuronectes punctatus, Bloch, Bonnat.; Phrynorhombus unimaculatus, Günth., Canestr.; Scophthalmus punctatus, CBp., Riss., Nilsson.

Nom vulgar: Pelaya miseries, Tarragona; Serrandell, Goles de l'Ebre. 


\section{Pleuronectes Grohmanni, CBp.}

Syn.: Arnoglossus Grohmunni, Günth., Canestr.; Pleuronectes Grohmanni, Canestr.

Nom vulgar: Pelaya rossa, Tarragona; Serrandell, Goles de I'Ebre; Llenguado, Menorca.

\section{Pleuronectes arnoglossus, CBp.}

Syn.: Arnoglossus laterna, Günth., Canestr.; Pleuronectes arnoglossus, Canestr.; Rhombus nudus, Riss.; Pleuronectes Leotardi. Riss.; Pleuronectes casurus, Pennant, CBp.; Pieuronectes laterna, Arted.; Arnoglossus, Gesn., Willugh.

Nom vulgar: Pelaya rossa, Barcelona, Tarragona; Serrandell, Gcles de l'Ebre.

\section{Pleuronectes conspersus, Canestr.}

Syn.: Pleuronectes conspersus, Günth.. Brit. Capel.

Nom vulgar: Pelaya-miseries, Tarragona; Serrandell, Goles de l'Ebre. Especie nova per Catalunya.

\section{Pleuronectes Boseii, CBp.}

Syn.: Arnoglossus Boscii, Günth., Brit. Capel., Canestr.;Plellronectes Boscii, Riss., Canestr., Cuv.; Hipoglossus Boscii, Riss.

Nom vulgar: Pelaya-bruixa, Tarragona, Costa de Llevant; Serrandell, Goles de l'Ebre. Especie nova per Catalunya.

\section{Pleuronectes megastoma, Donov.}

Syn.: Rhomhus megastoma, Nilsson, Günth.; Plearonectes megastomus, CBp.; Pleuronectes megastoma, Schlegel; Pleuronectes cardina, Cuv.: Grande Calimande, Duham.

Nom vulgar: Pelaya bruixa, Tarragona, Costa de Llevant; Serrandell, Goles de l'Ebre; Capellá, Menorca.

\section{Pleuronectes Citharus, CBp.}

Syn.: Citharus linguatula, Giïnth., Canestr.; Hipoglossus citharus, Costa, Riss.: Hipoglossus macrolepidotus, Cuv.; Pleuro- 
nectes citharus, Max. Spinola: Pleuronectes macrolepidotus, Del., CBp., Canestr.; Citharus, Rond., Gesner, Willugh.

Nom vulgar: Pelaya-guarda la vida, Tarragona; Pelaya, BarceIona; Capellá, Rénıl, Menorca.

Género, Rномвus, Klein.

Rhombus maximus, Riss.

Syn.: Psetta maxima. CBp.: Rhombus maximus, CBp., Costa, Canestr., Gunth.: Pleuronectes maximus, Lacep., Riss., Linn., Brunr., Bloch, Schlegel; Rhomhus aculeatus, Duham., Rond., Gesner. Willugh., Gottsche; Turbot, Bonnat., Cuv., Guichen.: Rhombus, Bell.

Nom vulgar: Rémol empetxinat, Tarragona. Especie comuníssima y que abunda en els mercats, y no citada en cap catálech de peixos que's refereixi a Catalunya.

\section{Rhombus lovis, Rond.}

Syn.: P'setta rhombus, CBp.; Rhombus vulgaris, Costa; Rhombus barbatus, Riss.; Pleuronectes rhombus, Lacep., Riss., Bonnat., Linn., Brunn., Bloch, Schlegel; Rhombus Lovis, Gesner, Willugh., Gottsche, CBp., Canestr., Günth.; Rhombus alter Gallicus, Bell.

Nom vulgar: Rémol, Barcelona, Tarragona; Rom, Costa de Llevant.

Existeix una var. que presenta la particularitat de tindrer menys dividits els primers ratgs de la dorsal.

Género, BHotus, CBp.

Bothus rhomboides, CBp.

Syn.: Rhomboidichthys mancus, Günth., Canestr.; Rhombus rhomboides, CBp., Canestr.; Rhornbus mancus, Riss., Guichen; Pleuronectes mancus, Riss.; Rhomboides, Rond.

Nom vulgar: Puput, Tarragona; Rémol, Barcelona; Pedás, Menorca. Notarém que un exemplar d'aquesta especie adquirit el 15 de Maig de 1910 tenia iguals les dugues cares, aixó es, com la del costat esquerra o dels ulls.

Bothus podas, CBp.

Syn.: Rhomboidichthys podas, Günth., Canestr.; Rhombus po- 
dus, CBp., Costa, Guichèn., Canestr.; Rhombus Gesneri, Riss.; Pleuronectes argus, Riss.; Pleuronectes podas, Del.

Nom vulgar: Puput. Tarragona; Rèmol, Barcelona; Pedas, Menorca.

$$
\begin{aligned}
& \text { Género, Plagusia. } \\
& \text { Plagusia lactea, CBp. }
\end{aligned}
$$

Syn.: Plagusia lutea, Canestı., Costa: Ammopleurops lacteus, Günth.

Nom vulgar: Rèmol blanch, Tarragona. Especie nova per Cata. lunya.

Familia dels Cyclopterida.

Sub-familia del Cyclopterini.

Género, LePAdogaster, Goüan.

Lepadogaster Goüanii, Lacep.

Syn.: Lepadogaster biciliatus, Riss., Nordm.; Lepadogaster Balbis, Rosenthal, Costa, CBp., Riss.; Lepadogaster Goüanii, Riss.. Cuv., Guichen., Costa, CBp.; Brisout de Barneville, Günth., Canestr.; Lepadogaster rostratus, Bl. Schneid., CBp.; Cyclopterus lepadogaster, Bonnat.

Nom vulgar: Xucladó, pega roques, Tarragona; Peix porch, Barcelona: Xucla dit, Cabot xuclador, Menorca. Especie rara.

\section{Lepadogaster Candolii, Riss}

Syn.: Mirbelia Decandollii, Canestr.; Lepadagoster Rafinesquii, Costa: Lepadogaster olivaceus, Riss.: Lepadogaster Jussieni, Riss.; Lepadogaster Candolii, Cuv., Guichen., Brisout, Günth.

Nom vulgar: Xucladó, Pega-roques, Tarragona; peix porch, Barcelona, Menorca. Especie rara.

\section{Lepadogaster bimaculaus, Flem.}

Syn.: Lepadoguster reticulatus, Riss.: Mirbelia Desfontainii, Canestr.: Lepadngaster Mirhelii. Riss.; Lepadogaster Desfontainii, Riss.; Lepadngaster ocellatus, Riss., Guichen.; Lepadogaster bimaculatus, CBp., Brisout, Nilsson, Günth.; Cyclopterus bimaculatus, Lacep.. Bol „nat., Pennant. 


\section{Fauna ictiológica de Catalunya}

Nom. vulgar: Xucladó, Pega-roques, Tarragona; Peix porch, Barcelona.

Tribu dels Malacopterygienchs abduminals.

Familia dels Cyprinido.

Sub-familia dels Cyprinin, CBp.

Género, CyPrinus.

Subgénero, CARPA (CYPRINUS)

Cyprinus Carpio Linn.

Syn.: Cyprinus Carpio, Cuv. et Valenc.. Blanch., Lacep., Bloch, Costa, CBp., Nilsson, Heckel et Kner, Siebold, Canestr., Gunth,, Schlegel.

Non vulgar: Carpa d'Holanda.

\section{Var. Cyprinus specularis, Lacep.}

Syn.: Cyprinus rex c!prinorum, Blanch., Bonnat.; Cyprinus macrolepidotia, Hartmann.

Nom vulgar: Carpa de mirall.

\section{Var. Cyprinus hungaricus, Heckel.}

Nom vulgar: Carpa de Bohemia.

\section{Var. Cyprinus Hí Goi.}

Nom vulgar: Carpa del Japó.

\section{Var. Clprinus Kollarii, Heckel et Kner.}

Syn.: Cyprinus Kollarii, CBp., Cuv. et Valenc., Blanch., Gehin; Cyprinus striatus, Holandre, Selys-Longchamps, CBp.

Nom vulgar: Carpa de Franconia, Carpa de Turinga.

Amb aquesta especie y varietats, totes de l'Aquarium y laboratori ictiogènich del Park de la Ciudadela de Barcelona, s'ha emprès baix la entesa direcció d'en Francesch d'A. Darder la repoblació piscícola en diferents it:drets de Catalunya: Estany de Banyoles, de Tarrasa (Guitart), de Lleyda; rius, Llobregat, Cardoner y altres de Lleyda, etz. 
Sub-género CaRAssius, Nilsson.

Carassius vulgaris, Nilsson.

Syn.: Cyprinus carassius, Cuv. et Valenc., Bonnat., Lacep., Linn., Bloch, Hermann, Fries, Ekström et Sundevall, Schlegel: Carassius Lincei, CBp.; Carassius vulgaris, Nordmann, Heckel et Kner, Siebold, Canestr., Günth.; Carassius, Willugh.

Nom vulgar: Carpa carassina. Aquarium y Laboratori ictioge- . nich del Park de Barcelona, y espargida en els mateixos indrets.

Carassius auratus, Günth.

Syn.: Carassius auratus, Canestr.; Cyprinopsis auratus, Blanch., Gehin, Soland; Cyprinus auratus. Cuv. et Valenc., Vallot, Lacep., Riss., Bonnat., Linn., Bloch.

Nom vulgar: Peix de bassa (King-yu.) Peixeres, basses, surti. dors, etz.

Dintre'l mar, pescat a l'artet pel Maig de 1911, adquirirem un raro exemplar que servem, no sabem si de aygua dolça o del mar. car poch dies avans havia plogut y baixat els rius y'ls barranchs. Els pescayres el confonen donantli'l nom de Tenca.

Es de un hermos color d'or vell y ofereix la particularitat de tindrer les anals pariones cum les ventrals y pectorals. Mideix $0^{\prime} 18 \mathrm{~m}$. de llargaria $\times 0^{`} 6 \mathrm{~m}$. de passo d'alçaria; '1 diámetre de l'ull $0^{`} 008 \mathrm{~m}$.; l'espay interorbitari $0^{\prime} 018 \mathrm{~m}$. y'l cap $0^{\prime} 04 \mathrm{~m}$.; la dorsal te 3/19, pero'l primer y segon raigs forts rudimentaris lo mateix que'l primer y segón de les anals.

Género, Barbus, Cuv. Barbus fluviatilis, Agass.

Syn.: C'yprinus barbus, Linn., Bloch, Schlegel; Barbus fluviatilis, Cuv. et Valenc., Blanch., CBp., Heckel et Kner, Siebold, Canestr., Gunth.; Barbus, Rond., Duham., Bonnat., Lacep., Bükner, Vallot.

Nom vulgar: Barb. General en els nostres rius y estanys.

Barbus meridionalis, Riss.

Syn.: Burbus caninus, CBp., Heckel et Kner, Canestr., Günth., 
Cuv. et Valenc.; Barbus meridionalis, Blanch.; Cyprinus barbus, Riss.

Nom vulgar: Barb. Mes limitada que l'especie anterior.

\section{Género, Tinca, Cuv.}

Tinca vulgaris, Cuv.

Syn.: Tinca itálica, CBp.; Tinca vulgaris, Costa, CBp., Heckel et Kner, Siebold, Canestr., Günth., Cuv. et Valenc., Blanch.; Tinca Chrysitis, Agass., Selys-Longchamps, CBp.; Cyprinus tincauratus, Lacep.; Cyprinus tinca, Lacep., Linn., Bloch, Schlegel; Cyprinus tinca-auratus, Bloch, Bonnat., Vallot; Tinca aurata, Schinz; Tinca, Bell., Salvian., Duham., Bonnat., Jurine, Vallot.

Nom vulgar: Tenca. Riu Ebre, estanys de Banyolas, d'Ibars y altres de Lleyda, etz.

Se creu fundadament que les tenques dels estanys de Lleyda hi foren tirades pels frares avans de l'any 1835.

La Tenca (viridescens) verda o del Cáucas y la (rurata) roija ó de Mongolia amb la 1 ostra, provinentes totes de l'Aquarium y La. boratori ictiogénich del Park de Barcelona, pertanyen al nombre d'especies repobladores.

S'han llençat també en qualques indrets les especies: Globio flu viatilis, Agass.; Abramis Brama, Agass.; Alburnus lucidus, He ckel et Kner; Scardinius erythrosthalmus, Heckel et Kner; Idus melanotus, Heckel et Kner y Cobitis fossilis, Linn.

\section{Sub-familia dels Leuciscini. \\ Género, SQUALIUS. \\ Squalius Cephalus, Blanch.}

Syn.: Leuciscus cephalus, Günth.;Squaiius clathratus,Blanch.; Squalius meridionalis, Blanch.; Squalius cephalus, Blanch., Soland, Lunel, Siebold; Leuciscus dobula, Bert, Cuv. et Valenc., Marcotte; Agass., Selys-Longchamps; Leuciscus latifrons, Nilsson; Squalius dobula, Heckel et Kner; Squalius cavedanus, CBp., Heckel et Kner, Canestr.; Leuciscus cavedanus, Cuv. et Valenc., CBp., Filippi; Leuciscus cavede, Riss.; Gardonus cephalus, CBp.; Cyprinus dobula, Vallot; Cyprinus Jeses, Jurine; Cyprinus chup, Bonnat.; Cyprinus idus, Bloch; Cyprinus cephalus, Linn., Fries et EKström; Cephalus fluviatilis, Rond. 
Nom vulgar: Bagre, Sant Joan de les Abadesas, Ripoll, Torells, etz.

Squalius leuciscus, Heckel et Kner.

Syn.: Leuciscus vulguris, Günth., Cuv. et Valenc., Charvet; Squalius leuciscus, Blanch., Siebold, Gehin; Squalius Bearnensis, Blanch.; Leuciscus Grislagine, Nilsson; Squalius Dobula. CBp.; Leuciscus argenteus, CBp., Agass., Selys-Longchamps, Marcotte; Cyprinus mugilis, Vallot; Cyprinus leuciscus, Bonnat., Lacep., Linn., Bloch; Cyprinus grislagine, Linn., Fries et EKström; Cyprinus dobula, Linn., Bloch; Cyprinus, Arted.; Leuciscus, Bell.

Nom vulgar: Bagre, Vilarodona (Riu Gayá); Madrilla, Xerta, Mora d'Ebre, García (Riu Ebre), Artesa de Segre (Riu Segre) y pobles del Canal d'Urgell, etz.

Especie, encar que prou coneguda, nova per Catalunya. La Bagre de Vilarodona potser siga una Var., car exceptuant el color, pel tamany y la disposició dels raigs de les aletes se sembla a la S. Souffia, Moreau.

Familia dels Cyprinodontidae

Género, CyPRINOdon, Lacep. Cyprinodon iberus, Cuv. et Valenc.

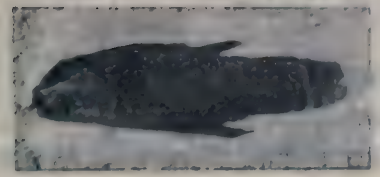

(mascle)

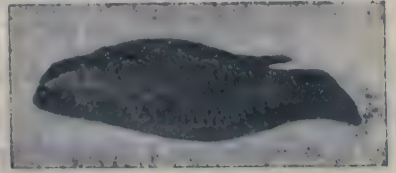

(femella)

Syn.: Fundulus hispanicus.

Nom vulgar: no'n té. Canalissos y rechs de Prat de Llobregat, estanys y aygues salobres de la Marina; estanys y cequies del Recó de Salou. Caldria observar si aquest Cyprinodon ingerit produeix mareig, basca, mal de ventre y vomits y si'ls petits mamifers (gats y gossos) al menjarlo, moren enmatzinats. El Cyprinodon iberus, Cuv. et Valenc. te moltissima ressemblança amb el Fundulus ietourneluxi, Sauvage, que habita's estanys de l'illa de Creta. Te: Br. 4. Anal amb 11 y 12 raigs; coloració olivacea 
amb unes 18 faixes negroses transversals en el mascle y unes faixes negroses longitudinals $o$ en el sentit de la linea lateral en la femella. L'alçaria del cos passa de $3 \frac{1}{4}$ en la femella y 4 en el mascle y la llargaria del cap es de $41_{6}^{1}$ a 5 , respecte la llargaria total que es de 0`036 m. en el mascle y de 0`040 m. en la femella, negrejant solçament l'extremitat de la dorsal y de l'anal en el mascle.

No pot confondres amb las especies Cyprinodon calaritanus, Belloti $y$ C. fasciatus, Martens, car no te'l color de la primera, ni l'anal amb 8 raigs com la segona.

Familia dels Clupeida.

Género, Meletta.

Meletta Phalerica, Moreau.

Syn.. Aphya Phalerica; Rond.; Clupanodon phalerica, Riss.; Meletta mediterranea, Cuv. et Valenc.; Clupea phalerica, CBp.; Alausa papalina, Canestr.

Nom vulgar: Amploia, Meleta, Tarragona; Patoya, Caramelo, la petita o de cria.

No havem vist la Spratella pumila, Valenc.; será segurament la Meletta phalerica, Moreau o M. mediterranea, Cuv. et Valenc.

Género, Sardinella, Valenc.

Sardinella aurita, Cuv, et Valenc.

Syn.: Clupea Maderensis, Günth.; Clupea aurita, Günth.; Engraulis Desmaresti, Riss.; Sardinella aurita, Canestr.

Nom vulgar: Latria, Sardina alatxa, Tarragona; Sardina, Alatxa, Barcelona; Alatxa, Alatxa-pixota, Menorca. Aquest peix salta de l'art com les llisses.

Género. Alosa, Cuv.

Alosa vulgaris, Troschel.

Syn.: Alausa vulgaris, Heckel et Kner, Siebold, Canestr., Cuv. et Valenc., Selys-Longchamps., Guichen., Marcotte, Blanch.; Alausa communis, CBp.; Clupanodon alosa, Riss.; Clupea alosa, Lacep., Riss., Linn., Günth., Schlegel.

Nom vulgar: Alatxu, Saboga, Tarragona; Saboga, Riveres de l'Ébre; Guerxa, Costa de Llevant. 
Especie no citada y que abunda en les aygues de l'Ebre fins a I'açut de Xerta y se la pesca a vegades en el de Flix. Pescat a la roija en la desembocadura del Francoli'l 14 de setembre de 1910, adquirirem un bell exemplar de 0’52 m. de llarch.

\section{Alosa Finta, Marcotte.}

Syn.: Clupea Finta, Günth., Cuv. et Valenc.; Alausa Finta, Blanch., Selys-Longchamps, CBp., Troschel, Siebold; Clupea falla.x, Lacep.; Clupea alosa, Bloch.

Nom vulgar: Alatxa, Tarragona; Saboga, Nlosa, Barcelona; Saboga, Riveres de l'Ebre; Guerxa, Costa de Llevant.

Mes tardana que l'anterior abunda en les aygues del mateix riu.

La Llampresa (Petromyzon fluviatilis, Blaiv.), ataca les sabogues. La saboga llampresada no te l'estima de l'altra, car está mes magra y la seva carn no te tan bon gust.

\section{Alausa Pilchardus, Cuv, et Valenc.}

Syn.: Clupea sardina, CBp., Cuv.; Alausa pilehardus, Marcotte, Guichen., C. Millet; Clupea pilchardus, Cuv., Arted., Bloch, CBp., Günth.; Clupea spratus, Brunn.; Harengus minor seu pilchardus, Willugh.; Sardina, Bell, Cetti, Rond., Duham.,Bonnat.

Nom vulgar: Sardina, Sardineta, Patoya, Caramelo, la petita o de cría, Tarragona: Sardina, Barcelona; Sardina,. Junqueti, la petita o de cria, Menorca.

Abunda molt. Pels mesos d'Abril, Maig y Juny, els arts y els sardinals n'agafen segons els dies, en un sol vol mes de 1.000 kilograms. Part d'aquest peix, després de Sant Joan Baptista 's torna cego. d'aquí ve la dita dels sardinalers: d'armar per anar a la cega o a les cegues. La ceguera no creyem que siga cap constelació epidémica, ni cap congestió oftálmica ocasionada pels esforços que fa'l peix al veures enmallat, ja que es quan la sardina va a les acaballes, es a dir, quan es mes grossa y está mes grassa. Probablement es una oftalmia parassitaria produhida per un crustaci parassit de la fa milia dels Lernaeidae.

Género, Engraulis, Cuv.

Engraulis encrasicholus, CBp.

Syn.: Engraulis encrasicholus, Günth., Schlegel, Canestr., Cuv. 
Cuv. et Valenc., Riss., Selys Longchamps, Guichen.; Engraults Meletta, Cuv., CBp.; Clupea encrasicholus, Lacep., Riss., Bonnat., Linn., Bloch, Brunn.

Nom vulgar: Aladroch, Seitó, Tarragona; Seitó, Barcelona; Aladroch, Menorca. Abunda molt y se'n prepara l'anxova, industria de certa importancia en determinades localitats de la costa catalana.

Familia dels Exocaetidae.

Sub-familia dels Belonini.

Género, BELONE, Cuv.

Belone vulgaris, Cuv. et Valenc.

Syn.: Belone rostrata, Faber, CBp.; Belone vulgaris (Orphia vulgar), Marcotte; Belone vulgaris (Orphia comuna), Selys-Longchamps, Günth., Schlegel; Esox belone, Lacep., Bonnat., Linn., Bloch, Rosenthal.

Nom vulgar: Agulla, Barcelona, Tarragona; Agua, Menorca.

Belone acus, Riss.

Syn.: Belone acus, CBp., Günth., Canestr.; Belone acus, (Orphia agulla) Riss., Cuv. et Valenc., Guichen.; Esox belone, Riss., Brunn.

Nom vulgar: Agulla, Barcelona, Tarragona; Agua, Menorca.

D'aquesta y de l'anterior especie d'Agulles se'n agafen grans cantitats amb l'agullera (Art especial).

\section{Belone imperialis, Moreau.}

Syn.: Belone cantrainii, Günth., Cuv. et Valenc.; Tylosurus imperialis, CBp., Canestr.; Tylosurus cantrainii, Cocco; Eso.t imperialis, Rafin.

Nom vulgar: Agulla, Tarragona. Especie nova per Catalunya.

Pescat al llum el 5 de Juliol de 1910 n'adquiriren un bon exemplar de 1200 grams de pes y $0990 \mathrm{~m}$. de llarch.

Sub familia dels Exocaetidae.

Género, ExocaEtus, Linn. Exocaetus volitans, Linn.

Syn.: Exocaetus volitans, Cuv. et Välenc., Guichen., Günth., 
Canestr., Lutken; Exocaetus exiliens, Bloch, CBp.; Hirundo, Salvian.

Nom vulgar: Barat volador, Tarragona; Uranyola, Barcelona•

\title{
Exocaetus Evolans, Linn.
}

Syn.: E.rocaetus obtusirostris, Günth., Lutken; Exocaetus evolans, Cuv. et Valenc., Bloch, CBp., Günth., Canestr., Lutken, Bonnat.; E.zocaetus volitans, Lacep., Riss.

Nom vulgar: Barat volador, Barcelona, Tarragona; Oronyola, Menorca.

\author{
Familia dels Scopelidae. \\ Subfamilia dels Scopelini. \\ Género, Scopelus, Cuv. \\ Scopelus Pseudo crocodilus, Moreau.
}

Syn.: Scopelus resplandens, Gigl.; Scopelus caudispinosus, Gigl.; Scopelus elongatus, Giglioli, Steindachn., Vinciguerra. Belloti; Scopelus crocodilus, Cuv. et Valenc.

Nom vulgar: Maire d'Amplova, (Catalunya francesa.) Especie nova per Catalunya.

Pel pescador del lloch de Clará (Torredembarra) en Pau Mercader, reberem de Calafell pel recader de Tarragona a Barcelona, pescats en alta mar, el 15 de Juny de 1910, els dos únichs exemplars que havem vist y que serva l'Institució Catalana de Historia Natural.

Ofereixen la particularitat, ademés dels caracters que'ls assignen els autors, de presentar un punt daurat en quiscuna de les branquies del maxilar superior casi a la mateixa sínfisis, com el del Scopelus Humboldti, Riss.

\section{Género, Maurolicus, Cocco. Maurolicus Poweriße, Günth. .}

Syn.: Scopelus Poweria, Cuv. et Valenc.; Gonostomus Powe. ria, Cocco; Ichthyococus Powerix. CBp.; Maurolicus Powerix, Canestr., Giglioli.

Nom vulgar: No'n té. Especie excesivament rara y nova per Catalunya, citada com a marítima y viu en les aygues salmástigues de l'estany y cequies del Recó de Salou. Mideix fins á l'arrancada de la 
cua 0‘030 m.: el cap 0‘009 m. Te dugues dorsals, la primera comensa al bell mitg del cos, amb 6 raigs y la segona amb 11 ó 12 . Les ventrals comensen alli hont acaben les pectorals y l'anal amb 11 ó 12 raigs alli hont acaben les ventrals.

Género, Saurus, Cuv.

Saurus fasciutus, Riss.

Syn.: Saurus lacerta, Cuv. et Valenc., Guichen., CBp., Ca nestr.; Saurus trivirgatus, Valenc.; Saurus griseus, Lowe; Saurus mediterraneus, Costa; Osmerus fasciatus, Riss.; Osmerus saurus. Rafin., Lacep.; Salmo saurus, Bonnat., Linn.; Suurus, Salvian. Willugh.

Nom vulgar: Dragó, Barcelona, Tarragona, Menorca.

Género, Aulopus, Cuv. Aulopus filamentosus, CBp.

Syn.: Aulopus filamentosus, Cuv. et Valenc., Guichen., Günth., Costa, Canestr.; Aulopus maculatus, (f.), Valenc.; Aulopus filifer (m.), Valenc.; Saurus lacerta, Lowe, Riss.; Osmerus lacerta, Riss.; Salmo tirus, Rafin.; Salmo filamentosus, Bloch.

Nom vulgar: Lluert, Torredenbarra; Salte muradas, Serpenti, Escalum, Menorca.

Aulopus Agassizi, Vaill.

Syn.: Chlorophthalmus Agassizi, CBp., Costa, Günth., Canestr., Giglioli; Aulopus Agassizi, Cuv. et Valenc.

Nom vulgar: No'n té. Especie excessivament rara y nova per Catalunya.

El 18 de Febrer de 1913 adquirirem els tres únichs exemplars que havém vist y serva l'Institució Catalana d'Historia Natural.

Familia del Salmonida.

Género, SALmo.

Sub-género, SALmo.

Salmo salar, Linn.

Syn.: Salmo salar, Marcotte, Blanch., Soland, Lacep., Fre. Oge rien, Bonnat., Cuv., Vallot, Bloch, Rosenthal, Nordmann, Selys- 
Longchamps, CBp., Hecker et Kner, Nilsson, Günth., Gehin, Schlegel; Trutta salar, Siebold: Salmo Salmo, Cuv. et Valenc.; Salmo, Yarr, Couch; Salmo vel Sulmo, Bell.

Nom vulgar: Salmó, Tarragona.

En les aygues del port de Tarragona el 9 de Maig de 1910 pescaren a la roija un Salmó de 5'50 kilograms que compraren els PP. Cartoixos de la Chartreuse; es l'única o una de les poques vegades que pot citarse aquesta especie en el mediterrá. Probablement seguiría l'estela del vapor anglés Badebev que carregat de carbó mineral hi fondejá'l dia avans procedent de Swansea.

La carn del Salmó es en els mercats la mes cara de totes pagantse a voltes a preus fabulosos.

\section{Salmo Fario, Linn.}

Syn.: Trutta variabilis, Lunel; Trutta fario, Blanch., Siebold. Canestr., Gehin; Trutta lacustris, Blanch.; Salar Ausonii, Cuv. et Valenc., Heckel et Kner, Marcotte; Fario Lemanus, Cuv. et Valenc.; Salmo trutta, Agass., Lacep., Riss., Bonnat., Jurine; Salmo fario, Cuv., Vallot, Riss., Crespon, Bonnat., Bloch, Selys Longchamps, Agass., CBp., Günth., Schlegel; Trutta fluviatilis, Duham.

Nom vulgar: Truyta: truyta salmonada la de carn roija. Rius Ebre, Noguera Ribagorçana, Noguera Pallaresa, Segre, Fresser, Ter, Estanys del Pallars, de Carança, etz.

El Director de l'Aquarium y Labol atori ictiogénich del Park de Barcelona en Francesch d'A. Darder, ha introduit en les aygues dolces de qualques indrets de Catalunya, a mes del Salmo levenensis, G. (Salmó d'Escocia), el (Salmo) fontinalis, Salvelinus fontinalis, Mitchil, (Salmó de California, Brootk Tront) y'l Salmo iridens, Gibbons, (Salmó Arch de St. Martí d'América). Ignorém els resultats d'aquestes tentatives, més es probable que la repoblació tingui éxit, car son salmónits que viuhen fins a termalitats de $24^{\circ}$, pero no deu per aixó descuidarse la conservacio de les especies que viuhen naturalment en nostres aygues.

Género, Argentina. Argentina sphyraena, Linn.

Syn.: Goniosoma argentinum, Costa; Argentina Cuvieri, Cuv. 
et Valenc.: Argentina sphyraena, Cuv., Lacep., Riss., Bonnat., Brunn., CBp., Günth., Canestr.; Argentina, Duham., Willugh.

Nom vulgar: Moixó, Tarragona. Peix de fonera conegudissim pero encara no citat.

No tenim noticies que s'aprofiti, com se fa en altres paysos, el pigment argentat de la bufeta natatoría y que recubreix el cos d'aquesta especie, anomenat (Essencia d'Orient) que s'emplea en la fabricació de perles falses, lo mateix que's fa amb la materia argentífera que revesteix la part de dintre de les escates de la Breca (Alburnus lucidus, Heckel et Kner).

Ordre dels APODEs.

Familia dels Anguillidae.

Género, Anguilla. Anguilla acutirostris, Kaup.

Syn.: Anguilla vulgaris, CBp., Costa, Siebold, Canestr., Gehin, Schlegel, Günth., C. Dareste, Blanch., Soland, Lunel, Cuv., Marcotte: Anguilla fluviatilis, Agass., Heckel et Kner; Anguilla medio rostris, Blanch., Riss.; Angnilla oblongirostris, Blanch.; Anguilla acutirostris, Riss., Selys-Longchamps, Blanch.; Anguilla oxvcephala, De la Pylaie; Anguilla, Bell.; Murena anguilla, Lacep., Riss., Crespon, F.re Ogerien, Linn., Bloch, Jurine, Vallot.

Nom vulgar: Anguila, Barcelona, Tarragona, Lleyda y Girona: Anguila vera, Menorca.

L'anguila no's troba en el riu Ter ans de l'ayguabarreig de Ripoll, ignorem la causa, però'ns sembla que mes será per la mancansa de llot que per les baixes temperatures y mineralisació de les aygues.

Var. Anguilla latirostris, Kaup.

Syn.: Anguilla macrocephala, De la Pylaie; Anguilla Cloacina, CBp.; Anguilla Cuvieri, Kaup; Anguilla latirostris, Blanch., Selys-Longchamps, Günth., Riss.

Nom vulgar: Anguila de mar, Barcelona, Tarragona; Anguila cabotera, Menorca.

Var. Anguilla' microptera, Kaup.

Nom vulgar: Anguila, Tarragona; Anguila roija, Menorca.

Var. Anguilla capitone, Kaup.

Nom vulgar: Anguila, Tarragona; Anguila punyegral, Menorca. 
S'han trobat anguiles d'aygua dolça, fetes 0 adultes, transparents. o mancades de pigment, particularitat deguda a un desenrotllament incomplert y a servar els caracters de cuch o larva. Aquestes anguiles no poden constituhir una nova especie, car aixó es propi del medi o mes be d'un dels quatre medis en que s'agita la vida soterrania, amb analogies, així com diferencies amb les cavernes propiament dites y amb llurs aygues corrents o estancades.

El descoloriment o despigmentació dels ulls, de la pell y dels pels per la mancansa de pigment (ocular, cutani, pilós) o materia orgánica particular colorada en negre o en roig, es un dels caracters propis dels animals soterranis y no pot fins al present explicarse; evidentment prové de l'absencia de la llum, de l'afebliment d'absorció dels raigs lluminosos que suprimeixen certes reaccions fisiológiques, peró s'ignora per quin fenómen exacte's produheixen.

Género, Conger, Cuv.

Conger vulgaris, Cuv:

Syn.: Conger vulgaris, CBp., Kaup, Günth., Schlegel, Canestr., Selys-Longchamps, Guichen., Marcotte; Conger verus, Riss.; Muraena conger, Lacep., Riss., Bonnat., Linn., Bloch; Conger, Rond., Bell., Salvian.; Muraena supremo margine pinnae dorsalis nigra, Arted.

Nom vuigar: Congre blanch, Congre de fang, Tarragona; Congre, Barcelona, Menorca.

\section{Var. Conger niger, Kaup.}

Syn.: Muraena nigra, Riss.; Conger niger, Riss., Guichen., CBp.

Nom vulgar: Congre negre, Congre d'alguer, Congre de roquer, Congre de forat. Tarragona.

\section{Leptocephalus Morrisii, Lacep.}

Syn.: Leptocephalus Spallanzani, Kaup, Riss.; Leptocephalus pellucidus, CBp., Canestr., Riss.; Leptocephalus candidissimus, Costa; Helmictis punctatus, Rafino; Leptocephalus Morrisi, BI. Schneid., CBp., Kaup, Günth.; Leptocephalus lineatus, Bonnat. 
Nom vulgar: Congret, Tarragona; Flamula, Menorca. Es el congre petit o de cria.

N'havem examinat moltissims. Tots de $0^{\prime} 12 \mathrm{~m} . \times 0^{\prime} 006 \mathrm{~m}$. de llargaria y alçaria; tenen .'iris argentat y una senyal unguicular negra en la part superior de l'órbita; en la linea lateral, de dos en dos $o$ de tres en tres, presenten una serie de puntets negres que també se'Is hi veuhen a un costat y altre del dors y del ventre; no se'ls coneixen les aletes, pero en el punt corresponent a les pectorals, la pell está com una mica alçada.

S'e'l troba barrejat amb el Caramelo (Meletta phalerica, M., Alausa Pilchardus. Cuv. et Valenc.) y no l'havem vist indicat en -cap Catálech de peixos que's refereixhi a Catalunya.

\section{Conger Balearicus, CBp.}

Syn: Conger muraena balearica, Günth., Kaup: Conger balearicus, C. Dareste; Gonger Cassini, Riss., Guichen.; Muraena Cassini, Riss.; Muraena balearica, Delaroche.

Nom vulgar: Congre. Congre serpeté, Tarragona. Especie nova per Catalunya.

\section{Conger Mysta.t, Riss.}

Syn.: Congromuraena Mystax, Günth.; Conger muraena mystax, Kaup; Ophidium imberbe, Riss.; Conger mysta.x, CBp., Canestr.; Muraena mysta.x, Delaroche.

Nom vulgar: Congre Serpeté, Tarragona; Pillet de platja, Con gre dolç o de sucre, Menorca.

\section{Familia dels Myridae. \\ Género, Myrus, Kaup. Myrus vulgaris, Kaup.}

Syn.: Conger myrus, Costa, CBp., Canestr., Riss., Cuv.. Gui . chen.; Myrus vulgaris, Günth.; Muraena myrus, Lacep., Riss., Bonnat., Linn., Brunn.; Serpens marinus alter cauda compressa. Willugh.; Muraena rostro acuto, lituris albidis vario, margine pinnae dorsalis nigro, Arted.

Nom vulgar: Serp, Congre serpeté, Barcelona, Tarragona; Culebra, Duradu, Menorca. 


\section{Familia dels Muraenidae. \\ Género, Murena. \\ Muraena Helena, Linn.}

Syn.: Muraena, Couch, Bell., Salvian., Willugh., Rond.; Muraena Helena, Guichen., Cuv. et Valenc., Riss., Bonnat., Brunn., Bloch, Rafin.,Rosenthal, CBp., Costa, Kaup, Günth., Canestr.; Mu ruena guttatu, Riss.; Muraena fulva, Riss.; Muraenophis fulva, Riss.; Muraenofis helena, Riss.; Muraena pinnis pectoralibus carens, Arted.

Nom vulgar: Murena, Barcelona, Tarragona, Menorca.

\section{Muraena unicolor, Cuv.}

Syn.: Muraenophis unicolor, Delaroche; Muraenophis Cristini, Riss.; Muraena Cristini, Riss.; Muraena unicolor, Guichen.; CBp., Günth., Canestr.; Thyrsoidea unicolor, Kaup.

Nom vulgar: Murena, Barcelona, Tarragona; Muret, Menorca, Rara.

Les mossegades de les Murenes son tan fortes, segons la gent de mar, que arriven a deixar senyals en el ferro y altres metalls.
Familia dels Ophisurida.
Género, OpHIsurus, Lacep.
Ophisurus Serpens, Lacep.

Syn.: Ophichthys serpens, Günth.; Ophisurus serpens, CBp.. Costa, Kaup, Canestr., Riss., Guichen.; Muraena exacte teres, cauda acuta apterygia, Arted.; Serpens marinus, Bell., Salvian., Willugh., Rond.

Nom vulgar: Serp de mar, Barcelona, Tarragona; Culebra de la mar, Menorca.

\section{Ophisuras hispanus, Belloti.}

Syn.: Ophisurus hispanus, Günth.; Ophichthys hispanus, Giunth.

Nom vulgar: Serp de mar, Tarragona. Especie nova per Catalunya. 
Familia dels Sphagebranchido.

Género, Sphagebranchus, Bloch. Sphagebranchus imberbis, Delaroche.

Syn.: Ophichthys imberbis, Günth.; Leptocephalus Spallanzani, Riss.; Sphagebranchus imberbis, Riss., Costa, CBp., Kaup, Canestr.; Sphagebranchus oculatus, Riss.

Nom vulgar: Serp de mar, Tarragona, Menorca; Cuca serp, Bar. celona.

Sphagebranchus cacus, Bl. Schneid.

Syn.: Ophichthys coecus, Günth.; Apterichthys coecus, Kaup, Delaroche; Sphagebranchus Spallanzani, Costa; Sphagebranchus serpa, Riss.; Sphagebranchus bimaculatus, Riss.; Sphagebranchus coecus, Riss., CBp., Canestr.; Coecilia Branderiana, Lacep., Rafin.; Muraena coeca, Linn., Bonnat.

Nom vulgar: Serp de mar, Barcelona, Tarragona; Murer, Menorca.

L'atrofia íncomplerta dels organs visuals que presenta l'especie S. coecus, Bl. Schneid, es un del caracters afecte a les bestioles soterranies y crepusculars y lo mateix que'l nostre Gammarus puteanus, Döflein de la cova dels Hams (Illa de Mallorca), modificació adaptiva del G. Pulex, Lam, en les cavernes, per adaptació provocada per la pressió, la temperatura, la nudrició, la fosca, etz. Tam. bé's troba en els animals dels abims o profonditats de la mar. I en compliment de la lley natural de compensació o excitació funcional d'en Lamarck o"l principi d'equilibri dels organs d'en Geoffroy-Saint Hilaire deu presentar hipertrofia o extensió considerable dels organs del tacte, de l'olfat y de l'oido.

Sub-classe dels Marsipobranchis.

Ordre dels Ciclostómits.

Sub-ordre dels Petromyzons.

Familia dels Petromyzonidae.

Género, Petromyzon; Arted.

Petromyzon marinus, Linn.

Syn.: Petromyzon marinus, Cuv., Born, Vallot, Riss., Blain- 
ville, Marcotte, Blanch., Soland, Lacep., Bonnat., Bloch, SelysLongchamps, CBp., Nilsson, Heckel et Kner, Sièbold, Canestr., Bert, Gehin, Günth., Schlegel: Mustela sive Lampetra, Bell., Salvian., Willugh.; Petromyzon maculosus, etz., Arted.

Nom vulgar: Llampresa de mar, Barcelona, Tarragona; Ferretimò, Xuclador, Menorca.

Petromyzon fluviatilis, Linn.

Syn.: Pétromyzon argenteus, Bloch, Lacep., Couch; Petromyzon sep-aeil, Lacep.; Petromyzon niger, Lacep.; Petromyzon fluviatilis, Cuv., Blainville, Marcotte, Blanch., Soland, Lacep., Bonnat., Bloch, Selys-Longchamps, CBp., Nilsson, Heckel et Kner, Siebold, Canestr., Gehin, Günth., Schlegel; Lampetra parva et fluviatilis, Willugh.; Petromyzon, Arted.; Lampetra, Salvian.; Mustela fluviatilis minor, Bell.

Nom vulgar: Llampresa de riu. General.

\section{Petromyzon Planeri Marcotte.}

Syn.: Petromyzon Planeri, Bloch, Selys-Longchamps, CBp., Nilsson, Heckel et Kner, Günth., Siebold, Canestr., Bonnat., Lacep., Omalius de Halloys, Cuv., Blainville, Marcotte, Blanch.; Petromızon sanguisuga, Lacep.

Nom vulgar: Llampresa petita o marina, Tarragona. Especie nova per Catalunya.

Ammocoetes branchialis, Linn.

Syn.: Petromyzon ruber, Lacep.; Ammocoetus, C. Dumeril; Lampetra coeca, Willugh; Petromyzon branchialis, Bloch, Günth., Bonnat., Lacep., Omalius de Halloys; Ammocoetes branchialis, Selys-Long champs, CBp., Nilsson, Marcotte.

Nom vulgar: Cuch de gleva, Clapissá, Tarragona. No l'havem vist citat en cap catàlech que's refereixi a Catalunya y es prou conegut dels nostres palangrers y aficionats a la pesca, car se'l considera com a un dels millors esquers.

Es la larva o cuch del Petromyzon que moltes vegades se troba a les platjes formant bolichs sobre l'Asperococcus compressus, 
Griffith (Coleta) alga morena de la familia de les Pheospóreacees escupida pel mar.

Sub-classe dels Pharyngobranchis.

Ordre dels Amphioxienchs.

Familia dels Branchiostomidoe.

Género, Branchiostoma, Costa.

Amphioxus lanceolatus, Yarr.

Syn.: Amphioxus lanceolatus, Kolliker, Sundewall, Goodsir, Quatrefages, Kowalewsky, P. Bert, E. Moreau, Huxley; Branchiostoma lanceolatum, Costa; Branchiostoma lubricum, Costa, J. Müller, Marcůsen, Canestr.; Limax lanceolatus, Pallas.

Nom vulgar: no'n te. Especie nova per Catalunya; abunda en el Golf de Rosas.

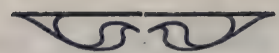




\section{ADDENDA}

Familia dels Trygonits. Género, Pteroplatea, Müll. et Henl. Pteroplatea Altavela, Müll, et Henl.

Syn.: Trygon Altavela, CBp.; Pteroplatea canariensis, Guichen.; Pteroplatea altavela, CBp., Bruch, A. Dumer., Günth., Canestr., Brit. Capel., Giglioli, Doderl., Perugia; Dasybatis altavilla, Rafin.; Raia pastinaca altavela, Bonnat., Lacep.; Raia altavella, Linn.; Aquila autoris Prior, Aldrov.; Pastinaca marina altera, altavela dicta, Willughb.

Nom vulgar: Vaca, Barcelona; Milá, Totina, Tarragona. Especie nova per Catalunya.

N'havem vist pochs exemplars. Pel Desembre de 1905, en Amador Romaní y Guerra, de Capellades, en veigé un exemplar en casa Lluis Soler, naturalista preparador de Barcelona, quals dimensions eren: 0 ‘ $52 \mathrm{~m}$. de llargaria $\times 0$ 0'99 m. d’amplaria.

Familia dels Bleniidae.

Género, Blennius, Arted.

Blennius Basiliscus, Valenc.

Syn.: Blennius Basiliscus, Günth., Canestr., Cuv. et Valenc.; Ichthyoris Basiliscus, CBp.

Nom vulgar: Dormilega, Tarragona. Especie rara y nova per Ca. talunya. En 30 de Janer de 1913 ne recullirem dos exemplars.

Familia dels Callionymidae.

Género, Callionymus, Linn.

Callionymus Phaeton, Günth

Syn.: Callionymus Morisonii, CBp.; Callionymus festivus, CBp., Canestr.; Callionymus Phaeton, Giglioli, Vaill. 
Nom vulgar: Guineu vermella, Tarragona. Especie nova per Catalunya.

En 20 de Febrer de 1912 ens vingueren á les mans dos exemplars mascles: l'un de 0 '15 m. y l'altre de $0.12 \mathrm{~m}$. qui presentaven la particularitat de que l'allargament de llur preopercul en lloch d'estar armat de tres punxes, no mes ne tenia dugues, l'una dirigida cap a dalt y endevant y l'altra no mes cap á dalt.

El 28 de Janer de 1913 recullirem un exemplar de Trigla cavillone, Lacep., amb dos geps, un al començament de la dorsal y altre á l'acabament.

El 24 de Febrer de 1913 tinguerem el goig de veurer pescats al bou á Tarragona, tot un vol de Hoplostethus mediterraneus, Cuv. et Valenc., peró tenien la dorsal amb $7 / 14$ raígs y l'anal 3/11 y no amb 6/12 ó 13 y $3 / 10$ raigs y'ls bucliers de la base de les ventrals á á l'Anus, com la major part de les Clupees, eren en número de 8 , no en número variable, car n’hi havía de $0^{`} 12 \mathrm{~m}$., 0`15 m. y ()‘18 de llargaria y totes presentaven 8 bucliers.

\section{Familia dels Gasterosteidce.}

Género, Gasterosteus, Linn.

Gasterosteus semiloricatus, Cuv. et Valenc.

(Var. del Gasterosteus aculeatus, Linn.)

Syn.: Gasterosteus Neustrianus, Blanch., Sauv.; Gasterosteus semiloricatus, Blanch., Soland, CBp., Günth.

Viu en companyia del Cyprinodon iberus, Cuv. et Valenc. y del Maurolicus Poweriæe, Günth. en les aygues salmástigues de l'estany y cequies del Recó de Salou. (1)

(1) Amb el llarch temps que ha durat la publicació de aquest trevall en el «ButIletí de l'Institucic Catalana d'Historia Natural', son moltes mes que les indicades en el Prólech, les especies y varietats noves, y aixís mateix pel motiu de publicarse en plechs á part. no havem pogut adoptar les noves normes ortográfiques com hauria sigut nostre desitg. 


\section{INDEX}

\section{dels noms vulgars}

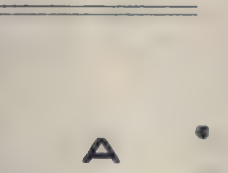

Págs.

Págs.

Abadeco . . . . . 40-41 Anguila roija. . . . . . 90

Abadeco ratllat. . . . . $\quad$ 38-39 Anguila vera. . . . . . . 90

Agua. . : . . . . . 86 Aranya. . . . . . . 26

Agulla. . . . . . 23-86 Aranya blanca . . . . . 26

Agullat. . . . . . . 15 Aranya capsuda. . . . . 26

Agullat xato. . . . . . 15 Aranya de capnegre . . . 26

Aladroch . . . . . . 85-86 Aranya fragata. . . . . . 26

Alatxa. . . . . 84-85 Aranyò. . . . . . . 26

Alatxa pixota. . . . . . 84 Arbecona. . . . . . 43-44

Albecora . . . . . . 43-44 Armat. . . . . . . . 33

Alosa . . . . . . . . 85 Ase . . . . . . . 34-67

Amphioxus . . . . . . 96 Ase mossegayre. . . . . 28

Amploia . . . . . . . 84 Aseta . . . . . . . . 67

Anfós . . . . . . 38-41 Aulopus. . . . . . . . 88

Angel . . . . . . . . 16 Auruda. . . . . . . . 55

Anguila . . . . . . 90 Auradella. . . . . . . 55

Anguila de mar. . . . . 90 Auradeta . . . . . . 52

Anguila punyegral. . . . 90

\section{B}

Bacoreta . . . . 4 43-44 Baldufa. . . . . 4 43-44

Bagre . . . . . . 83 Ballesta. . . . . . . . 25

Baila . . . . . . 37 Barada . . . . . 51

Bairó . . . . 45-46 Barb. . . . . 80-81 
Nom vulgar: Guineu vermella, Tarragona. Especie nova per Catalunya.

En 20 de Febrer de 1912 ens vingueren á les mans dos exemplars mascles: l'un de 0'15 m. y l'altre de 0.12 m. qui presentaven la particularitat de que l'allargament de llur preopercul en lloch d'estar armat de tres punxes, no mes ne tenia dugues, l'una dirigida cap a dalt y endevant y l'altra no mes cap á dalt.

El 28 de Janer de 1913 recullirem un exemplar de Trigla cavillone, Lacep., amb dos geps, un al començament de la dorsal y altre á l'acabament.

El 24 de Febrer de 1913 tinguerem el goig de veurer pescats al bou á Tarragona, tot un vol de Hoplostethus mediterraneus, Cuv. et Valenc., peró tenien la dorsal amb 7/14 raígs y l'anal 3/11 y no amb 6/12 ó 13 y 3/10 raigs y'ls bucliers de la base de les ventrals á á l'Anus, com la major part de les Clupees, eren en número de 8, no en número variable, car n’hi havía de 0‘12 m., 0`15 m. y ()'18 de llargaria y totes presentaven 8 bucliers.

Familia dels Gasterosteida.

Género, Gasterosteus, Linn.

Gasterosteus semiloricatus, Cuv. et Valenc.

(Var. del Gasterosteus aculeatus, Linn.)

Syn.: Gasterosteus Neustrianus, Blanch., Sauv.; Gasterosteus semiloricatus, Blanch., Soland, CBp., Günth.

Viu en companyia del Cyprinodon iberus, Cuv. et Valenc. y del Maurolicus Powerioe, Günth. en les aygues salmástigues de l'estany y cequies del Recó de Salou. (1)

(1) Amb el llarch temps que ha durat la publicació de aquest trevall en el «Butlletí de l'Institució Catalana d'Historia Natural», son moltes mes que les indicades en el Prólech, les especies y varietats noves, y aixís mateix pel motiu de publlcarse en plechs á part, no havem pogut adoptar les noves normes ortográfiques com hauria sigut nostre desitg. 


\section{INDEX}

\section{dels noms vulgars}

$A$

Págs.

Págs.

Abadeco . . . . . . 40-41 Anguila rcija. . . . . . 90

Abadeco ratllat. . . . $\quad$ 38-39 Anguila vera. . . . . . . 90

Agua. . . . . . . . . 86 Aranya. . . . . . . . . 26

Agulla. . . . . . 23-86 Aranya blanca . . . . . 26

Agullat. . . . . . . 15 Aranya capsuda. . . . . 26

Agullat xato. . . . . . . 15 Aranya de capnegre . . . 26

Aladroch . . . . . . 85-86 Aranya fragata. . . . . 26

Alatxa . . . . . . 84-85 Aranyò. . . . . . . 26

Alatxa pixota. . . . . . . 84 Arbecona . . . . . . 43-44

Albecora . . . . . . 43-44 Armat. . . . . . . . 33

Alosa . . . . . . . 85 Ase . . . . . . . 34-67

Amphioxus . . . . . . . 96 Ase mossegayre. . . . . . 28

Amploia . . . . . . . . 84 Aseta . . . . . . . . . 67

Anfós . . . . . . 38-41 Aulopus. . . . . . . 88

Angel . . . . . . 16 Auruda. . . . . . . 55

Anguila . . . . . . 90 Auradella. . . . . . . 55

Anguila de mar. . . . . . 90 Auradeta . . . . . . . . 52

Anguila punyegral. . . . . 90

Bacoretæ. . . . . 43-44 Baldufa. . . . . . 43-44

Bagre . . . . . . . 83 Ballesta. . . . . . . 25

Baila . . . . . . 37 Barada. . . . . . . 51

Bair6 . . . . . 45-46 Barb. . . . . . . 80-81 
Barb caní. . . . . . 27

Barat. . : . . . . 43

Barat d'ull gros _ . . . 43

Barat volador .. . . . 86-87

Bastina . . . . . 9

Bavosa . . ... 27-28

Bestenaga . . . . . . 59

Besuch $\cdots, \ldots$

Beta $\quad=50$

Biret

Biró.
34

$45-46$
Bissu .

43

3 Boca dolca. . . . . . 14

3 Boga.

Bogaravell . . ? . . 54

Bogarró . . . . . . 52

Bonito.

Bonitol

44-45

Borratxo .

44-45

Bot. . . . . . . 25

Buldroi .. . . . . . 30

Burru
Cabessuda

Cabot .

Cabot Berrubes.

Cabot d'alga

Cabot de roca .

Cabot en banyes

Cabot inglés.

Cabot mucós.

Cabot xuclador.

Caçó

Caluga.

Camarí.

Canari.

Cántara

Capellá

Capsech

Capsigrany

Cap roig.

Capsut

Captinyós

Caputxo .

Caramelo.

Caramelo ros

Cardaire.

Cardenal

Caro

Carpa carassina.
67

31

32

30

31

29

31

30

79

13

66

11-13-14

61

55

68-78

34

27-28

35

60

36

19

84-85

32

19

51

22

81
Carpa de Bohemia.

Carpa de Franconia

Carpa d'Holanda .

80

Carpa del Japó.

Carpa de Turinga. 48 64 43

Clavell. 19-20-21

Clavellada. 19

Clavell Borrell. 95 Clapissá 34

Clavilló. : . : : : 34-35

Columida. : . . . 46

Congre . . . . . 92

9 Congre dolç. . . . . 68-92

5 Congre blanch. . . . . : 91

Congre d'alguer . . . . . 91

Congre de fang . . . . . . : 91

Congre negre . . . . . . . 91

Congre de forat . . . . . 91

Congre de sucre . . . . ... 92 
Congre de roquer. . . . . 91 Corvina . . . . . . . . 42

Congre serpeté . . . . . 92 Cuca serp . . . . . . . 94

Congret . . . . . . . 92 Cuch de gleva. . . . . . 95

Corcovada . . . . . . 56 Culebra . . . . . . . 92

Cornuda . . . . . . . 13 Culebra de la mar. . . . . 93

Corra . . . . . . . 42 Cullereta. . . . . . . 35

Corvall . . . . . . 42 Cyprinodon. . . . . . . 83

Dentó . . . . . . . 41 Dormilega de cap roig. . . 28

Dento . . . . . . . . 55 Dormilega de fang. . . . 27

Dentol . . . . . . 55 Dormilega de roca : . . 28

Donzella . . . . . . . 63-64 Dormilega roija . . . . . 27

Donzelleta . . . . . . 62 Dot. . . . . . . . 37

Doradu . . . . . . 49-92 Dormilega petita . . . . "28

Dormileg̊ . . . . 27-23-A Dragó. . . . . . 26-29

Enfú . . . . . . . . 67 Esparrai . . . . . . . . 52

Emperador . . . . . 17-49 Esparrall. . . . . . 52

Escalúm . . . . . . . 88 Esparralló. . . . . . . 52

Escat . . . . . . . . 16 Espet . . . . . . . . . 67

Escat vegigal . . . . . . 16 Escrita. . . . . . . . 20

Escatós . . . . . . 35 Escrita blanca. . . . . . 19

Escolá. . . . . . . 71 Escrita vera. . . . . 20-21

Escorpora dels bruts. . . . 35 Escurbai. . . . . . . . 42

Escorpora de fang . . . . 35 Escursana. . . . . . . . 21

Escorpora groga . . . . . 35 Escursó . . . . . . . . 21

Escorpora de roquer. . . 35-36 Esturí6 . . . . . . . . 22

Faixs . . . . . . . 51 Fleuma . . . . . . 51

Ferrassa . . . . . . 21 Fura . . . . . . . 71

Ferre pedres . . . . . . 29 Fura blanca. . . . . . . 72

Ferre timó . . . . . . . 95 Fura negra . . . . . . . 72

Fi de murena mansa. . . : 68 . Futarra . . . . . . . 27-28

Flamula ... . . . 50-92 


\section{G}

Págs.

Págs.

Galta roig . . . . . 65 Gernera . . . . . . 40

Gall. . . . . . . . 47 Gobit . . . . . 30-31

Gall de St. Pere ..... . . 47 Gobit d'alga. . . . . . 30

Gallerbu . . . . . . 27 Gobit de roca. . . . . . 30-31

Gallimó . . . . . . . 43 Golfás. . . . . . . 44

Gallinetə. . . . . . 33 Gripau. . . . . 62-63

Gallet. . . . . , . 48 Griva . . . . . . . 58

Garneu . . . . . . 34 Grivia. . . . . . . 58-59

Gasterosteus . . . . . . A Grivieta . . . . . . . 62

Gat . . . . . . 9-13 Guardia civil . . . . 13

Gatneu . . . . . 34 Guerxa. . . . . 84-85

Gatneu futaire. . . . . 34 Güiula . . . . . 63-64

Gató. . . . . . . . 9 Guineu . . . 22-29-72

Gatvaire. : . . . , 10 Guineu vermella . . . 29-A

Gat ver .. . . . . . 9 Gutxu... . . . . 15

Gerna. . . . . . . 40

\section{$\mathbf{H}$}

Hoplostethus

36 Inflaconys

Jerret. . . . . . . . 57 Junqueti. . . . . . 85 .

Jerretinglés . . . .... 57 Juriola . . . . . . . 34

Jerret de St. Pere. . . . $\quad 57$ Juriola de cría. . . . . . 34

Jerret mascle . . . . . 57 Juriola voladora . . . . 33

Joell. . . . . . . . 66-67

\section{K}

King-yu .

Latría. . . . . . . . 84 Lexola. . . . . . . . 46

Látigo. . . . . . . 50 Limanda. . . . . . 73

Lexa . . . . . . . 46 


\section{டᄂ}

Págs.

Págs.

Llabió.

62 Llissa galubet.

$66^{\circ}$

Llambrega

60

Llissó .

66

Llampresa marina

95 Llissa llubarrera . . . . 65

Llampresa de mar.

95 Llissa de roquer.

66

Llampresa petita .

95 Llissa vera :

66

Llampresa de riu.

95

Llissara

65-66

Llampuga.

Llíssara llubarrera .

65

Llengua de bacallá. .

70

Llop

37

Llengua de St. Pau

74

Llubarro .

37

Llenguado. .

Lluhina.

37

Llenguado d'arena.

75 Lluerna,

:33-34

Llenqueta

32 Lluerneta

34

Llepissós

27 Lluerna pogona .

33

Lligues cames.

50 Lluert.

88

Llissa .

65 Llunada.

14

Llissa agut .

66

Llus. .

69

Llissa cap pla.

66 -Llússara.

69

\section{M}

Mabre.

54 Milana. . . . . . . . . 21

Madrilla

83 Moll

Maira.

69 Moll cranqué .

Maira d'amplova

87. Moll fangué.

Malarmat

33 Moll reyal

Marmota.

33 Moll roqué.

Manta.

21

Moll ve.

Mare d'Anfós

41 Mollera.

$68-71$

Mariquita.

61 Mollera brótola.

71

Massot.

58

Mollera borda.

72

Mata soldats. 56-57

Mollera moranella

69

Maurolicus .

87 Mollera inglesa

Meleta

81 Mollera pigada.

71

Melva.

Menja muxines

44 Mollera roquera

71

18 Mora . . . . . . 56

Meru, .

35-40 Moranell.

19

Metge.

68 Morruda

52

Mila.

21-A Moxarra.

55 
Muret. . . . . . . 64-93 Mussola . . . . . . . 12

Muret vermell . . . . . . 41 Mussola vera. . . . . . . 12

Murena . . . . . . . 93 Muxina . . . . . . . 10

Murer . . . . . . . . 94 Muxó . . . . . 67-89-90

Musich. . . . . . 24-65

\section{N}

Negret

15-16-49 Neru .

38

Negritu

$15-16-49$

Oblada.

53 Oriola.

34

Orada .

55 Oronyola.

87

\section{$P$}

Pagell:

53 Peix de bassa . . . . . . 81

Pagell dento. . . . . . . 56 Peix espasa . . . . . . 10-49

Pagre . . . . . . . . 54 Peix Porch . . . . . . 79-80

Palayó. . . . . . . . 72 Peix rey. . . . . . . . 69

Palomida. . . . . . 46-49 Peix sense sang. . . . 66-67

Palomina. . . . . . . . 49 Pelaya. . . . . . . . 78

Pamfont . . . . . . . 68 Pelaya bruixa. . . . . . 77

Pamfont vermell . . . . 68 Pelaya guarda.la vida : . . 78

Pampol . . . . . . . 37-66 _ Pelaya miseries. . . . 76-77

Pampol pudent. . . . . . 49 Pelaya rossa. . . . . . . 77

Panegal . . . . . . 36 Pelayí. . . . . . . 74-75

Panegall. . . . . . . . 36 Pelerín. . . . . . . . . 12

Pargo. . . . . . . . 54 Peluda. . . . . . . . 75

Pargotí. . . . . . . 54 Pelut. . . . . . . 34

Patoya. . . . . . 84-85 Pelut d'escata . . . . . 35

Pau. . . . . . . 29 Petarch . . . . . 62

Pedás. . . . . . 78-79 Peona. . . . . . . . 33

Pegas. . . . . . . . 50 Pleuronectes . . . . . . 76

Pega roques. . . . . . 79-80 Pillet de platja. . . . . . 92

Peix bade. . . . . . . 24 Pinta ... . . . . . 52

Peix de brom . . . . . . 49 Pitxell. . . . . . . . . 53 
Pixano. . . . . . 68 Pixota vermella . . . . 50

Pixota. . . . . . . . 68 Polla . . . . . . . . 35

Pixota blanca . . . . 68 Porch marí . . . . . . 15-16

Pixota de carall de Jan. . . 68 Puput. . . . . . . . 78-79

Pixota de llanguet .... 68

\section{Q}

Quelet.

55 Quissona.

$\mathbf{R}$

Rabosa. . . . . . 27-28 Rémol empetxinat. . . . 78

Rafalet. . . . . . 33 Rémora . . . . . . 50

Rahó. . . . . . , . 64 Rémol de riu. , . . . . . 73

Rajada . . . 18-19-20-21 Retx . . . . . . 42

Kajada boca de rosa. . . . 20 Rexet. . . . . . . . . 41

Rajada peluda. . . . . . 20 Rey. . . . . . . . . . 41

Rajads vera. . . . . . 18 Reyet. . . . . . . 41

Rajada vestideta . . . . : 20 Roger. . . . . . . . . 32

Rap . . . . . . . . 30 Rogeret . . . . . . . . 32

Rap futaire . . . . . . 30 Rom. . . . . . . . 78

Rascla. . . . ... 35 Romaguera. . . . . . 19

Rascás . . . . . .. 37 Roncador. . . . . . . . 42

Rata .. . . . . . . 26 Roseti. . . . . . . . 32

Red. . . . . . . . 42 Roquer . .. . . . . 59

Refet . . . . . . . 34 Ruch. . . . . . 30-31-32

Refet6. . . . . . . 34 Ruch d'alga. . . . . . . 30

Regalecus. . ... . . 50 Ruch de roca. . . . . . 30-31

Reig . . . . . . 42 Ruflna. . . . . . 36

Rémol. . . . . . 78-79 Rumiguera. . . . . . 19

Rémol blanch . . . . . 79 Ruqué. . . . . 59-60-61

$S$

Áaboga. . . . . 84-85 Salroig. . . . . . . 10

Salmó. .. . . . . . 88-89 Salte murades . . . . . . 88

Salpa . . : . . . 53 Salteperdisa. . . 26

Salproix. . . . . 11 Sama . . . . . . 616 
Sanremus.

75 Serp de mar. . . . . . 93-94

Sard. .

51-52 Serrá. ,

37-38

Sardina .

84-85

Serra

Sardina alatxa.

84 Serrá imperial.

Sardineta

85

Serrandell. .

$76-77$

Sarg

51

Serreta.

Satx.

61-62 Sirvia.

Seitó

$85-86$

Senyoreta

63-64

Sirviola

46

\section{Ser}

Serp. .

66

Solraig.

11

41

2:3-24-92

Somera

67

Serpeta

24-25

Sonsu .

45-65

Serp de costa

24 Surella

Serp d'en terra.

Serpentí.

24 Surelló

45

88 Surell de penya

Taberner.

Tort vert.

58

Tae roques.

63 Totana.

21

Tauró .

14 Totina.

- 21-A

Temburé.

60-62

Tremuló .

17

Tenca .

82

Tremuloya

17

Tiburó.

13 Trenca vits .

67

Tintorera

14

Trompetè.

65

Titulot.

72

Trotllu.

49

Tomba navíos .

66

Truja .

16

Tort.

58-59-60-61-62

Trujeta

62

Tort blanch.

59

Truyta.

89

Tort massot.

58

Truyta salmonada.

89

Tort negre .

Tort ruqué

61 Tunyina.

44

Ull de perdiu 


\section{$\checkmark$}

Págs.

Págs.

Vaca. . . . . 17-18-37-A Verat . . . . . . 43

Vaca serrá . . . . . . . 37 Verderol . . . . . . . 46

Vaca serrana. . . . . . 37-38 Viuda . . . . . . . . 21

Variada . . . . . . . 51 Vit d'en Gahona. . . . . . 64

\section{$\times$}

Xanguet. . . . . . 32-66-67 Xucladó . . . . . 79-80-95

Xasclet . . . . . . . 66 Xucla d'estiu . . . . . . 56

Xavo . . . . . . . . 48 Xucla guindana . . . . 56

Хора . . . . . . . $\quad .55$ Xucla d’hivern. . . . . . 56

Xorich volador . . . . . 33 Xucla vermella . . . . . 56

Xucla. . . . . . . . 56 Xucla vera . . . . . . . 57

Xucla blanca . . . . . 57 Xuriguer. . . . . . 33

Xucla dit. . . . . . . . 79

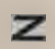

Zanja........ 44

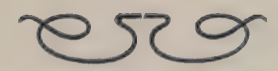








\section{PLEASE DO NOT REMOVE}

CARDS OR SLIPS FROM THIS POCKET

\section{UNIVERSITY OF TORONTO LIBRARY}

QL

618

G52

Biological

\& Medical
Gibert y Oliver, Agustí María Fauna ictiológica de Catalunya 
